\title{
THE CATEGORY THEORETIC SOLUTION OF RECURSIVE PROGRAM SCHEMES
}

\author{
STEFAN MILIUS AND LAWRENCE S. MOSS
}

\begin{abstract}
This paper provides a general account of the notion of recursive program schemes, studying both uninterpreted and interpreted solutions. It can be regarded as the category-theoretic version of the classical area of algebraic semantics. The overall assumptions needed are small indeed: working only in categories with "enough final coalgebras" we show how to formulate, solve, and study recursive program schemes. Our general theory is algebraic and so avoids using ordered, or metric structures. Our work generalizes the previous approaches which do use this extra structure by isolating the key concepts needed to study substitution in infinite trees, including second-order substitution. As special cases of our interpreted solutions we obtain the usual denotational semantics using complete partial orders, and the one using complete metric spaces. Our theory also encompasses implicitly defined objects which are not usually taken to be related to recursive program schemes. For example, the classical Cantor two-thirds set falls out as an interpreted solution (in our sense) of a recursive program scheme.
\end{abstract}

\section{CONTEnTs}

1. Introduction

1.1. Several Semantics

2. Background: Iteratable Functors and Monads

2.1. Iteratable Functors

2.2. Monads

2.3. Recursive Program Schemes 10

2.4. Eilenberg-Moore Algebras 12

3. Completely Iterative Algebras and Complete Elgot algebras 12

3.1. Completely Iterative Algebras $\quad 13$

3.2. Complete Elgot Algebras $\quad 15$

3.3. Computation Tree Elgot Algebras $\quad 17$

3.4. Dramatis Personae 18

3.5. The Eilenberg-Moore Category of $T^{H} \quad 20$

4. Completely Iterative Monads 21

4.1. Second-Order Substitution 24

5. $T^{H}$ as Final Coalgebra among Monads $\quad 25$

5.1. $T^{H}$ Gives a Final Coalgebra as a Monad 25

5.2. $T^{H}$ Gives a Final Coalgebra as a Completely Iterative Monad 29

6. Uninterpreted Recursive Program Schemes 30

7. Interpreted Recursive Program Schemes 36

7.1. Interpreted Solutions in Computation Tree Elgot Algebras $\quad 41$

7.2. Interpreted Solutions in CPO $\quad 43$

7.3. Interpreted Solutions in CMS

8. Conclusions and Future Work

Acknowledgments

Notations

References

Date: 19 March 2007.

Key words and phrases. recursive program scheme, Elgot algebra, coalgebra, completely iterative monad, algebraic trees, second-order substitution . 


\section{INTRODUCTION}

The theory of recursive program schemes (see [C, G,, $\mathrm{N}]$ ) is a topic at the heart of semantics. One takes a system of equations such as

$$
\begin{aligned}
& \varphi(x) \approx F(x, \varphi(G x)) \\
& \psi(x) \approx F(\varphi(G x), G G x)
\end{aligned}
$$

where $F$ and $G$ are given functions and where $\varphi$ and $\psi$ are defined in terms of them by (1.1). The problems are: to give some sort of semantics to schemes, and to say what it means to solve a scheme. Actually, we should distinguish between interpreted schemes, and uninterpreted schemes.

An uninterpreted scheme such as (1.1) above is a purely syntactic construct; one has no information about the data on which given functions and recursively defined ones operate on. Hence, the semantics is independent of this data. In our example the semantics provides two infinite trees

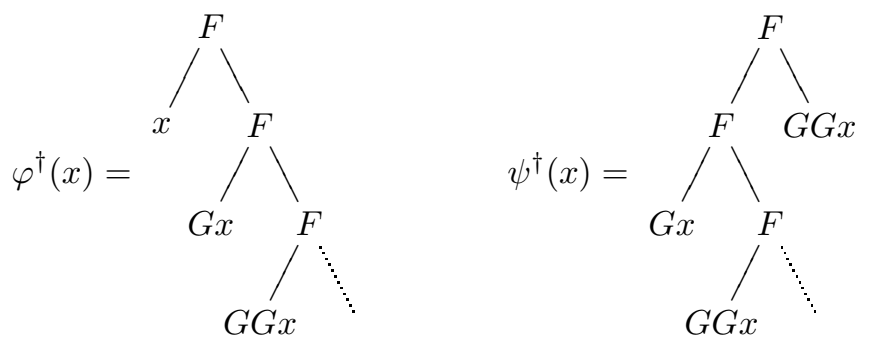

over the signature of given functions; one tree for each of $\varphi$ and $\psi$. We explain this in more detail in Section 1.1 .3 below.

An interpreted scheme is one which comes with an algebra with operations for all the given operation symbols. The recursive program scheme then defines new operations on that algebra. Here is the standard example in the subject. Let $\Sigma$ be the signature of given operation symbols with a constant one, one unary symbol pred, a binary symbol $*$ and a ternary one ifzero. The interpretation we have in mind is the natural numbers where ifzero $\mathbb{N}(k, n, m)$ returns $n$ if $k$ is 0 and $m$ otherwise, and all other operations are as expected. The signature $\Phi$ of the recursively defined operations consists just of one unary symbol $f$. Consider the recursive program scheme

$$
f(n) \approx \operatorname{ifzero}(n, \text { one, } f(\operatorname{pred}(n)) * n)) .
$$

Then (1.3) is a recursive program scheme defining the factorial function.

This paper presents a generalization of the classical theory based on Elgot algebras and final coalgebras. The point in a nutshell is that knowing that the infinite trees for a signature are the final coalgebra for a functor on sets leads to a purely algebraic account of first-order substitution and (co-)recursion, as shown in AAMV, Mo1. One does not need to assume any metric or order to study infinite trees: the finality principle is sufficient for developing the crucial parts of the theory of infinite trees. In this paper we show that this same finality principle allows us to give an uninterpreted semantics to a scheme: we show how to solve a scheme such as (1.1) to get a pair of infinite trees, see (1.2) above.

For our interpreted semantics we work with Elgot algebras, a simple and fundamental notion introduced in $\mathrm{AMV}_{3}$. We show how to give an interpreted solution to recursive program schemes in arbitrary Elgot algebras. We believe that our results in this area generalize and extend the previous work on this topic. Furthermore, we claim that our abstract categorical approach allows a unified view of several well-known approaches to the semantics of implicit recursive function definitions. Our method for obtaining interpreted solutions easily specializes to the usual denotational semantics using complete partial orders. As a second application we show how to solve recursive program schemes in complete metric spaces. For example, it follows from our work that there is a unique contracting $f:[0,1] \longrightarrow[0,1]$ such that

$$
f(x)=\frac{1}{4}\left(x+f\left(\frac{1}{2} \sin x\right)\right) .
$$

In addition, our work on Elgot algebras points to new connections to classical subjects. For example, recall that the Cantor set $c$ is the unique non-empty closed $c \subseteq[0,1]$ such that

$$
c=\frac{1}{3} c \cup\left(\frac{2}{3}+\frac{1}{3} c\right),
$$

where $\frac{1}{3} c$ denotes the set $\left\{\frac{1}{3} x \mid x \in c\right\}$ as usual. The general fact that (1.5) has a unique solution in the set $C([0,1])$ of non-empty closed subsets of $[0,1]$ follows from $C([0,1])$ being an Elgot algebra. 
Finally, our theory also encompasses examples of recursive program schemes and their solutions which are not treated with the classical theory; in this paper we present recursive definitions of operations satisfying equations like commutativity. Other examples are recursive program scheme solutions in non-wellfounded sets (solving $x=\{\{y \mid y \subseteq x$ finite $\}\}$ ). We will present these applications in a future paper $\mathrm{MM}_{3}$.

Our purpose in this paper is to isolate general principles which imply the existence and uniqueness results for uninterpreted solutions of systems such as (1.1) and interpreted schemes such as (1.3 1.5).

1.1. Several Semantics. There are several semantics for recursive program schemes, and it is worth mentioning a bit about them, both to situate our work and also because we shall be interested in showing that one can recover different flavors of semantics from our more general approach.

1.1.1. Operational Semantics. This gives semantics to interpreted schemes only. Solutions are defined by rewriting. In our factorial example, the semantics of (1.3) would be as follows: to compute the solution $f_{\mathbb{N}}(n)$ for a natural number $n$, start with the term $f(n)$, replace $f$ by the right-hand side of the scheme, and then evaluate this term as much as possible. If the evaluated right-hand side still contains one or more symbols $f$, then replace those symbols again by their right-hand side and then evaluate the resulting term, and so on. If after finitely many steps this process returns a natural number, we have computed $f_{\mathbb{N}}(n)$; otherwise we declare $f_{\mathbb{N}}(n)$ to be undefined. (Of course there are important and subtle points to be considered here pertaining to issues like call by name and call by value interpretations of function symbols, and also about the overall strategy of rewriting.) In the factorial example, the described process always stops. But in general this may not be the case.

1.1.2. Denotational Semantics. Again this provides semantics for interpreted schemes only. The algebra that comes as the interpretation of the given functions is a complete partial order (cpo) $A$ with continuous operations for all given operation symbols. A system like (1.1) gives then rise to a continuous function $R$ on a function space. In our factorial example from (1.3), one would consider the natural numbers as a flat cpo and $R$ assigns to a continuous function $f$ on that cpo the function $R f$ defined by

$$
R f(n)=\left\{\begin{array}{cl}
\perp & \text { if } n=\perp \\
1 & \text { if } n=0 \\
f(n-1) \cdot n & \text { else }
\end{array}\right.
$$

The semantics of the given scheme is then the least fixed point of $R$. More generally, denotational semantics provides a continuous operation $\varphi_{A}$ on $A$ for each recursively defined operation symbol $\varphi$ of a given recursive program scheme.

It is true but by no means obvious that the operational and denotational semantics agree in the appropriate sense. Thus, there is a matter of semantic equivalence to be investigated. This was one of the primary starting points of the original theory of recursive program schemes, see $[\mathrm{C}, \mathrm{G}, \mathrm{N}]$. In any case, the equivalence raises a question: the very definition of the denotational semantics seems to require an order. But is an order really necessary? One of the themes of work in coalgebra is that for some semantic problems, order-theoretic methods may be replaced by purely algebraic ones. The reason is that coalgebra is often about semantics spaces for infinite behaviors of one type or another, and these are studied using universal properties (typically finality) instead of the extra structure coming from a cpo or complete metric space. In broad terms, this is our move as well.

1.1.3. Algebraic Semantics. This considers uninterpreted recursive program schemes. These are schemes where no interpretation is given. It is then an issue in this approach to make sure that one can recover the denotational and operational semantics inside the algebraic semantics. But first, one must say what a solution to (1.1) should be. Normally, one considers for a signature $\Sigma$ and a set $X$ of generators the set $T_{\Sigma} X$ of all finite and infinite $\Sigma$-trees over $X$. These are ordered and rooted trees labelled so that an inner node with $n$ children, $n>0$, is labelled by an $n$-ary operation symbol from $\Sigma$, and leaves are labelled by a constant symbol or a variable of $X$. Then one defines an appropriate notion of second-order substitution whereby $\Sigma$-trees may be substituted for operation symbols in $\Gamma$-trees for another signature $\Gamma$ (see [C]). (Recall that the ordinary first-order substitution replaces $\Sigma$-trees for generators in the leaves of other $\Sigma$-trees; only one signature is involved.) In general, a recursive program scheme is given by two signatures: $\Sigma$ (of given operations) and $\Phi$ (of recursively defined operations), and a set of formal equations providing for each $n$-ary operation $\varphi$ from $\Phi$ on the left-hand side a $(\Sigma+\Phi)$-tree over $n$ syntactic variables on the right-hand side of the equation. A solution for such a recursive program scheme then assigns to each $n$-ary operation symbol $\varphi$ from $\Phi$ a $\Sigma$-tree $\varphi^{\dagger}\left(x_{1}, \ldots, x_{n}\right)$ in $n$ syntactic variables. This $\Sigma$-tree is required to be equal to the $\Sigma$-tree obtained by performing the following second-order substitution on the tree from the right-hand side of the 
formal equation defining $\varphi$ : one replaces each operation symbol $\psi$ from $\Phi$ by the $\Sigma$-tree provided by the solution.

As an example, consider the signature $\Sigma$ with a binary operation symbol $F$ and a unary symbol $G$. One might want to define new operations $\varphi$ and $\psi$ recursively as in (1.1) above. Notice that the system is guarded, or in Greibach normal form: the right-hand sides start with a symbol from $\Sigma$. One key opening result of algebraic semantics is that a unique solution exists for guarded systems among $\Sigma$-trees. For instance, for the above system (1.1) the solution consists of the $\Sigma$-trees from (1.2). The standard approach views infinite trees as either the completion of the finite trees, considered as a metric space, or else as the ideal completion of the set of finite trees. Second-order substitution is defined and studied using one of those methods, and using this one can say what it means to solve a recursive program scheme. We shall rework the standard theory by considering $\Sigma$-trees as final coalgebras. More precisely, let $H_{\Sigma}$ be the functor on sets associated to $\Sigma$; see Example 2.1 below. Then the collection $T_{\Sigma} X$ of all $\Sigma$-trees over $X$ is the carrier of a final coalgebra structure for the functor $H_{\Sigma}\left({ }_{-}\right)+X$. It is the universal property of those final coalgebras for any set $X$ which allows us to give a semantics to recursive program schemes. We feel that this move leads to a nicer treatment on a conceptual level.

For example, the solution to (1.1) is obtained from finality alone, as follows: the original recursive program scheme gives rise to a flat system of equations. In this case, this means a function of the form $e: Z \longrightarrow$ $H_{\Sigma} Z+X$, where $Z$ is a set called the set of formal variables of the system and $X$ is the set of syntactic variables from the recursive program scheme. In this particular case, we have $X=\{x\}$, and the set $Z$ of formal variables is the set $T_{\Sigma+\Phi}\{x\}$ of all finite and infinite $(\Sigma+\Phi)$-trees in one variable $x$. Since the formal variables are trees, and since trees are involved with the intended solution, there is much opportunity for confusion. To minimize it, we shall underline the formal variables.

Again, we have a formal variable $\underline{t}$ for each $(\Sigma+\Phi)$-tree $t$. The system $e$ itself works as follows: if $t$ is the variable $x$ we have $e(\underline{x})=x$; we usually prefer to write this as $\underline{x} \approx x$. If $t$ is not $x$, then $e(\underline{t})$ is the result of replacing all appearances of symbols of $\Phi$ by their right-hand sides in the given scheme.

In more technical terms, one performs on $t$ the second-order substitution which is given by the original recursive program scheme (read as an assignment from left to right). This second-order subtitution provides a map $\left({ }_{-}\right)^{\star}: T_{\Sigma+\Phi}\{x\} \longrightarrow T_{\Sigma+\Phi}\{x\}$ satisfying the following equations:

$$
\begin{array}{rlrl}
F(t, u)^{\star} & =F\left(t^{\star}, u^{\star}\right) & G(t)^{\star}=G\left(t^{\star}\right) & x^{\star}=x \\
\varphi(t)^{\star} & =F\left(t^{\star}, \varphi\left(G\left(t^{\star}\right)\right)\right) & \psi(t)^{\star}=F\left(\varphi\left(G\left(t^{\star}\right)\right), G\left(G\left(t^{\star}\right)\right)\right)
\end{array}
$$

In order to arrive in the codomain of $e$ one underlines the maximum proper subtrees of $t^{\star}$. So from (1.1) we obtain the following flat system of equations

$$
\begin{aligned}
& \underline{x} \approx x \quad \underline{\varphi(G x)} \approx F(\underline{G x}, \underline{\varphi(G G x))}
\end{aligned}
$$

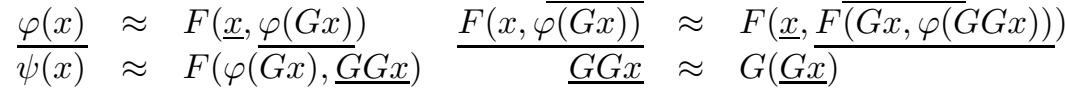

$$
\begin{aligned}
& \underline{\varphi(\overline{\psi(x))}} \approx F(\overline{F(\varphi(G x), G G x), \varphi(G(F(\varphi(G x), G G x))))}
\end{aligned}
$$

and so on. Notice that each tree (here written as a term) on the right-hand side is a $\Sigma$-tree which is either just a syntactic variable or flat; i. e., one operation symbol from $\Sigma$ applied to formal variables. Notice also that the formation of $e$ as described above relies on the fact that the original recursive program scheme is guarded. Now $e$ is, of course, a coalgebra for $H_{\Sigma}($ - $)+X$, and it is well-known that the unique homomorphism from $Z$ to the final coalgebra $T_{\Sigma} X$ assigns to every formal variable its tree unfolding. Hence, this homomorphism assigns to the formal variables $\underline{\varphi(x)}$ and $\underline{\psi(x)}$ their uninterpreted solution in the original recursive program scheme.

At this point, we have explained how we solve recursive program schemes in the algebraic semantics. But much has been omitted. For example, we gave no general account of why ours or any solution method works. Our work to come does provide the missing pieces on this, and much more.

1.1.4. Category Theoretic Semantics. As the title of our paper suggests, our goal is to propose a categorytheoretic semantics of program schemes. The idea is to be even deeper than the algebraic semantics, and to therefore obtain results that are more general. Our theory is based on notions from category theory (monads, Eilenberg-Moore algebras) and coalgebra (finality, solution theorems, Elgot algebras). The overall assumptions are weak: there must be finite coproducts, and all functors we deal with must have "enough final coalgebras". More precisely, we work in a category $\mathcal{A}$ with finite coproducts and with functors $H: \mathcal{A} \longrightarrow \mathcal{A}$ such that for all objects $X$ a final coalgebra $T X$ for $H\left({ }_{-}\right)+X$ exists. We shall introduce and study 
recursive program schemes in this setting. In particular, we are able to prove the existence and uniqueness of interpreted and uninterpreted solutions to schemes. The price we pay for working in such a general setting is that our theory takes somewhat more effort to build. But this is not excessive, and perhaps our categorical proofs reveal more conceptual clarity than the classical ones.

We shall interpret schemes in Elgot algebras. The formal definition of Elgot algebras appears in Section 3 To be brief, they are algebras for a functor $H$ together with an operation which assigns 'solutions' to 'flat equations in $A$ '. This operation must satisfy two easy and well-motivated axioms. One of the key examples of an Elgot algebra is the algebra $T_{\Sigma} X$ of all $\Sigma$-trees over $X$; and indeed, $T_{\Sigma} X$ is a free Elgot algebra on $X$. This fact implies all of the structural properties that are of interest when studying recursion. But in addition, there are many other interesting examples of Elgot algebras. As it happens, continuous algebras are Elgot algebras. We shall show that our general results on solving recursive program schemes in Elgot algebras directly specialize to least fixed-point recursion in continuous algebras. This yields the usual application of recursive program scheme solutions for the semantics of functional programs such as (1.3). Furthermore, algebras on complete metric spaces with contracting operations are Elgot algebras, and so our results specialize to the unique fixed-point recursion in completely metrized algebras provided by Banach's fixed-point theorem. This yields applications such as the ones in (1.4) and (1.5) above.

Related Work. The classical theory of recursive program schemes is compactly presented by Guessarian [G]. There one finds results on uninterpreted solutions of program schemes and interpreted ones in continuous algebras.

The first categorical accounts of infinite trees as monads of final coalgebras appear independently at almost at the same time in work of the second author $\mathrm{Mo}_{1}$, in Ghani et al. GLMP 1 , GLMP 2 , and in Aczel et al. [AAV]. Furthermore, in $\mathrm{Mo}_{1}$ and in $\mathrm{AAV}$, $\mathrm{AAMV}$ it is shown how solutions of formal recursive equations can be studied with coalgebraic methods. In AAMV Mi $i_{1}$ the monad of final coalgebras is characterized as the free completely iterative monad, generalizing and extending results of Elgot et al. E, EBT. Hence, from $\mathrm{AAMV}, \mathrm{Mi}_{1}$ it also follows how to generalize second-order substitution of infinite trees (see Courcelle [C]) to final coalgebras. The types of recursive equations studied in Mon, AAMV] did not go as far as program schemes. It is thus an important test problem for coalgebra to see if work on solving systems of equations can extend to (un)interpreted recursive program schemes. We are pleased that this paper reports a success in this matter.

Ghani et al. GLM] obtained a general solution theorem with the aim of providing a categorical treatment of uninterpreted program scheme solutions. Part of our proof for the solution theorem for uninterpreted schemes is essentially the same as their proof of the same fact. However, the notion of recursive program scheme in GLM is different from ours, and more importantly, our presentation of recursive program scheme solutions as fixed points with respect to (generalized) second-order substitution as presented in [AMV] is new here.

Complete metric spaces as a basis for the semantics of recursive program schemes have been studied by authors such as Arnold and Nivat [AN]. Bloom Bl] studied interpreted solutions of recursive program schemes in so-called contraction theories. The semantics of recursively defined data types as fixed points of functors on the category of complete metric spaces has been investigated by Adámek and Reitermann ARe and by America and Rutten $\mathrm{ARu}$. We build on this with our treatment of self-similar objects. These have also recently been studied in a categorical framework by Leinster, see $\mathrm{L}_{1}, \mathrm{~L}_{2}, \mathrm{~L}_{3}$. The examples in this paper use standard results on complete metric spaces, see, e.g. B. We are not aware of any work on recursive program schemes that even mentions connections to examples like self-similar sets in mathematics, let alone develops the connection.

Finally, some of the results of this paper have appeared in our extended abstract [MM]. However, most of the technical details and all of the proofs were omitted. This full version explains our theory in much more detail and provides full proofs of all new results.

Contents. The paper is structured as follows: Section 2 contains a brief summary of the definitions concerning monads. It also states the overall assumptions that we make in the rest of the paper. Section 3 presents the notions of completely iterative algebra and Elgot algebra, following $\mathrm{AMV}_{3}$. Except for the Section 3.3 none of the results in this section is new. But the paper as a whole would not make much sense to someone unfamiliar with completely iterative algebras and Elgot algebras. So we have included sketches of proofs in this section. The completely iterative monads of Section 4 are also not original. The properties of them developed in Section 5 are needed for the work on uninterpreted schemes (Section 6) and then interpreted schemes (Section 7). These are the heart of the paper. For the convenience of the reader we have included a list of notations and an index at the end. 


\section{Background: Iteratable Functors And Monads}

This section contains most of the background which we need and also connects that background with recursive program schemes. Before reviewing monads in Section 2.2 we should mention the main base categories of interest in this paper, and our overall assumptions on those categories and endofunctors on them.

2.1. Iteratable Functors. Throughout this paper we assume that a category $\mathcal{A}$ with finite coproducts (having monomorphic injections) is given. In addition, all endofunctors $H$ on $\mathcal{A}$ we consider are assumed to be iteratable [sic]: for each object $X$, the functor $H\left({ }_{-}\right)+X$ has a final coalgebra. We denote for an iteratable endofunctor $H$ on $\mathcal{A}$ by

$$
T^{H} X
$$

the final coalgebra for $H\left({ }_{-}\right)+X$. We shall later see that $T^{H}$ is the object assignment of a monad. Whenever confusion is unlikely we will drop the parenthetical $(H)$ and simply write $T$ for $T^{H}$. By the Lambek Lemma $[\mathrm{L}$, the structure morphism

$$
\alpha_{X}^{H}: T^{H} X \longrightarrow H T^{H} X+X
$$

of the final coalgebra is an isomorphism, and consequently, $T^{H} X$ is a coproduct of $H T^{H} X$ and $X$ with injections

$$
\begin{array}{lll}
\eta_{X}^{H} & : X \longrightarrow T^{H} X & \text { "injection of variables" } \\
\tau_{X}^{H} & : H T^{H} X \longrightarrow T^{H} X & \text { "T }
\end{array}
$$

Again, the superscripts will be dropped if confusion is unlikely.

Having coproduct injections that are monomorphic is a mere technicality and could even be totally avoided, see $\mathrm{Mi}_{2}$, Sections 4 and 5. Dropping that assumption would mean replacing the notions of ideal monads and their homomorphisms in Section 4 by two slightly more involved notions. Therefore we decided to keep the additional assumption to simplify our presentation.

More serious is the assumption of iteratability. This is a mild assumption: experience shows most endofunctors of interest are iteratable.

Example 2.1. We recall that ordinary signatures of function symbols $\Sigma$ (as in general algebra) give functors on Set. This is one of the central examples in this paper because it will allow us to recover the classical algebraic semantics from our category-theoretic one. A signature may be regarded as a functor $\Sigma: \mathbb{N} \longrightarrow$ Set, where $\mathbb{N}$ is the discrete category with natural numbers as objects. For each $n$, write $\Sigma_{n}$ for $\Sigma(n)$; this is the set of function symbols of arity $n$ in $\Sigma$. There is no requirement that different numbers should give disjoint sets of function symbols of those arities. Given any signature $\Sigma$ there is an associated polynomial endofunctor (henceforth called a signature functor)

$$
H_{\Sigma} X=\coprod_{i<\omega} \Sigma_{i} \times X^{i}
$$

on Set. When we need to refer to elements of $H_{\Sigma} X$, we shall use the notation $(f, \vec{x})$ for a generic element of $H_{\Sigma} X ; n$ is understood in this notation, $f \in \Sigma_{n}$, and $\vec{x}=\left(x_{1}, \ldots, x_{n}\right)$ is an $n$-tuple of elements of $X$. Also observe that on morphisms $k: X \longrightarrow Y$ the action of $H_{\Sigma}$ is given by $H_{\Sigma} k(f, \vec{x})=(f, \overrightarrow{k x})$, where we write $\overrightarrow{k x}$ for $\left(k x_{1}, \ldots, k x_{n}\right)$. Notice that if $f$ is a constant symbol from $\Sigma_{0}$, then $\vec{x}$ and $\overrightarrow{k x}$ are the unique empty tuple.

Signature functors $H_{\Sigma}$ of Set are iteratable. In fact, consider the set

$$
T_{\Sigma} X
$$

of finite and infinite $\Sigma$-labelled trees with variables from the set $X$. More precisely, $T_{\Sigma} X$ consists of ordered and rooted trees labelled so that a node with $n$ children, $n>0$, is labelled by an operation symbol from $\Sigma_{n}$, and leaves are labelled by constant symbols or variables (elements of $X+\Sigma_{0}$ ).

This set $T_{\Sigma} X$ is the carrier of a final coalgebra for $H_{\Sigma}\left({ }_{-}\right)+X$. The coalgebra structure is the inverse of the pairing of the maps $\tau_{X}: H_{\Sigma} T_{\Sigma} X \longrightarrow T_{\Sigma} X$ and $\eta_{X}: X \longrightarrow T_{\Sigma} X$. The map $\tau_{X}:(f, \vec{t}) \longmapsto f(\vec{t})$ performs "tree tupling": it takes an $n$-ary operation symbol $f$ from $\Sigma$ and an $n$-tuple $\vec{t}$ of $\Sigma$-trees and returns the $\Sigma$-tree obtained by joining all the trees from $\vec{t}$ with a common root node labelled by $f$. And $\eta_{X}$ is the obvious injection which regards each variable as a one-point tree.

So at this point we have seen signature functors on Set as examples of iteratable functors. Unfortunately, we must admit that iteratability is not a very nice notion with respect to closure properties of functors-for example, iteratable functors need not be closed under coproducts or composition. But the main examples 
of base categories $\mathcal{A}$ in this paper are Set, CPO and CMS. In these categories there are stronger yet much nicer conditions that ensure iteratability.

Example 2.2. Accessible endofunctors of Set. Let $\lambda$ be a regular cardinal. An endofunctor $H$ of the category Set of sets and functions is called $\lambda$-accessible it it preserves $\lambda$-filtered colimits. It is shown in AP, Proposition 5.2, that $\lambda$-accessible functors are precisely those endofunctors where for each set $X$ any element $x \in H X$ lies in the image of $H m: H Y \longrightarrow H X$ for some subset $Y \longleftrightarrow X$ of cardinality less than $\lambda$. As usual, we call an endofunctor $H$ finitary if it is $\omega$-accessible and we call $H$ accessible if it is $\lambda$-accessible for some regular cardinal $\lambda$. Any accessible endofunctor is iteratable, see $\mathrm{Ba}$. In particular, signature functors are finitary, whence iteratable (as we already know).

Example 2.3. Locally continuous endofunctors. CPO is the category of $\omega$-complete partial orders (partially ordered sets with joins of all increasing $\omega$-chains, but not necessarily with a least element $\perp$ ). Morphisms of CPO are the continuous functions (preserving joins of all $\omega$-chains). Hence the morphisms are monotone (preserve the order). Notice that coproducts in CPO are disjoint unions with elements of different summands incomparable. CPO is understood to be enriched in the obvious way. For given cpo's $X$ and $Y$, the hom-set $\mathrm{CPO}(X, Y)$ is a cpo in the pointwise order. An endofunctor $H$ on CPO is locally continuous if it preserves the extra structure just noted - that is, each derived function $\mathrm{CPO}(X, Y) \longrightarrow \mathrm{CPO}(H X, H Y)$ is continuous. Observe that not all locally continuous functors need be iteratable. For a counterexample consider the endofunctor assigning to a cpo $X$ the powerset of the set of order components of $X$. This is a locally continuous endofunctor but it does not have a final coalgebra. However, any accessible endofunctor $H$ on $\mathrm{CPO}$ has a final coalgebra, see $\mathrm{Ba}$, and moreover, $H$ is iteratable.

Example 2.4. Contracting endofunctors of complete metric spaces. CMS is the category of complete metric spaces with distances measured in the interval $[0,1]$ together with maps $f: X \longrightarrow Y$ such that $d_{Y}(f x, f y) \leq d_{X}(x, y)$ for all $x, y \in X$. These maps are called non-expanding. A stronger condition is that $f$ be $\varepsilon$-contracting: for some $\varepsilon<1$ we have that $d_{Y}(f x, f y) \leq \varepsilon \cdot d_{X}(x, y)$ for $x, y \in X$. Again, CMS is understood to be enriched. For complete metric spaces $\left(X, d_{X}\right)$ and $\left(Y, d_{Y}\right)$ the hom-set $\mathrm{CMS}(X, Y)$ is a complete metric space with the metric given by

$$
d_{X, Y}(f, g)=\sup _{x \in X} d_{Y}(f(x), g(x)) .
$$

Recall that a functor $H$ on CMS is called $\varepsilon$-contracting if there exists a constant $\varepsilon<1$ such that each derived function $\mathrm{CMS}(X, Y) \longrightarrow \mathrm{CMS}(H X, H Y)$ is an $\varepsilon$-contracting map; that is,

$$
d_{H X, H Y}(H f, H g) \leq \varepsilon \cdot d_{X, Y}(f, g)
$$

for all non-expanding maps $f, g: X \longrightarrow Y$. Contracting functors on CMS are iteratable, see [ARe.

Construction 2.5. Let $H$ be an endofunctor of Set. We recall here that a final coalgebra $T$ for $H$ can (if it exists) be constructed as a limit of an (op-)chain. Let us define by transfinite recursion the following chain indexed by all ordinal numbers:

$$
\begin{array}{lrlrl}
\text { Initial step: } & T_{0} & =1, & t_{1,0} \equiv H 1 \stackrel{!}{\longrightarrow} 1, \\
\text { Isolated step: } & T_{\beta+1} & =H T_{\beta}, & t_{\beta+1, \gamma+1} \equiv H T_{\beta} \stackrel{H t_{\beta, \gamma}}{\longrightarrow} H T_{\gamma} \\
\text { Limit step: } & T_{\lambda} & =\lim _{\beta<\lambda} T_{\beta} & & \text { with limit cone } t_{\lambda, \beta}: T_{\lambda} \longrightarrow T_{\beta}, \quad \beta<\lambda,
\end{array}
$$

where the connecting map $t_{\lambda+1, \lambda}$ is uniquely determined by the commutativity of the squares

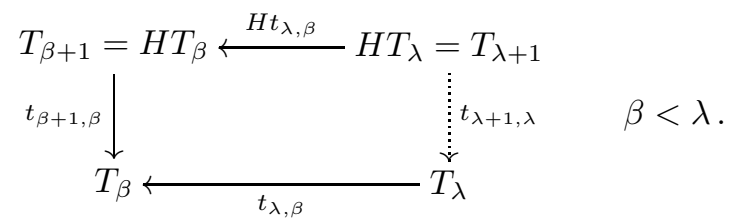

This chain it said to converge if $t_{\beta+1, \beta}$ is an isomorphism for some ordinal number $\beta$.

It has been proved by Adámek and Koubek $\mathrm{AK}$ that an endofunctor $H$ has a final coalgebra iff the above chain converges; moreover, if $\beta$ is an ordinal number such that $t_{\beta+1, \beta}$ is invertible, then $t_{\beta+1, \beta}^{-1}: T_{\beta} \longrightarrow H T_{\beta}$ is a final coalgebra for $H$. For many set endofunctors one can give a bound for the number of steps it will take until the above final coalgebra chain $T_{\beta}$ converges. The following result has been established by Worrell [W]. 
Theorem 2.6. For a $\lambda$-accessible endofunctor $H$ of Set the final coalgebra chain $T_{\beta}$ converges after $\lambda \varnothing 2$ steps, and $\left(T_{\lambda \varnothing 2}, t_{\lambda \varnothing 2+1, \lambda \varnothing 2}^{-1}\right)$ is a final coalgebra for $H$.

In particular, for a finitary endofunctor a final coalgebra is obtained after $\omega+\omega$ steps. For some functors one can further improve on this bound. For endofunctors preserving limits of countable op-chains the final coalgebra chain converges after countably many steps so that $\left(T_{\omega}, t_{\omega+1, \omega}^{-1}\right)$ is a final coalgebra. For example, each signature functor $H_{\Sigma}$ of Set preserves limits of op-chains.

We mention additional examples of iteratable endofunctors $H$ with their final coalgebras $T X$ on the categories of interest.

\section{Examples 2.7.}

(i) A functor $H$ : Set $\longrightarrow$ Set is finitary (it preserves filtered colimits) iff it is a quotient of some polynomial functor $H_{\Sigma}$, see [AT], III.4.3. The latter means that we have a natural transformation $\varepsilon: H_{\Sigma} \longrightarrow H$ with epimorphic components $\varepsilon_{X}$. These components are fully described by their kernel equivalence whose pairs can be presented in the form of so-called basic equations

$$
\sigma\left(x_{1}, \ldots, x_{n}\right)=\rho\left(y_{1}, \ldots, y_{m}\right)
$$

for $\sigma \in \Sigma_{n}, \rho \in \Sigma_{m}$ and $(\sigma, \vec{x}),(\rho, \vec{y}) \in H_{\Sigma} X$ for some set $X$ including all $x_{i}$ and $y_{j}$. Adámek and Milius [AM] have proved that the final coalgebra $T X$ for $H\left({ }_{-}\right)+X$ is given by the quotient $T_{\Sigma} X / \sim_{X}$ where $\sim_{X}$ is the following congruence: for every $\Sigma$-tree $t$ denote by $\partial_{n} t$ the finite tree obtained by cutting $t$ at level $n$ and labelling all leaves at that level by some symbol $\perp$ not from $\Sigma$. Then we have $s \sim_{X} t$ for two $\Sigma$-trees $s$ and $t$ iff for all $n<\omega, \partial_{n} s$ can be obtained from $\partial_{n} t$ by finitely many applications of the basic equations describing the kernel of $\varepsilon_{X}$. For example, the functor $H$ which assigns to a set $X$ the set $\{\{x, y\} \mid x, y \in X\}$ of unordered pairs of $X$ is a quotient of $H_{\Sigma} X=X \times X$ expressing one binary operation $b$ where $\varepsilon_{X}$ is presented by commutativity of $b$; that is, by the basic equation $b(x, y)=b(y, x)$. And $T X$ is the coalgebra of all unordered binary trees with leaves labelled in the set $X$.

(ii) Consider the finite power set functor $\mathcal{P}_{\mathrm{f}}:$ Set $\longrightarrow$ Set. Under the Anti-Foundation Axiom (AFA) A, its final coalgebra is the set $H F_{1}$ of hereditarily finite sets; see [BM]. Analogously, the final coalgebra for $\mathcal{P}_{\mathrm{f}}(\mathrm{-})+X$ is the set $H F_{1}(X)$ of hereditarily finite sets generated from the set $X$. Even without AFA, the final coalgebra for $\mathcal{P}_{\mathrm{f}}$ may be described as in Worrell [W]; it is the coalgebra formed by all strongly extensional trees. These are unordered trees with the property that for every node the subtrees defined by any two different children are not bisimilar. Analogously, the final coalgebra for $\mathcal{P}_{\mathrm{f}}(-)+X$ is the coalgebra of all strongly extensional trees where some leaves have a label from the set $X$.

(iii) The (unbounded) power set functor $\mathcal{P}:$ Set $\longrightarrow$ Set does not have a final coalgebra, whence it is not iteratable. However, moving to the category of classes and functions between them, the power set functor turns out to be iteratable, see e. g. $\mathrm{AMV}_{3}$. Indeed, some of the machinery that comes from iteratable functors turns out to have a surprisingly set-theoretic interpretation when specialized to this setting; see $\mathrm{Mo}_{3}$.

Example 2.8. In our applications, the key point is that certain Set endofunctors lift to (iteratable) endofunctors on CPO. And we need to know that those liftings are locally continuous. In fact, let $H$ be an iteratable Set functor with a locally continuous lifting $H^{\prime}$ on CPO. Thus $H^{\prime}$ is a functor and the forgetful functor $U: \mathrm{CPO} \longrightarrow$ Set gives a commutative square

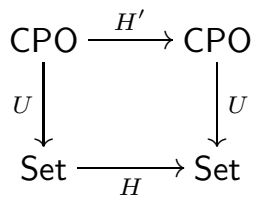

Then $H^{\prime}$ is iteratable, and moreover, the final coalgebra functor $T^{H^{\prime}}$ is a lifting of $T^{H}$ :

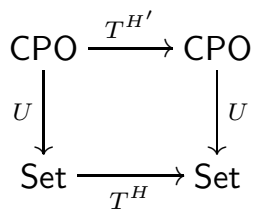


To see this, first recall that for any set $X$ the final coalgebra $T^{H} X$ is obtained from the final coalgebra chain $T_{\beta}$ of $H\left({ }_{-}\right)+X$, see Construction 2.5. In fact, $T^{H} X$ is the coalgebra $\left(T_{\gamma}, t_{\gamma+1, \gamma}^{-1}\right)$ for the smallest ordinal number $\gamma$ for which $t_{\gamma+1, \gamma}$ is invertible. As CPO is a complete category we can define for any endofunctor $H^{\prime}$ of CPO a final coalgebra chain $T_{\beta}^{\prime}$ in precisely the same way as in 2.5. Since the forgetful functor $U$ preserves limits, it follows that for a cpo $X$ the final coalgebra chain of $H^{\prime}(-)+X$ has the $T_{\beta}$ as underlying sets. However, in CPO the continuous map $t_{\gamma+1, \gamma}$ might not be invertible. But since the chain of underlying sets converges at index $\gamma$ we know that for all ordinal numbers $\delta$ the connecting maps $t_{\gamma+\delta, \gamma}: T_{\gamma+\delta} \longrightarrow T_{\gamma}$ are monomorphisms of CPO. Moreover, all cpos $T_{\gamma+\delta}$ have (up to isomorphism) the same underlying set $T_{\gamma}$ and therefore the partial orders on the $T_{\gamma+\delta}, \delta \geq 0$, form a decreasing chain of subsets of $T_{\gamma} \times T_{\gamma}$. This implies that the final coalgebra chain has to converge at some index $\gamma+\delta, \delta \leq \operatorname{card}\left(T_{\gamma} \times T_{\gamma}\right)$. By standard arguments it follows that the cpo $T_{\gamma+\delta}$ is the final coalgebra for $H^{\prime}\left({ }_{-}\right)+X$. Thus, we may choose $T^{H^{\prime}} X=T_{\gamma}$ equipped with the cpo structure given by the cpo $T_{\gamma+\delta}$, whence the square (2.2) commutes as desired.

For example, every signature functor $H_{\Sigma}$ has a locally continuous and iteratable lifting $H^{\prime}$. This lift is the functor

$$
H^{\prime} X=\coprod_{i<\omega} \Sigma_{i} \times X^{i}
$$

on CPO. Here each $\Sigma_{n}$ is a discretely ordered set, + is the coproduct of CPO (a lift of the coproduct of Set), and $\times$ the usual product. It should be noted that even if $X$ has a least element, $H^{\prime} X$ almost never has one. Finally, $T^{H^{\prime}} X$ is the $\Sigma$-tree algebra $T_{\Sigma} X$ with the order induced by the order of the cpo $X$-we describe this order in more detail later in Example 7.14(i).

Example 2.9. Let $H$ : Set $\longrightarrow$ Set have a contracting lifting $H^{\prime}$ on CMS; so we have

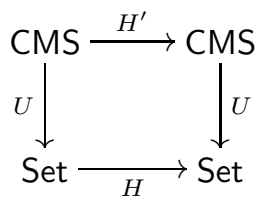

for $U:$ CMS $\longrightarrow$ Set the forgetful functor. Then $H$ is iteratable and $U \varnothing T^{H^{\prime}}=T^{H} \varnothing U$. In fact, this follows from the results of ARe, since $U$ preserves limits. Any signature functor $H$ on Set has a contracting lifting to CMS. For $H X=X^{n}$, define $H^{\prime}(X, d)=\left(X, \frac{1}{2} d_{\max }\right)$ (where $d_{\max }$ is the maximum metric). This is a contracting functor with $\varepsilon=\frac{1}{2}$. And coproducts of $\frac{1}{2}$-contracting liftings are $\frac{1}{2}$-contracting liftings of coproducts. The final coalgebra $T^{H^{\prime}} X$ is the $\Sigma$-tree algebra $T_{\Sigma} X$ equipped with a suitable complete metric. We will provide details of this metric later in Remark 7.16.

Remark 2.10. We comment on the way we formulated the category CPO. Recall from our first mention of it in Example 2.3 that we did not insist that objects in CPO have a least element. This might have seemed mysterious at the time, but now we can explain the reason for this. Requiring a least element and working with strict maps implies that coproducts would not be based on the disjoint union of posets. So the lifting property that we saw in Example 2.8 above would fail. Since this property is important for all of our work with CPO, it dictated the formulation.

2.2. Monads. A monad on a category $\mathcal{A}$ is a triple $(T, \mu, \eta)$ consisting of a functor $T: \mathcal{A} \longrightarrow \mathcal{A}$, and natural transformations $\mu: T T \longrightarrow T$ and $\eta: I d \longrightarrow T$, satisfying the unit laws $\mu \varnothing T \eta=\mu \varnothing \eta T=i d$, and the associative law $\mu \varnothing T \mu=\mu \varnothing \mu T$ :
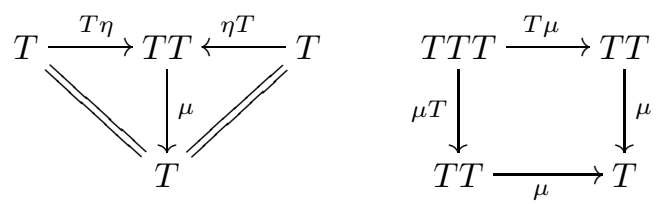

For a functor $H: \mathcal{A} \longrightarrow \mathcal{A}$ and a natural transformation $\alpha: F \longrightarrow G$, we use the usual notations $H \alpha$ and $\alpha H$ to denote the natural transformations with components $H\left(\alpha_{X}\right)$ and $\alpha_{H X}$, respectively. Also it is customary to write just $T$ for the monad in lieu of the triple, and we will follow this convention. 
Let $(S, \eta, \mu)$ and $\left(T, \eta^{\prime}, \mu^{\prime}\right)$ be monads on the same category $\mathcal{A}$. A morphism of monads $\varphi$ from $S$ to $T$ is a natural transformation $\varphi: S \longrightarrow T$ such that $\varphi \varnothing \eta=\eta^{\prime}$, and $\varphi \varnothing \mu=\mu^{\prime} \varnothing(\varphi * \varphi)$ :
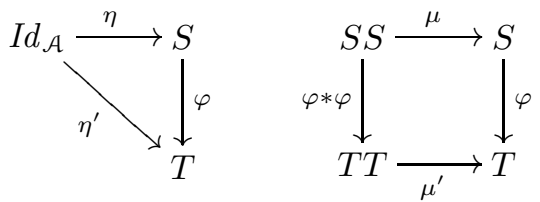

This operation $*$ is the parallel composition of natural transformations. In general, if $\alpha: F \longrightarrow G$ and $\beta: H \longrightarrow K$ are natural transformations, $\alpha * \beta: F H \longrightarrow G K$ is $\alpha K \varnothing F \beta=G \beta \varnothing \alpha H$.

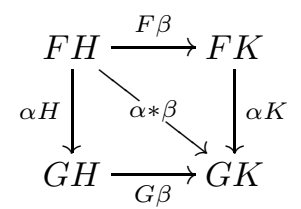

From naturality one easily infers the double interchange law which states that for $\alpha$ and $\beta$ as above and $\alpha^{\prime}: G \longrightarrow G^{\prime}$ and $\beta^{\prime}: K \longrightarrow K^{\prime}$, we have

$$
\left(\alpha^{\prime} * \beta^{\prime}\right) \varnothing(\alpha * \beta)=\left(\alpha^{\prime} \varnothing \alpha\right) *\left(\beta^{\prime} \varnothing \beta\right)
$$

We will denote by

$$
\operatorname{Mon}(\mathcal{A})
$$

the category of monads on $\mathcal{A}$ and their morphisms.

Example 2.11. Let $H_{\Sigma}$ be a signature functor on Set. We already know how to define an object assignment $T_{\Sigma}$. In fact, $T_{\Sigma}$ is a functor. We also have a natural transformation $\eta: I d \longrightarrow T_{\Sigma}$ which for each set $X$ regards the elements of $X$ as elements of $T_{\Sigma} X$. We additionally define a natural transformation $\mu_{X}: T_{\Sigma}\left(T_{\Sigma} X\right) \longrightarrow$ $T_{\Sigma} X$, the operation which takes trees over trees over $X$ into trees over $X$ in the obvious way. In this way, we have a monad $\left(T_{\Sigma}, \mu, \eta\right)$ on Set.

Example 2.12. More generally, let $H$ be iteratable on a category $\mathcal{A}$. It has been shown in previous work AAMV, $\mathrm{Mi}_{2}$ that the object assignment $T$ (assigning to each object $X$ the final coalgebra for $H($ _ $)+X$ ) gives rise to a monad on $\mathcal{A}$. In fact, in loc. cit. this monad is characterized by a universal property - it is the free completely iterative monad on $H$. We will recall the notion of completely iterative monad and the result characterising $T$ in detail later in Sections 3.4 and 4 below.

2.3. Recursive Program Schemes. We shall now explain how to capture recursive program schemes in a category-theoretic way. In order to do this we use the fact that there is a bijective correspondence between natural transformations from a signature $\Sigma$ and from its signature functor $H_{\Sigma}$. More precisely, every signature $\Sigma$ can be regarded as a functor $\Sigma: \mathbb{N} \longrightarrow$ Set, where $\mathbb{N}$ is the discrete category with natural numbers as objects. Let $J: \mathbb{N} \longrightarrow$ Set be the functor which maps a natural number $n$ to the set $\{0, \ldots, n-1\}$. Then for every endofunctor $G$ of Set there is a bijective correspondence

$$
\frac{\Sigma \longrightarrow G \varnothing J}{H_{\Sigma} \longrightarrow G}
$$

and this bijective correspondence is natural in $\Sigma$ and $G$. It is easy to prove this directly. In fact, for any natural transformation $\alpha: \Sigma \longrightarrow G \varnothing J$ (that is, a family of maps $\alpha_{n}: \Sigma_{n} \longrightarrow G\{0, \ldots, n-1\}$ ), we obtain a natural transformation $\beta: H_{\Sigma} \longrightarrow G$ as follows. The component $\beta_{X}$ maps an element $(f, \vec{x})$ of $H_{\Sigma} X$ to $G s\left(\alpha_{n}(f)\right)$, where we consider the $n$-tuple $\vec{x}$ as a function $s: J n \longrightarrow X$. Conversely, given $\beta: H_{\Sigma} \longrightarrow G$ define $\alpha$ by $\alpha_{n}(f)=\beta_{J n}\left(f, i_{n}\right)$, where $i_{n}$ is the $n$-tuple $(0, \ldots, n-1)$. One may verify that the two constructions yield natural transformations, are inverses, and are natural in $\Sigma$ and $G$.

The above bijective correspondence (2.4) also follows from a well-known adjunction between the category of signatures and the category of endofunctors of Set. For two categories $\mathcal{A}$ and $\mathcal{B}$ we denote by

$$
[\mathcal{A}, \mathcal{B}]
$$

the category of functors from $\mathcal{A}$ to $\mathcal{B}$. Recall that the functor ( $) \varnothing J$ : [Set, Set $] \longrightarrow[\mathbb{N}$, Set] of restriction to $\mathbb{N}$ has a left-adjoint $\operatorname{Lan}_{J}($ _ $)$, i. e. the functor assigning to a signature its left Kan extension along $J$. Since 
$\mathbb{N}$ is a discrete category, the usual coend formula for computing $\operatorname{Lan}_{J}(\Sigma)$, see e.g. $\mathrm{ML}$, Theorem X.4.1, specializes to the coproduct

$$
\operatorname{Lan}_{J}(\Sigma) X=\coprod_{n \in \mathbb{N}} \operatorname{Set}(J n, X) \bullet \Sigma(n),
$$

where $M \bullet Z=\coprod_{m \in M} Z$ denotes the copower of $Z$, which is isomorphic to $Z \times M$. Since Set $(J n, X)$ is isomorphic to $X^{n}$ we see that the above formula is isomorphic to the coproduct in (2.1). This implies that $\operatorname{Lan}_{J}(\Sigma)$ is (naturally isomorphic to) the signature functor $H_{\Sigma}$. Hence, by virtue of the adjunction $\operatorname{Lan}_{J}\left({ }_{-}\right) \dashv($ _ $) \varnothing J$, we obtain (2.4) as desired.

We shall now use the above bijective correspondence (2.4) to express recursive program schemes as natural transformations. Suppose we have a signature $\Sigma$ of given operation symbols. Let $\Phi$ be a signature of new operation symbols. Classically a recursive program scheme (or shortly, $R P S$ ) gives for each operation symbol $f \in \Phi_{n}$ a term $t^{f}$ over $\Sigma+\Phi$ in $n$ variables. We thus have a system of formal equations

$$
f\left(x_{1}, \ldots, x_{n}\right) \approx t^{f}\left(x_{1}, \ldots, x_{n}\right), \quad f \in \Phi_{n}, \quad n \in \mathbb{N} .
$$

Recall (1.1) and (1.3) for concrete examples.

Now observe that the names of the variables in (2.5) do not matter. More precisely, an RPS can be presented as a family of functions

$$
\Phi_{n} \longrightarrow T_{\Sigma+\Phi}\{0, \ldots, n-1\}, \quad \text { with } f \longmapsto t^{f}(0, \ldots, n-1) .
$$

So regarding $\Phi$ as a functor from $\mathbb{N}$ to Set, any RPS as in (2.5) gives rise to a natural transformation

$$
\Phi \longrightarrow T_{\Sigma+\Phi} \varnothing J
$$

The formulation in (2.6) insures that in each equation of an RPS such as (2.5), if the symbol on the left side is $n$-ary, then the only variables that can appear on the right are the $n$ elements of $\{0, \ldots, n-1\}$. Notice as well that our formulation extends the classical notion of RPS. Since we used $T_{\Sigma+\Phi}$ in the codomain of (2.6) we have allowed infinite trees instead of just finite terms on the right-hand sides. And we will further generalize this notion of RPS.

The natural transformation in (2.6) corresponds to a unique natural transformation

$$
H_{\Phi} \longrightarrow T_{\Sigma+\Phi} \text {. }
$$

as explained above. The point is that the formulation in (2.7) is more useful to us than the one in (2.6) because (2.7) involves a natural transformations between endofunctors on one and the same category. Notice that for the signature functors $H_{\Sigma}$ and $H_{\Phi}$ we have $H_{\Sigma+\Phi}=H_{\Sigma}+H_{\Phi}$ and hence $T_{\Sigma+\Phi}=T^{H_{\Sigma}+H_{\Phi}}$. With this in mind, we can rewrite (2.7), and we see that recursive program schemes correspond to natural transformations of the following form:

$$
H_{\Phi} \longrightarrow T^{H_{\Sigma}+H_{\Phi}} .
$$

This explains the work we have done so far.

To summarize: we abstract away from signatures and sets and study the uninterpreted and the interpreted semantics of recursive program schemes considered as natural transformations of the form

$$
V \longrightarrow T^{H+V},
$$

where $H, V$ and $H+V$ are iteratable endofunctors on the category $\mathcal{A}$. Now recall from our discussion in the Introduction that to say what a solution of such a recursive program scheme is we first need to have a notion of (generalized) second-order substitution (see [C] for the classical notion-we explain this notion in Example 4.8). It turns out that the universal property of the free completely iterative monads $T^{H}$, see Theorem 4.6, readily yields this desired generalization. And this is the reason we are interested in monads in this paper.

Example 2.13. We mention explicitly how the two recursive program schemes in (1.1) and (1.3) give rise to natural transformations because we later often come back to these running examples. Let $\Sigma$ be the signature that contains a unary operation symbol $G$ and a binary one $F$ - so we have $\Sigma_{1}=\{G\}, \Sigma_{2}=\{F\}$ and $\Sigma_{n}=\emptyset$ else. The signature $\Phi$ of recursively defined operations consists of two unary symbols $\varphi$ and $\psi$. Consider the recursive program scheme (1.1) as a natural transformation $r: \Phi \longrightarrow T_{\Sigma+\Phi} \emptyset J$ with the components given by

$$
r_{1}: \varphi \longmapsto F(0, \varphi(G 0)) \quad \psi \longmapsto F(\varphi(G 0), G G 0)
$$

(we write trees as terms above) and where $r_{n}, n \neq 1$, is the empty map. The bijective correspondence (2.4) yields a natural transformation $H_{\Phi} \longrightarrow T^{H_{\Sigma}+H_{\Phi}}$, where $H_{\Phi} X=X+X$ expresses the recursively defined operations and where $H_{\Sigma} X=X+(X \times X)$ expresses the givens $F$ and $G$. Similarly, the RPS (1.3) defining 
the factorial function with the signature $\Sigma$ of givens and the signature $\Phi$ containing only the unary operation symbol $f$ gives rise to a natural transformation $H_{\Phi} \longrightarrow T^{H_{\Sigma}+H_{\Phi}}$, where $H_{\Sigma} X=1+X+X \times X+X \times X \times X$ and $H_{\Phi} X=X$.

2.4. Eilenberg-Moore Algebras. Recall that if $(F, G, \eta, \epsilon)$ is an adjunction, we get the associated monad on the domain of $F$ by taking $T=G F, \mu=G \epsilon F$, and $\eta$ from the adjunction. We also need a converse of this result. Given a monad $T$ on $\mathcal{A}$, the Eilenberg-Moore category $\mathcal{A}^{T}$ of $T$ has as objects the (monadic) $T$-algebras: these are morphisms $\alpha: T A \longrightarrow A$ such that the diagrams below commute:
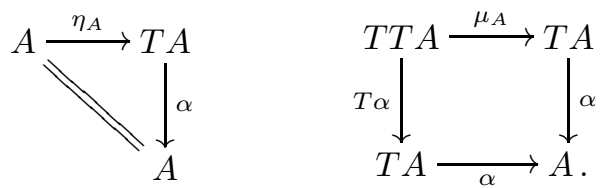

A morphism from $\alpha: T A \longrightarrow A$ to $\beta: T B \longrightarrow B$ is a morphism $h: A \longrightarrow B$ in $\mathcal{A}$ such that the square

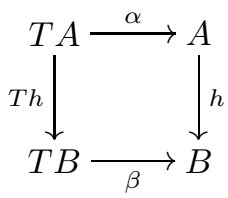

commutes. We sometimes write $T$-algebras using the notation of pairs, as in $(A, \alpha)$, and often simply abbreviate to $A$.

The relation between this construction and monads is that for any monad $T$, there is an adjunction $\left(F^{T}, U^{T}, \eta, \epsilon\right)$ from $\mathcal{A}$ to $\mathcal{A}^{T}$ to which $T$ is associated. Here $F^{T}$ is the functor taking $A$ to the free $T$-algebra $\mu_{A}: T T A \longrightarrow T A ; U^{T}: \mathcal{A}^{T} \longrightarrow \mathcal{A}$ is the forgetful functor taking the $T$-algebra $\alpha: T A \longrightarrow A$ to its carrier $A$; and in the same notation, $\epsilon_{(A, \alpha)}$ is $\alpha$ itself, taken to be a morphism of $T$-algebras, see [ML], Section VI.2.

\section{Completely Iterative Algebras and Complete Elgot algebras}

For interpreted solutions of recursive program schemes we need a suitable notion of an algebra which can serve as interpretation of the givens. By a "suitable algebra" we mean, of course, one in which recursive program schemes have a solution. For example, for the recursive program scheme in (1.1), we are interested in those $\Sigma$-algebras $A$, where $\Sigma$ is the signature that consists of a binary symbol $F$ and a unary one $G$, in which we can obtain two new operations $\varphi_{A}, \psi_{A}$ on $A$ so that the formal equations of (1.1) become valid identities in $A$. In the classical theory one works with continuous algebras - algebras carried by a cpo such that all operations are continuous maps. Alternatively, one can work with algebras carried by a complete metric space such that all operations are contracting maps. In both of these approaches one imposes extra structure on the algebra in a way that makes it possible to obtain the semantics of a recursive definition as a join (or limit, respectively) of finite approximations.

The two types of algebras mentioned above share a crucial feature that allows for the solution of recursive program schemes: these algebras induce an evaluation of all $\Sigma$-trees. More precisely, we consider a $\Sigma$ algebra $A$ with a canonical map $T_{\Sigma} A \longrightarrow A$, providing for each $\Sigma$-tree over $A$ its evaluation in $A$. It seems to us that in order to be able to obtain solutions of recursive program schemes in a $\Sigma$-algebra, the minimal requirement is the existence of such an evaluation map turning $A$ into an Eilenberg-Moore algebra for the monad $T_{\Sigma}$ (see Example 2.11). More generally, we work here with complete Elgot algebras for an iteratable endofunctor $H$, which turn out to be precisely the Eilenberg-Moore algebras for the monad $T^{H}$, see $\mathrm{AMV}_{3}$. An important subclass of all complete Elgot algebras are completely iterative algebras $\mathrm{Mi}_{2}$. One of our main results (Theorem 7.5(ii)) states that recursive program schemes have unique solutions in completely iterative algebras.

We have already seen in the introduction that a recursive program scheme gives rise to a flat system of equations (see (1.7)). So in order to solve recursive program schemes it is sufficient to solve flat systems of equations. Completely iterative algebras are defined as algebras in which every flat system of equations has a unique solution. And an Elgot algebra comes with a choice of a solution for every flat system of equations, and this choice satisfies two easy and natural axioms.

Let us begin by explaining the notion of completely iterative algebra with an example. Let $\Sigma$ be a signature, and let $Y$ be any set. We think of $Y$ as a set of parameters for which we may later substitute $\Sigma$-trees. This is in contrast to the constant symbols from $\Sigma$ which we think of as being fixed once and for all. Consider the $\Sigma$-algebra $T_{\Sigma} Y$ of all (finite and infinite) $\Sigma$-trees over $Y$. Let $X$ be a set (whose elements 
may be considered to be formal variables) which is disjoint from $Y$. A flat system of recursive equations is a systems of formal equations

$$
x \approx t_{x}, \quad \text { for every } x \in X,
$$

where either $t_{x} \in T_{\Sigma} Y$ is a $\Sigma$-tree with no formal variables or else $t_{x}=\sigma\left(x_{1}, \ldots, x_{n}\right), \sigma \in \Sigma_{n}, x_{1}, \ldots, x_{n} \in X$. In this last case, $t_{x}$ is a flat $\Sigma$-tree, a $\Sigma$-tree of height at most 1.

We have already begun in this paper to use the standard practice of using $\approx$ in a system to denote formal equations (recursive specifications of functions or other objects). We use $=$ to denote actual identity (see just below for an example). Flat systems have a unique solution in $T_{\Sigma} Y$ : there exists a unique tuple $x^{\dagger}$, $x \in X$, of trees in $T_{\Sigma} Y$ such that the identities

$$
x^{\dagger}=t_{x}\left[z:=z^{\dagger}\right]_{z \in X}
$$

hold. For example, let $\Sigma$ consist of a binary operation symbol $*$ and a unary one $s$. The following flat system of equations

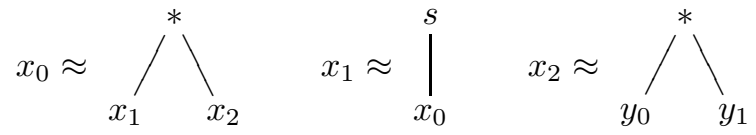

with formal variables $X=\left\{x_{1}, x_{2}, x_{3}\right\}$ and parameters $Y=\left\{y_{0}, y_{1}\right\}$ has as its unique solution the following trees in $T_{\Sigma} Y$ :

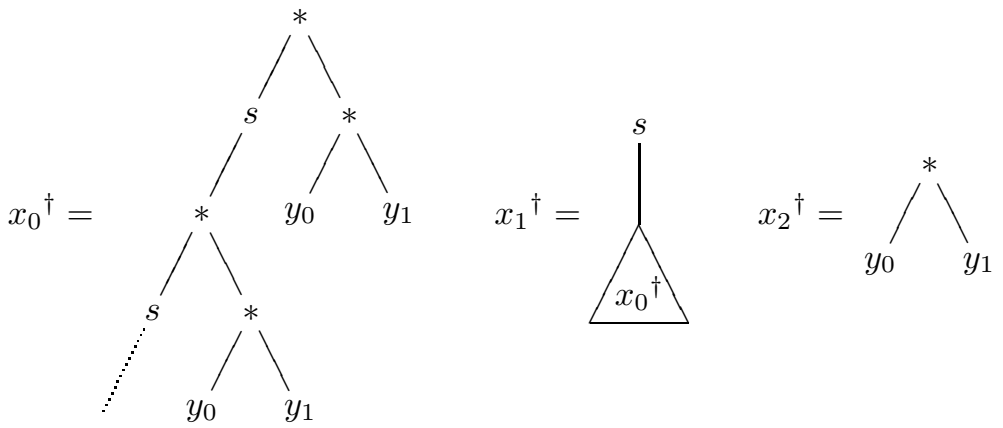

Observe that to give a flat system of equations is the same as to give a mapping $e: X \longrightarrow H_{\Sigma} X+T_{\Sigma} Y$. And a solution is the same as a mapping $e^{\dagger}: X \longrightarrow T_{\Sigma} Y$ such that the following square

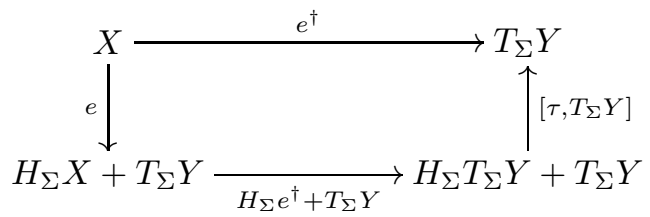

commutes, where $\tau$ denotes the tree-tupling map. (We adopt the convention of denoting in a commutative diagram the identity on an object by that object itself.)

3.1. Completely Iterative Algebras. The example above suggests the following definition, originally studied in $\mathrm{Mi}_{2}$.

Definition 3.1. Let $H: \mathcal{A} \longrightarrow \mathcal{A}$ be an endofunctor. By a flat equation morphism in an object $A$ (of parameters) we mean a morphism

$$
e: X \longrightarrow H X+A \text {. }
$$

Let $a: H A \longrightarrow A$ be an $H$-algebra. We say that $e^{\dagger}: X \longrightarrow A$ is a solution of $e$ in $A$ if the square below commutes:

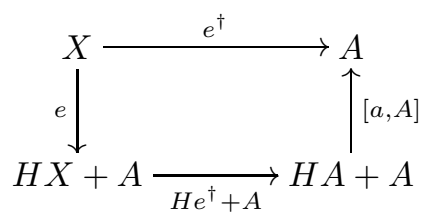

We call $A$ a completely iterative algebra (or cia, for short) if every flat equation morphism in $A$ has a unique solution in $A$. 
Observe that we have no restriction on our objects of variables. (That is, in the case of Set, we do not require that the set of variables be finite.) Imposing this restriction weakens the notion to what $\mathrm{AMV}_{1}$ calls an iterative algebra. It will be essential in this paper to consider equation morphisms whose domain is not finite.

Remark 3.2. We shall see in Section 7 how recursive program schemes as discussed in Section 2.3 can be reduced to flat equation morphisms in an algebra $A$. Then, an interpreted solution of a recursive program scheme in $A$ can be obtained by solving the corresponding flat equation morphism in $A$. In fact, in Theorem 7.5 we will prove that every guarded recursive program scheme has a unique solution in any completely iterative algebra $A$.

\section{Examples 3.3.}

(i) Let $\mathcal{P}_{\mathrm{f}}$ be the finite power set functor on Set, and assume the Anti-Foundation Axiom. Let $H F_{1}$ be the set of hereditarily finite sets. Let $\tau$ be the inclusion of $\mathcal{P}_{\mathrm{f}}\left(H F_{1}\right)$ into $H F_{1}$. This map $\tau$ turns $H F_{1}$ into a cia with respect to $\mathcal{P}_{\mathrm{f}}$.

(ii) Consider the subalgebra $H F_{1 / 2}$ of sets whose transitive closure is finite, then the complete iterativity is lost. Only finite systems can be solved in this setting. For more on this example and the last, see Section 18.1 of $[\mathrm{BM}$.

(iii) Final coalgebras. In $\mathrm{Mi}_{2}$ it is proved that for a final $H$-coalgebra $\alpha: T \longrightarrow H T$ the inverse $\tau: H T \longrightarrow T$ of the structure map yields a cia for $H$. Analogously, for every object $Y$ of $\mathcal{A}$ a final coalgebra $T Y$ for $H\left({ }_{-}\right)+Y$ yields a cia for $H$, see Theorem 3.14 below. This generalizes the first example and the examples $T_{\Sigma} Y$ of all $\Sigma$-trees over a set $Y$.

(iv) Let $H$ be a contracting endofunctor on the category CMS of complete metric spaces, see Example 2.4 Then any non-empty $H$-algebra $(A, a)$ is completely iterative. In fact, given any flat equation morphism $e: X \longrightarrow H X+A$ in CMS, it is not difficult to prove that the assignment $s \longmapsto$ $a \varnothing(H s+A) \varnothing e$ is a contracting function of $\operatorname{CMS}(X, A)$, see $\mathrm{AMV}_{3}$. Then, by Banach's Fixed Point Theorem, there exists a unique fixed point of that contracting function, viz. a unique solution $e^{\dagger}$ of $e$. Notice that $e^{\dagger}$ is obtained as a limit of a Cauchy sequence. In fact, choose some element $\perp \in A$ and define the Cauchy sequence $\left(e_{n}^{\dagger}\right)_{n \in \mathbb{N}}$ in $\operatorname{CMS}(X, A)$ by recursion as follows: let $e_{0}^{\dagger}=$ const $_{\perp}$, and given $e_{n}^{\dagger}$ define $e_{n+1}^{\dagger}$ by the commutativity of the square

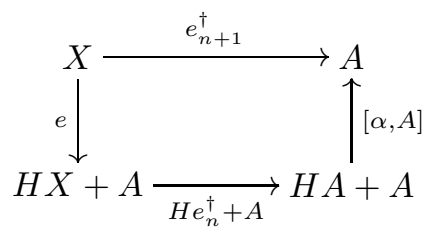

(v) Non-empty compact subsets form cias. Let $(X, d)$ be a complete metric space. Consider the set $C(X)$ of all non-empty compact subspaces of $X$ together with the so-called Hausdorff metric $h$; for two compact subsets $A$ and $B$ of $X$ the distance $h(A, B)$ is the smallest number $r$ such that $B$ can be covered by open balls of radius $r$ around each point of $A$, and vice versa, $A$ can be covered by such open balls around each point of $B$. In symbols, $h(A, B)=\max \{d(A \rightarrow B), d(B \rightarrow A)\}$, where $d(A \rightarrow B)=\max _{a \in A} \min _{b \in B} d(a, b)$. It is well-known that $(C(X), h)$ forms a complete metric space; see, e.g. B]. Furthermore, if $f_{i}: X \longrightarrow X, i=1, \ldots, n$, are contractions of the space $X$ with contraction factors $c_{i}, i=1, \ldots, n$, then it is easy to show that the map

$$
\alpha_{X}: C(X)^{n} \longrightarrow C(X) \quad\left(A_{i}\right)_{i=1, \ldots, n} \longmapsto \bigcup_{i=1}^{n} f_{i}\left[A_{i}\right]
$$

is a contraction with contraction factor $c=\max _{i} c_{i}$ (the product $C(X)^{n}$ is, of course, equipped with the maximum metric). In other words, given the $f_{i}$, we obtain on $C(X)$ the structure $\alpha_{X}$ of an $H$-algebra for the contracting endofunctor $H(X, d)=\left(X^{n}, c \cdot d_{\max }\right)$. Thus, if $X$ is not empty and thus has a compact subset, then $\left(C(X), \alpha_{X}\right)$ is a cia for $H$.

As an illustration we show that the Cantor "middle third" set $c$ may be obtained via the cia structure on a certain space. Recall that $c$ is the unique non-empty closed subset of the interval $I=[0,1]$ which satisfies $c=\frac{1}{3} c \cup\left(\frac{2}{3}+\frac{1}{3} c\right)$. (We write $\frac{1}{3} c$ to mean $\left\{\frac{x}{3} \mid x \in c\right\}$, of course.) So let $(X, d)$ be the Euclidean interval $I=[0,1]$ and consider the $\frac{1}{3}$-contracting functions $f(x)=\frac{1}{3} x$ and $g(x)=\frac{1}{3} x+\frac{2}{3}$ on $I$. Then $\alpha_{I}: C(I) \times C(I) \longrightarrow C(I)$ with $\alpha_{I}(A, B)=f[A] \cup g[B]$ gives the structure of a cia on $C(I)$ for the functor $H(X, d)=\left(X \times X, \frac{1}{3} \cdot d_{\max }\right)$, which is a lifting of the 
signature functor $H_{\Sigma} X=X \times X$ of Set expressing one binary operation symbol $\alpha$. Now consider the formal equation

$$
x \approx \alpha(x, x) .
$$

It gives rise to a flat equation morphism $e: 1 \longrightarrow H 1+C(I)$ which maps the element of the trivial one point space 1 to the element of $H 1=1$. The unique solution $e^{\dagger}: 1 \longrightarrow C(I)$ picks a non-empty closed set $c$ satisfying $c=\alpha(c, c)=f[c] \cup g[c]$. Hence $e^{\dagger}$ picks the Cantor set.

(vi) Continuing with our last point, for each non-empty closed $t \in C(I)$, there is a unique $c(t)$ with $c(t)=\alpha_{I}(c(t), t)$. The argument is just as above. But the work we have done does not show that $c$ is a continuous function of $t$. For this, we would have to study a recursive program scheme $\varphi(x) \approx \alpha(\varphi(x), x)$ and solve this in $\left(C(I), \alpha_{I}\right)$ in the appropriate sense. Our work later in the paper does exactly this, and it follows that the solution to $\varphi(x) \approx \alpha(\varphi(x), x)$ in the given algebra is the continuous function $t \longmapsto c(t)$.

(vii) Suppose that $H$ : Set $\longrightarrow$ Set has a lifting to a contracting endofunctor $H^{\prime}$ on CMS, see Example 2.9 . Let $\alpha: H A \longrightarrow A$ be an $H$-algebra. If there exists a complete metric, say $d$, on $A$ such that $\alpha$ is a nonexpanding map $H^{\prime}(A, d) \longrightarrow(A, d)$, then $A$ is a completely iterative algebra: to every equation morphism $e: X \longrightarrow H X+A$ assign the unique solution of $e:\left(X, d_{0}\right) \longrightarrow H^{\prime}\left(X, d_{0}\right)+(A, d)$, where $d_{0}$ is the discrete metric on $X$, the one given by $d\left(x, x^{\prime}\right)=1$ iff $x \neq x^{\prime}$.

3.2. Complete Elgot Algebras. In many settings, one studies a fixed point operation on a structure like a complete partial order. And in such settings, one typically does not have unique fixed points. So completely iterative algebras are not the unifying concept capturing precisely what is needed to solve recursive program schemes. Instead, we shall need a weakening of the notion of a cia.

Remark 3.4. We will need two operations in the statement of Definition 3.5 below. The first operation formalizes the renaming of parameters in a flat equation morphism. More precisely, for a flat equation morphism $e: X \longrightarrow H X+A$ and a morphism $h: A \longrightarrow B$, we define

$$
h \bullet e \equiv X \stackrel{e}{\longrightarrow} H X+A \stackrel{H X+h}{\longrightarrow} H X+B .
$$

The second operation allows us to combine two flat equation morphisms where the parameters of the first are the variables of the second into one "simultaneous" flat equation morphism. More precisely, given two flat equation morphisms $e: X \longrightarrow H X+Y$ and $f: Y \longrightarrow H Y+A$ we define

$$
f \bullet e \equiv X+Y \stackrel{[e, \mathrm{inr}]}{\longrightarrow} H X+Y \stackrel{H X+f}{\longrightarrow} H X+H Y+A \stackrel{\text { can }+A}{\longrightarrow} H(X+Y)+A,
$$

where can is the canonical morphism $[H \mathrm{inl}, H \mathrm{inr}]$, and where inl and inr denote injections of a binary coproduct.

Definition 3.5. A complete Elgot algebra is a triple $\left(A, a,\left({ }_{-}\right)^{\dagger}\right)$, where $(A, a)$ is an $H$-algebra, and $\left({ }_{-}\right)^{\dagger}$ assigns to every flat equation morphism $e$ with parameters in $A$ a solution $e^{\dagger}: X \longrightarrow A$ such that the following two laws hold:

Functoriality. Solutions respect renaming of variables. Let $e$ and $f$ be two flat equation morphisms with parameters in $A$, and let $h$ be a morphism of equations between them, that is, the square

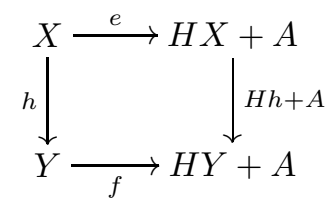

commutes. Then we have

$$
e^{\dagger}=f^{\dagger} \varnothing h .
$$

Compositionality. Simultaneous recursion may be performed sequentially. For all flat equation morphisms $e: X \longrightarrow H X+Y$ and $f: Y \longrightarrow H Y+A$, the solution of the combined equation morphism $f \bullet e$ is obtained by first solving $f$ and then solving $e$ "plugging in" $f^{\dagger}$ for the parameters:

$$
\left(f^{\dagger} \bullet e\right)^{\dagger}=(f \bullet e)^{\dagger} \varnothing \operatorname{inl} .
$$


Definition 3.6. A homomorphism $h$ from an Elgot algebra $\left(A, a,\left({ }_{-}\right)^{\dagger}\right)$ to an Elgot algebra $\left(B, b,\left(_{-}\right)^{\ddagger}\right)$ (for the same functor $H$ ) is a morphism $h: A \longrightarrow B$ that preserves solutions. That is, for every flat equation morphism $e: X \longrightarrow H X+A$ we have a commutative triangle

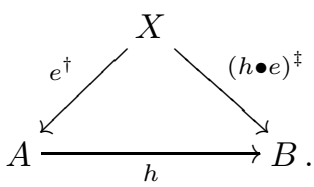

Proposition 3.7. $\mathrm{AMV}_{3}$ Every homomorphism $h:\left(A, a,\left({ }_{-}\right)^{\dagger}\right) \longrightarrow\left(B, b,\left({ }_{-}\right)^{\ddagger}\right)$ of Elgot algebras is a homomorphism of $H$-algebras: the square

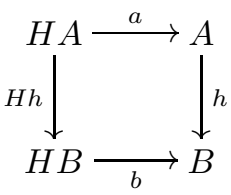

commutes. The converse is false in general. If, however, $A$ and $B$ are cias, then any $H$-algebra morphism is a homomorphism of Elgot algebras.

Sketch of Proof. We show that the square commutes, omitting some of the details. First, consider the flat equation morphism

$$
e_{A} \equiv H A+A \stackrel{H \mathrm{inr}+A}{\longrightarrow} H(H A+A)+A .
$$

Its solution is $e_{A}^{\dagger}=[a, A]: H A+A \longrightarrow A$, as one easily establishes using the commutativity of Diagram 3.2 for $e_{A}^{\dagger}$. Similarly, we have $e_{B}: H B+B \longrightarrow H(H B+B)+B$, and $e_{B}^{\ddagger}=[b, B]$. Now consider $h$ as in our proposition. Then the equation $\left(h \bullet e_{A}\right)^{\ddagger}=h \varnothing e_{A}^{\dagger}$ holds. Furthermore, $H h+h: H A+A \longrightarrow H B+B$ is a morphism of equations from $h \bullet e_{A}$ to $e_{B}$. Thus Functoriality yields $\left(h \bullet e_{A}\right)^{\ddagger}=e_{B}^{\ddagger} \varnothing(H h+h)$. Now one readily performs the computation

$$
[h \varnothing a, h]=h \varnothing e_{A}^{\dagger}=\left(h \bullet e_{A}\right)^{\ddagger}=e_{B}^{\ddagger} \varnothing(H h+h)=[b \varnothing H h, h] .
$$

The desired equation $h \varnothing a=b \varnothing H h$ is the left-hand component of the above equation.

\section{Remark 3.8.}

(i) In $\mathrm{AMV}_{3}$ there is also a notion of a non-complete Elgot algebra. Since we will only be interested in using complete Elgot algebras we will henceforth understand by an Elgot algebra a complete one.

(ii) The axioms of Elgot algebras are inspired by the axioms of iteration theories of Bloom and Ésik [BE]. In fact, the two laws above resemble the functorial dagger implication and the left pairing identity (also known as Bekić-Scott law) from BE].

One justification for the above axioms is that Elgot algebras (for some functor $H$ ) turn out to be the Eilenberg-Moore category of the monad $T^{H}$, see Subsection 3.5. As a result any Elgot algebra $A$ can equivalently be presented by a morphism $T A \longrightarrow A$ satisfying the two usual axioms of Eilenberg-Moore algebras. In particular, for a signature functor $H_{\Sigma}$ on Set we see that if a $\Sigma$-algebra $A$ is an Elgot algebra, then there exists a canonical map $T_{\Sigma} A \longrightarrow A$ which we may think of as an evaluation of all $\Sigma$-trees in $A$.

(iii) Notice that flat equation morphisms are precisely the coalgebras for the functor $H\left({ }_{-}\right)+A$. Thus, the Functoriality above states that $\left({ }_{-}\right)^{\dagger}$ is a functor from the category of coalgebras for $H\left({ }_{-}\right)+A$ to the comma category $\mathcal{A} / A$.

Proposition 3.9. For any endofunctor $H$, every completely iterative algebra is an Elgot algebra.

It is proved in $\mathrm{AMV}_{3}$ that for a cia, the assignment of the unique solution to any flat equation morphism satisfies the Functoriality and the Compositionality laws.

Examples 3.10. We present some further examples of Elgot algebras. None is in general a cia.

(i) Continuous algebras. Let $H$ be a locally continuous endofunctor on CPO, see Example 2.3. It is shown in $\mathrm{AMV}_{3}$ that any $H$-algebra $(A, a)$ with a least element $\perp$ is an Elgot algebra when to a flat equation morphism $e: X \longrightarrow H X+A$ the least solution $e^{\dagger}$ is assigned. More precisely, define $e^{\dagger}$ as the join of the following increasing $\omega$-chain in $\operatorname{CPO}(X, A): e_{0}^{\dagger}$ is the constant function $\perp$; and given $e_{n}^{\dagger}$ let $e_{n+1}^{\dagger}=[a, A] \varnothing\left(H e_{n}^{\dagger}+A\right) \varnothing e$, so that Diagram (3.3) commutes. 
(ii) Suppose that $H$ : Set $\longrightarrow$ Set is a functor with a locally continuous lifting $H^{\prime}: \mathrm{CPO} \longrightarrow \mathrm{CPO}$. An $H$-algebra $\alpha: H A \longrightarrow A$ is called CPO-enrichable if there exists a complete partial order $\sqsubseteq$ on $A$ such that $A$ becomes a continuous algebra $\alpha: H^{\prime}(A, \sqsubseteq) \longrightarrow(A, \sqsubseteq)$ with a least element. Any CPOenrichable algebra $A$ is an Elgot algebra for $H$ : to every flat equation morphism $e: X \longrightarrow H X+A$, let $\leq$ be the discrete order on $X$, consider $\widehat{e}:(X, \leq) \longrightarrow H^{\prime}(X, \leq)+(A, \sqsubseteq)$ defined in the obvious way, and assign $U \hat{e}^{\dagger}: X \longrightarrow A$, where $\widehat{e}^{\dagger}$ is from part (ii), and $U: \mathrm{CPO} \longrightarrow$ Set is the forgetful functor.

(iii) Every complete lattice $A$ is an Elgot algebra for the endofunctor $H X=X \times X$ of Set. In fact, taking binary joins yields an $H$-algebra structure $\vee: A \times A \longrightarrow A$. Furthermore, observe that the algebra $T A$ of all binary trees over $A$ has an evaluation $\alpha: T A \longrightarrow A$ mapping every binary tree in $T A$ to the join of its leaf labels. For any flat equation morphism $e: X \longrightarrow X \times X+A$ form the flat equation morphism $\eta_{A} \bullet e: X \longrightarrow X \times X+T A$. Then take its unique solution $\left(\eta_{A} \bullet e\right)^{\dagger}: X \longrightarrow T A$, and let $e^{*}=\alpha \varnothing\left(\eta_{A} \bullet e\right)^{\dagger}$. One may check that $\left(A, a,\left({ }_{-}\right)^{*}\right)$ is an Elgot algebra for $H$, see $\mathrm{AMV}_{3}$, Example 3.9. Notice that this is usually not a cia since the formal equation $x \approx x \vee x$ has in general many different solutions in a complete lattice.

3.3. Computation Tree Elgot Algebras. In this section we present Elgot algebras for a signature that uses undefined elements and also conditionals. Let $\Sigma$ be a signature, and let $H=H_{\Sigma}$ be the associated endofunctor on Set. Let $(A, a)$ be any $H_{\Sigma^{-}}$-algebra, and let $\uparrow$ be any element of $A$. We shall define an Elgot algebra structure on $A$ related to the natural computation tree semantics of recursive definitions, where solutions are obtained by rewriting. The idea is that $\uparrow$ is our "scapegoat" for ungrounded definitions.

We shall assume that the algebra $A$ interprets the function symbols in $\Sigma$ in a strict fashion: if any argument $a_{i}$ is $\uparrow$, then the overall value $g_{A}\left(a_{1}, \ldots, a_{n}\right)$ is $\uparrow$ as well. Conversely, if $g_{A}\left(a_{1}, \ldots, a_{n}\right)=\uparrow$, we require that ( $n \geq 1$ and) some $a_{i}$ is $\uparrow$. We make this assumption for all function symbols $g$ except for the conditional symbol ifzero $\in \Sigma_{3}$. We make a different assumption on ifzero. For this, fix an element $0 \in A$ other than $\uparrow$. We want

$$
\text { ifzero }_{A}(x, y, z)= \begin{cases}y & \text { if } x=0 \\ z & \text { if } x \neq 0 \text { and } x \neq \uparrow \\ \uparrow & \text { otherwise }\end{cases}
$$

To summarize, in this section we work with algebras for signature functors on Set which come with designated objects $\uparrow$ and 0 satisfying the assumptions above.

We shall work with partial functions and we use some notation which is standard. For partial functions $p, q: X \longrightarrow A, p(x) \uparrow$ means that $p$ is not defined on $x$, and we write $p(x) \downarrow$ if $p$ is defined on $x$. Finally, the Kleene equality $p(x) \simeq q(y)$ means that if either $p(x)$ or $q(y)$ is defined, then so is the other; and in this case, the values are the same.

Definition 3.11. Let $e: X \longrightarrow H X+A$ be a flat equation morphism in $A$. We define a partial function $\widehat{e}: X \longrightarrow A$ as follows:

(i) If $e(x)=a$ and $a \neq \uparrow$, then $\widehat{e}(x) \simeq a$.

(ii) If $e(x)=g\left(x_{1}, \ldots, x_{k}\right), g \neq$ ifzero, and for each $i, \widehat{e}\left(x_{i}\right) \simeq a_{i}$, then $\widehat{e}(x) \simeq g_{A}\left(a_{1}, \ldots, a_{k}\right)$.

(iii) If $e(x)=\operatorname{ifzero}(y, z, w)$ and $\widehat{e}(y) \simeq 0$, then $\widehat{e}(x) \simeq \widehat{e}(z)$.

(iv) If $e(x)=\operatorname{ifzero}(y, z, w)$ and $\widehat{e}(y) \downarrow$ but $\widehat{e}(y) \not \chi 0$, then $\widehat{e}(x) \simeq \widehat{e}(w)$.

We call $\widehat{e}$ the computation function corresponding to $e$.

We intend this to be a definition of a partial function by recursion, so that we may prove facts about $\widehat{e}$ by induction. Here is a first example, one which will be important in Proposition 3.12 below: if $\widehat{e}(x) \downarrow$, then $\widehat{e}(x) \neq \uparrow$.

Now that we have $\widehat{e}$, we define $e^{\dagger}(x)$ to be $\widehat{e}(x)$ if $\widehat{e}$ is defined; if it is not, we set $e^{\dagger}(x)=\uparrow$. (Note that $e^{\dagger}(x)=\uparrow$ iff $\left.\widehat{e}(x) \uparrow.\right)$

In the statement of the result below, we also mention the main way that one obtains structures which satisfy the standing hypotheses of this section.

Proposition 3.12. Let $A_{0}=\left(A_{0}, a_{0}\right)$ be any $H_{\Sigma}$-algebra, let $0 \in A_{0}$, let $\uparrow \notin A_{0}$, and let $A=A_{0} \cup\{\uparrow\}$. Let $A=(A, a)$ be defined in terms of this data by extending $a_{0}$ to the function $a: H_{\Sigma} A \longrightarrow A$ strictly on all function symbols except ifzero, and with ifzero ${ }_{A}$ given by (3.4). Let $\left({ }_{-}\right)^{\dagger}$ be as above. Then $\left(A, a,\left({ }_{-}\right)^{\dagger}\right)$ is an Elgot algebra for $H_{\Sigma}$.

Proof. Clearly the assumption of this section hold for the algebra $A$. These assumptions ensure that $A$ is CPO-enrichable. In fact, equip $A$ with the flat cpo structure with the least element $\uparrow$. Then all operations on 
$A$ are easily checked to be monotone, whence continuous; thus, $a: H_{\Sigma} A \longrightarrow A$ is a continuous algebra. By Example 3.10(ii), we obtain an Elgot algebra $\left(A, a,\left({ }_{-}\right)^{*}\right)$. We will prove that for any flat equation morphism $e: X \longrightarrow H X+A$ the least solution $e^{*}$ agrees with the map $e^{\dagger}$ given by the computation function $\widehat{e}$. To this end recall first that the set $\operatorname{Par}(X, A)$ of partial functions from $X$ to $A$ is a cpo with the order given by $f \sqsubseteq g$ if for all $x \in X, f(x) \downarrow$ implies $g(x) \downarrow$ and $f(x)=g(x)$. Now observe that the definition of $\widehat{e}$ by recursion means that $\widehat{e}$ is the join of an increasing chain in $\operatorname{Par}(X, A)$. In fact, let $\widehat{e}_{0}$ be the everywhere undefined function; and given $\widehat{e}_{n}$ define $\widehat{e}_{n+1}$ as follows: in the clauses (i)-(iv) in Definition 3.11 replace the term $\widehat{e}(x)$ by $\widehat{e}_{n+1}(x)$, and replace all other occurrences of $\widehat{e}$ by $\widehat{e}_{n}$. Then clearly, $\left(\widehat{e}_{n}\right)_{n<\omega}$ is an increasing chain in $\operatorname{Par}(X, A)$ whose join is $\widehat{e}$.

Now recall from Example 3.10(i) that $e^{*}$ is the join of the chain $e_{n}^{*}$ in $\operatorname{CPO}(X, A)$, where $X$ is discretely ordered. We shall show by induction that for every $x \in X$ the equation

$$
e_{n}^{*}(x)= \begin{cases}\widehat{e}_{n}(x) & \text { if } \widehat{e}_{n}(x) \downarrow \\ \uparrow & \text { else }\end{cases}
$$

holds. The base case is obvious. For the induction step we proceed by case analysis. If $e(x)=a$, then $e_{n+1}^{*}(x)=a$ and so (3.5) holds. The second case is $e(x)=g\left(x_{1}, \ldots, x_{k}\right), g \neq$ ifzero. We have $e_{n+1}^{*}(x)=$ $g_{A}\left(e_{n}^{*}\left(x_{1}\right), \ldots, e_{n}^{*}\left(x_{k}\right)\right)$. By our assumptions, this equals $\uparrow$ precisely if at least one of the $e_{n}^{*}\left(x_{j}\right)$ is $\uparrow$, which in turn holds if and only if $\widehat{e}_{n}\left(x_{j}\right) \uparrow$ for some $j$; and equivalently, $\widehat{e}_{n+1}(x) \uparrow$. Otherwise all $\widehat{e}_{n}\left(x_{j}\right)$ are defined and by induction hypothesis we get

$$
e_{n+1}^{*}(x)=g_{A}\left(e_{n}^{*}\left(x_{1}\right), \ldots, e_{n}^{*}\left(x_{k}\right)\right)=g_{A}\left(\widehat{e}_{n}\left(x_{1}\right), \ldots, \widehat{e}_{n}\left(x_{k}\right)\right)=\widehat{e}_{n+1}(x) .
$$

Thirdly, assume that $e(x)=$ ifzero $(y, z, w)$. Then similarly as before we have

$$
e_{n+1}^{*}(x)=\operatorname{ifzero}\left(e_{n}^{*}(y), e_{n}^{*}(z), e_{n}^{*}(w)\right) .
$$

We obtain $e_{n+1}^{*}(x)=\uparrow$ whenever $e_{n}^{*}(y)=\uparrow$. But this happens precisely if $\widehat{e}_{n}(y) \uparrow$, which implies that $\widehat{e}_{n+1}(x) \uparrow$. Now if $e_{n}^{*}(y) \neq \uparrow$, then we have equivalently that $\widehat{e}_{n}(y) \downarrow$. We treat here only the case that $\widehat{e}_{n}(y)=$ 0 ; the remaining case is similar. In our present case it follows that $e_{n+1}^{*}(x)=e_{n}^{*}(z)$ and $\widehat{e}_{n+1}(x) \simeq \widehat{e}_{n}(z)$. Therefore, by the induction hypothesis, the desired equation (3.5) holds.

Finally, from (3.5) we conclude that for the least fixed points $e^{*}$ and $\widehat{e}$ we have

$$
e^{*}(x)= \begin{cases}\widehat{e}(x) & \text { if } \widehat{e}(x) \downarrow \\ \uparrow & \text { else. }\end{cases}
$$

Thus, we get $e^{*}=e^{\dagger}$ which completes the proof.

Definition 3.13. Let $H_{\Sigma}$ : Set $\longrightarrow$ Set be a signature functor, let $A_{0}=\left(A_{0}, a_{0}\right)$ be any $H_{\Sigma}$-algebra as in the hypothesis of Proposition 3.12. We call the Elgot algebra $\left(A, a,\left({ }_{-}\right)^{\dagger}\right)$ the computation tree Elgot algebra induced by $A_{0}$.

We shall study the interpreted solutions of recursive program schemes in computation tree Elgot algebras in Section 7.1 .

3.4. Dramatis Personae. As we have already mentioned, the classical theory of recursive program schemes rests on the fact that for a continuous algebra $A$, there is a canonical map $T_{\Sigma} A \longrightarrow A$. The point is that all $\Sigma$-trees over $A$ can be evaluated in $A$ itself. In a suitable category of cpos the structures $T_{\Sigma} X$ play the rôle of free algebras. The freeness is used to define maps out of those algebras. In our setting, the $\Sigma$-trees are the final coalgebra. So in order to generalize the classical theory, we need a setting in which the final coalgebras $T Y$ for $H\left({ }_{-}\right)+Y$ are free algebras. The following result gives such a setting. It is fundamental for the rest of the paper and collects the results of Theorems 2.8 and 2.10 of $\mathrm{Mi}_{2}$ and Theorem 5.6 of $\mathrm{AMV}_{3}$. We sketch a proof for the convenience of the reader.

Theorem 3.14. Let $H$ be any endofunctor of $\mathcal{A}$. The following are equivalent:

(i) $T Y$ is a final coalgebra for $H\left({ }_{-}\right)+Y$,

(ii) $T Y$ is a free completely iterative algebra for $H$ on $Y$, and

(iii) $T Y$ is a free (complete) Elgot algebra for $H$ on $Y$.

In more detail: if $\left(T Y, \alpha_{Y}\right)$ is a final coalgebra for $H\left({ }_{-}\right)+Y$, the inverse of $\alpha_{Y}: T Y \longrightarrow H T Y+Y$ yields an $H$-algebra structure $\tau_{Y}: H T Y \longrightarrow T Y$ and a morphism $\eta_{Y}: Y \longrightarrow T Y$, which turn $T Y$ into a free cia on $Y$. By Proposition [3.9, $T Y$ is an Elgot algebra for $H$, and additionally this Elgot algebra is also free on $Y$. Conversely, let $\left(T Y, \tau_{Y},\left({ }_{-}\right)^{\dagger}\right)$ be a free Elgot algebra for $H$ with a universal arrow $\eta_{Y}: Y \longrightarrow T Y$. Then $T Y$ is a cia, whence a free cia on $Y$, and $\left[\tau_{Y}, \eta_{Y}\right]$ is an isomorphism whose inverse is the structure map of a final coalgebra for $H\left({ }_{-}\right)+Y$. 
Sketch of Proof. Suppose first that $\left(T Y, \alpha_{Y}\right)$ is a final coalgebra for $H\left({ }_{-}\right)+Y$. Let $\left[\tau_{Y}, \eta_{Y}\right]$ be the inverse of $\alpha_{Y}$. We show that $\tau_{Y}: H T Y \longrightarrow T Y$ is a completely iterative algebra for $H$. In fact, for any flat equation morphism $e: X \longrightarrow H X+T Y$, form the following coalgebra

$$
c \equiv X+T Y \stackrel{[e, \mathrm{inr}]}{\longrightarrow} H X+T Y \stackrel{H X+\alpha_{Y}}{\longrightarrow} H X+H T Y+Y \stackrel{\text { can }+Y}{\longrightarrow} H(X+T Y)+Y
$$

and define

$$
e^{\dagger} \equiv X \stackrel{\text { inl }}{\longrightarrow} X+T Y \stackrel{h}{\longrightarrow} T Y,
$$

where $h$ is the unique homomorphism from the coalgebra $(X+T Y, c)$ to the final one. It is not difficult to prove that $e^{\dagger}$ is the unique solution of $e$.

By Proposition 3.9, it follows that $T Y$ is an Elgot algebra. To establish (ii) and (iii) in the statement of the Theorem it suffices to show that $\left(T Y, \tau_{Y},\left({ }_{-}\right)^{\dagger}\right)$ is a free Elgot algebra on $Y$. For any Elgot algebra $\left(A, a,\left({ }_{-}\right)^{\ddagger}\right)$ and any morphism $m: Y \longrightarrow A$ form the equation morphism

$$
m \bullet \alpha_{Y} \equiv T Y \stackrel{\alpha_{Y}}{\longrightarrow} H T Y+Y \stackrel{H T Y+m}{\longrightarrow} H T Y+A
$$

It is shown in Theorem 5.3 of $\mathrm{AMV}_{3}$ that the solution $h=\left(m \bullet \alpha_{Y}\right)^{\ddagger}$ yields the unique homomorphism $h: T Y \longrightarrow A$ of Elgot algebras such that $h \varnothing \eta_{Y}=m$. In fact, the latter equation follows from the definition of a solution in Diagram (3.2), and the proof that $h$ preserves solutions uses the Compositionality and Functoriality of $\left({ }_{-}\right)^{\ddagger}$. Finally, the uniqueness of $h$ is proved using the Compositionality and Functoriality of $\left({ }^{\prime}\right)^{\dagger}$. The details can be found in loc. cit.

Now conversely, assume that $\left(T Y, \tau_{Y},\left({ }_{-}\right)^{\dagger}\right)$ is a free Elgot algebra for $H$ on $Y$ with a universal arrow $\eta_{Y}: Y \longrightarrow T Y$. It can be shown that $\left[\tau_{Y}, \eta_{Y}\right]$ is an isomorphism, see Lemma 5.7 of $\mathrm{AMV}_{3}$. Denote by $\alpha_{Y}: T Y \longrightarrow H T Y+Y$ its inverse, which is a flat equation morphism. We use $\alpha_{Y}$ to show that $\left(T Y, \tau_{Y}\right)$ is a cia; i. e., for any flat equation morphism $e: X \longrightarrow H X+T Y$ the solution $e^{\dagger}$ is unique. In fact, suppose that $s$ is any solution of $e$. It follows that $s$ is a morphism of equations from $e$ to the flat equation morphism

$$
f \equiv T Y \stackrel{\alpha_{Y}}{\longrightarrow} H T Y+Y \stackrel{H T Y+\eta_{Y}}{\longrightarrow} H T Y+T Y .
$$

Thus, $f^{\dagger} \varnothing s=e^{\dagger}$ by Functoriality of $\left({ }_{-}\right)^{\dagger}$. Next one can show using Compositionality that $f^{\dagger}: T Y \longrightarrow T Y$ is a homomorphism of Elgot algebras satisfying $f^{\dagger} \emptyset \eta_{Y}=\eta_{Y}$. Thus, by the freeness of $T Y, f^{\dagger}=i d$. This proves $s=e^{\dagger}$ so that $\left(T Y, \tau_{Y}\right)$ is a cia, which implies that it is the free one on $Y$. It is not difficult to show that this yields a final coalgebra for $H\left({ }_{-}\right)+Y$. In fact, for any coalgebra $c: C \longrightarrow H C+Y$ the unique solution of the flat equation morphism $\eta_{Y} \bullet c$ yields a unique homomorphism $(C, c) \longrightarrow\left(T Y, \alpha_{Y}\right)$ of coalgebras.

Theorem 3.14 has an important consequence for our work. Recall that we assume $H$ is iteratable, so $H\left({ }_{-}\right)+Y$ does have a final coalgebra for all $Y$. The next result gives the dramatis personae for the rest of the paper.

Theorem 3.15. There is a left adjoint to the forgetful functor from $\operatorname{Alg}^{\dagger} H$, the category of Elgot algebras and their homomorphisms, to the base category $\mathcal{A}$

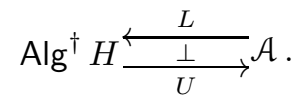

The left-adjoint $L$ assigns to each object $Y$ of $\mathcal{A}$ a free Elgot algebra $\left(T Y, \tau_{Y},\left({ }_{-}\right)^{\dagger}\right)$ on $Y$ with a universal arrow $\eta_{Y}: Y \longrightarrow T Y$ (equivalently, $\left(T Y, \alpha_{Y}\right)$ where $\alpha_{Y}=\left[\tau_{Y}, \eta_{Y}\right]^{-1}$ is a final coalgebra for $\left.H\left({ }_{-}\right)+Y\right)$. The unit of the adjunction is $\eta$ whose components are given by the universal arrows of the free Elgot algebras. The counit $\varepsilon$ gives for each Elgot algebra $\left(A, a,\left(_{-}\right)^{\ddagger}\right)$ the unique homomorphism $\widetilde{a}: T A \longrightarrow A$ of Elgot algebras such that $\widetilde{a} \varnothing \eta_{A}=i d$. We have

$$
\widetilde{a}=\left(\alpha_{A}\right)^{\ddagger}: T A \longrightarrow A,
$$

where $\alpha_{A}: T A \longrightarrow H T A+A$ is considered as a flat equation morphism with parameters in $A$.

Moreover, we obtain additional structure:

(1) A monad $\left(T^{H}, \eta^{H}, \mu^{H}\right)$ on $\mathcal{A}$ such that for all objects $Y$ of $\mathcal{A}$,

(a) $T^{H} Y=T Y$ is the carrier of a final coalgebra for $H\left({ }_{-}\right)+Y$;

(b) $\mu_{Y}^{H}$ is the (unique) solution of $\alpha_{T Y}$, considered as a flat equation morphism with parameters in $T Y$.

(2) A natural transformation $\alpha^{H}: T \longrightarrow H T+I d$. 
(3) A natural transformation $\tau^{H}: H T \longrightarrow T$ such that $\left[\tau^{H}, \eta^{H}\right]$ is the inverse of $\alpha^{H}$.

(4) A "canonical embedding" $\kappa^{H}$ of $H$ into $T: \quad \kappa^{H} \equiv H \stackrel{H \eta^{H}}{\longrightarrow} H T \stackrel{\tau^{H}}{\longrightarrow} T$.

Proof. For every object $Y$, the free Elgot algebra $T Y$ provides a universal arrow $\eta_{Y}: Y \longrightarrow T Y$ from $Y$ to $U$, cf. [ML, III.1. This family of universal arrows for every object $Y$ completely determines the left-adjoint to $U$ (see [ML, Theorem IV.1.2). More concretely, $L$ assigns to a morphism $f: Y \longrightarrow Z$ the unique homomorphism from $T Y$ to $T Z$ extending $\eta_{Z} \varnothing f$. Then functoriality of $L$ and naturality of $\eta$ and $\varepsilon$ as well as the adjunction equations all follow easily from the freeness of the Elgot algebras $T Y$.

The obtained adjunction $L \dashv U$ gives rise to a monad $\left(T^{H}, \eta^{H}, \mu^{H}\right)$ on $\mathcal{A}$ which assigns to every object of $\mathcal{A}$ the underlying object $T Y$ of a free Elgot algebra on $Y$, see Section 2.4. Thus item (1a) follows from Theorem [3.14. The monad multiplication $\mu^{H}$ is given by $U \varepsilon L$, i. e., $\mu_{Y}^{H}: T T Y \longrightarrow T Y$ is the unique homomorphism of Elgot algebras such that $\mu_{Y}^{H} \phi \eta_{T Y}^{H}=i d$. It follows from the proof of Theorem 3.14 that $\widetilde{a}=\left(m \bullet \alpha_{A}\right)^{\ddagger}$, where $m$ is the identity on $A$. The special instance of this where $A$ is the free Elgot algebra $T Y$ yields (1b). From the freeness of the $T Y$ we also infer that the algebra structures $\tau_{Y}$, and the coalgebra structures $\alpha_{Y}$, form natural transformations. Finally, by definition we have that $\alpha^{H}$ and $\left[\tau^{H}, \eta^{H}\right]$ are mutually inverse.

We call the monad $\left(T^{H}, \eta^{H}, \mu^{H}\right)$ the completely iterative monad generated by $H$. (The name comes from an important property which we discuss in Section 4) As always, we just write $T^{H}$, or even $T$ to denote this monad, and we shall frequently drop all superscripts when dealing with the structure coming from a single endofunctor $H$. (But as the reader will see later, we frequently do need to consider the structures coming from two endofunctors. This is particularly pertinent in our study since in recursive program schemes we usually have two signatures, hence two functors, see Section 2.3).

For any Elgot algebra $\left(A, a,\left({ }_{-}\right)^{\dagger}\right)$ for $H$ we call the homomorphism $\widetilde{a}: T A \longrightarrow A$ in (3.6) above the evaluation morphism of that Elgot algebra. Theorem 3.16 below shows that Elgot algebras are equivalently presented by their evaluation morphisms.

\subsection{The Eilenberg-Moore Category of $T^{H}$.}

Theorem 3.16. $\mathrm{AMV}_{3}$ The category $\mathrm{Alg}^{\dagger} \mathrm{H}$ of Elgot algebras is isomorphic to the Eilenberg-Moore category $\mathcal{A}^{T}$ of monadic algebras for the completely iterative monad $T$ generated by $H$. More precisely, for any Elgot algebra $\left(A, a,\left({ }_{-}\right)^{\dagger}\right)$, the evaluation morphism $U \epsilon_{A}=\widetilde{a}: T A \longrightarrow A$ is an Eilenberg-Moore algebra for $T$.

Conversely, for any Eilenberg-Moore algebra $a: T A \longrightarrow A$ we obtain an Elgot algebra by using as structure map the composite $a ø \kappa_{A}: H A \longrightarrow A$, and by defining for a flat equation morphism $e: X \longrightarrow H X+A$ the solution $e^{\dagger}=a \varnothing h$, where $h$ is the unique coalgebra homomorphism from $(X, e)$ to $\left(T A, \alpha_{A}\right)$ :

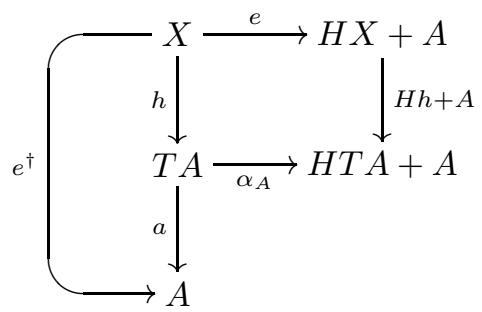

These two constructions extend to the level of morphisms, and they yield the desired isomorphism between the two categories $\operatorname{Alg}^{\dagger} H$ and $\mathcal{A}^{T}$.

Corollary 3.17. The diagrams

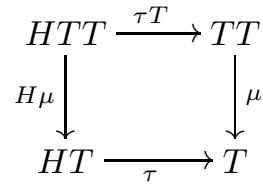

and

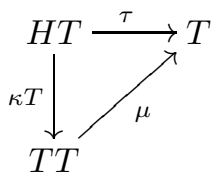

commute, and for every Elgot algebra $\left(A, a,\left({ }_{-}\right)^{\dagger}\right)$ the triangle

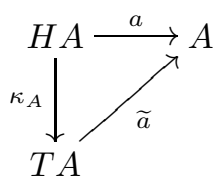


commutes.

Proof. To see that the lower triangle commutes, observe first that $\widetilde{a}$ is a homomorphism of Elgot algebras. So it is an $H$-algebra homomorphism by Proposition 3.7. Now use this fact together with the equations $\kappa_{A}=\tau_{A} \varnothing H \eta_{A}$ (see Theorem 3.15(4)) and $\widetilde{a} \varnothing \eta_{A}=i d$. The special cases of this triangle for $A=T Y$ and $a=\tau_{Y}$ yield the commutativity of the upper right-hand triangle since $\mu_{Y}=U \varepsilon_{T Y}=\widetilde{\tau_{Y}}$. Finally, the upper left-hand square commutes since for each $Y$ in $\mathcal{A}, \mu_{Y}$ is a homomorphism of Elgot algebras, whence an $H$-algebra homomorphism by Proposition 3.7 again.

\section{Completely Iterative Monads}

Before we can state a theorem providing solutions of (generalized) recursive program schemes, we need to explain what a solution is. In the classical setting one introduces second-order substitution of all $\Sigma$-trees. This is substitution of trees for operation symbols, see C. We present a generalization of second-order substitution to the final coalgebras given by $T^{H}$.

In fact, in [AAMV, $\mathrm{Mi}_{2}$ it is proved that the monad $T^{H}$ of Theorem 3.15 is characterized by an important universal property - it is the free completely iterative monad on $H$. This freeness of $T^{H}$ specializes to secondorder substitution of $\Sigma$-trees, a fact we illustrate at the end of the current section.

Here we shall quickly recall those results of [AAMV] which we will need in the current paper. For a well-motivated and more detailed exposition of the material presented here we refer the reader to one of $\mathrm{AAMV}, \mathrm{Mi}_{2}$.

Example 4.1. We have seen in Section 3 that for a signature $\Sigma$, flat systems of formal equations have unique solutions whose components are $\Sigma$-trees over a set of parameters. But it is also possible to uniquely solve certain non-flat systems of equations. More precisely, for a given signature $\Sigma$, consider a system of equations as in (3.1), but where each right-hand side $t_{x}, x \in X$, is any $\Sigma$-tree from $T_{\Sigma}(X+Y)$ which is not just a single variable from $X$. Such systems are called guarded. Guardedness suffices to ensure the existence of a unique solution of the given system.

For example, let $\Sigma$ consist of binary operations + and $*$ and a constant 1 . The following system of equations
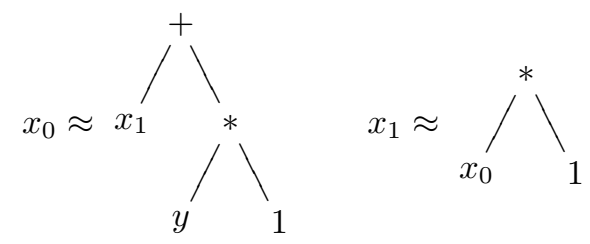

with formal variables $X=\left\{x_{0}, x_{1}\right\}$ and parameters $Y=\{y\}$ is guarded. The solution is given by the following trees in $T_{\Sigma} Y$ :
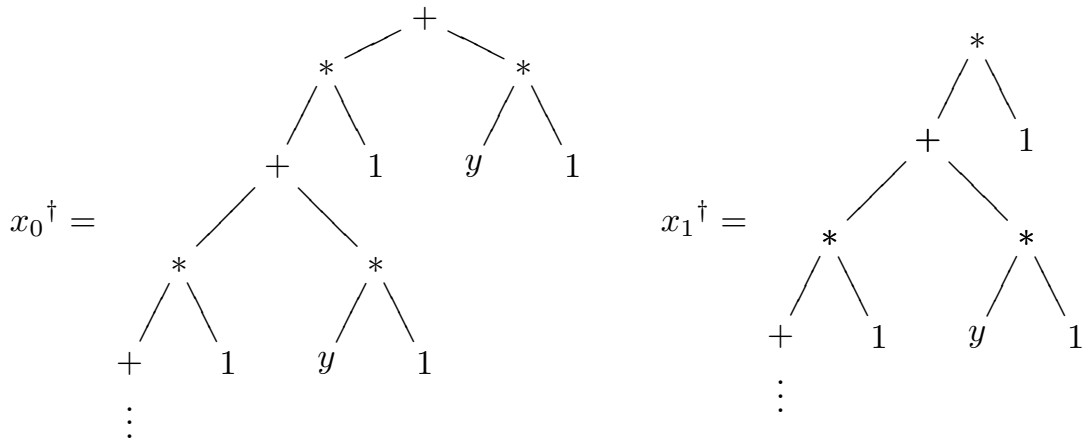

A system of equations as considered in this example is precisely a map $X \longrightarrow T_{\Sigma}(X+Y)$. And a solution is given by a map $e^{\dagger}: X \longrightarrow T_{\Sigma} Y$ such that the square below commutes:

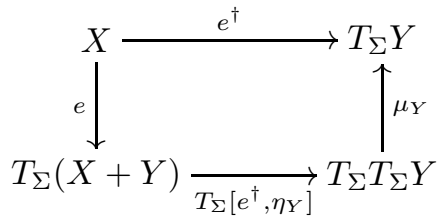


Remark 4.2. In lieu of the monad $T_{\Sigma}$ in Example 4.1 one can more generally consider equation morphisms $X \longrightarrow T(X+Y)$ and their solutions $X \longrightarrow T Y$ for every monad $T=T^{H}$ of Theorem 3.15 above. Notice also that any flat equation morphism $e: X \longrightarrow H X+Y$ gives rise to an equation morphism

$$
X \stackrel{e}{\longrightarrow} H X+Y \stackrel{\kappa_{X}+\eta_{Y}}{\longrightarrow} T X+T Y \stackrel{\text { can }}{\longrightarrow} T(X+Y) .
$$

It is easy to see that solutions of this equation morphism are in one-to-one correspondence with solutions of $\eta_{Y} \bullet e$ in the cia TY (see AAMV], Lemma 3.4).

In [Mo1, AAMV] it was proved that for every monad $T^{H}$ any guarded equation morphisms has a unique solution. Before we state this result precisely we recall the notion of an ideal monad. It adds to an arbitrary monad $S$ enough structure to be able to speak of guarded equations for $S$.

Definition 4.3. By an ideal monad we mean a sextuple

$$
\left(S, \eta, \mu, S^{\prime}, \iota, \mu^{\prime}\right)
$$

consisting of a monad $(S, \eta, \mu)$, and a (right) ideal $\left(S^{\prime}, \mu^{\prime}\right)$, which consists of a subfunctor $\iota: S^{\prime} \hookrightarrow S$, that is a monomorphism $\iota$ in the functor category $[\mathcal{A}, \mathcal{A}]$, and a natural transformation $\mu^{\prime}: S^{\prime} S \longrightarrow S^{\prime}$ such that the following two conditions hold:

(i) $S=S^{\prime}+I d$ with coproduct injections $\iota$ and $\eta$

(ii) $\mu$ restricts to $\mu^{\prime}$ along $\iota$ in the sense that the square below commutes:

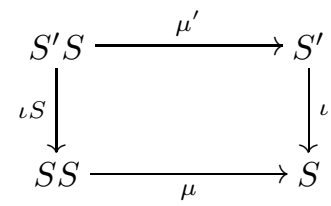

An ideal monad morphism from an ideal monad $\left(S, \eta^{S}, \mu^{S}, S^{\prime}, \iota, \mu^{\prime S}\right)$ to an ideal monad $\left(U, \eta^{U}, \mu^{U}, U^{\prime}, \omega, \mu^{\prime}\right)$ is a monad morphism $m:\left(S, \eta^{S}, \mu^{S}\right) \longrightarrow\left(U, \eta^{U}, \mu^{U}\right)$ which has a domain-codomain restriction to the ideals. More precisely, this means that there exists a natural transformation $m^{\prime}: S^{\prime} \longrightarrow U^{\prime}$ such that the square below commutes:

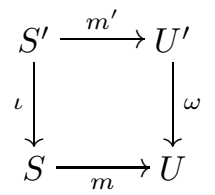

For any endofunctor $H$ and ideal monad $S$, a natural transformation $\sigma: H \longrightarrow S$ is ideal if it factors through the ideal $\iota: S^{\prime} \longleftrightarrow S$ as follows:

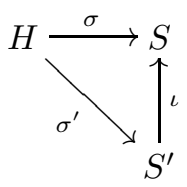

Example 4.4. Recall that the underlying functor of the monad $T$ of Theorem 3.15 is a coproduct of $H T$ and $I d$. Taking for $\iota$ the left-hand coproduct injection $\tau: H T \longleftrightarrow T$ and for $\mu^{\prime}$ the natural transformation $H \mu: H T T \longrightarrow H T$ we see that $T$ is an ideal monad. Furthermore, since $\kappa: H \longrightarrow T$ in Theorem 3.15(4) is $\tau \varnothing H \eta, \kappa$ is an ideal natural transformation.

Definition 4.5. $\quad$ (i) For an ideal monad $S$ on $\mathcal{A}$ an equation morphism is a morphism

$$
e: X \longrightarrow S(X+Y) \text {. }
$$

It is called guarded if it factors as follows:

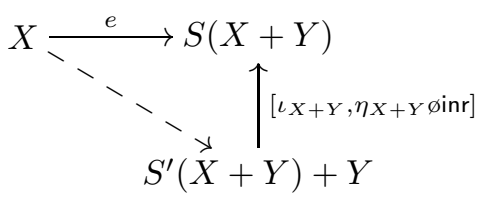


(ii) A solution of an equation morphism $e$ is a morphism $e^{\dagger}: X \longrightarrow S Y$ such that the square below commutes:

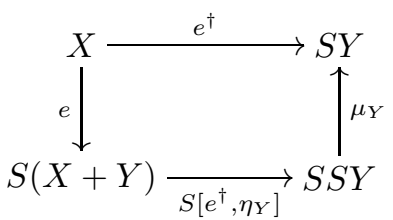

(iii) An ideal monad is called completely iterative provided that any guarded equation morphism has a unique solution.

The first item of the following result is called the Parametric Corecursion Theorem in $\mathrm{Mo}_{1}$ and the Solution Theorem in AAMV]. See also $\mathrm{Mi}_{2}$ for an extension of this result to all cias. The second item is the main result of $\mathrm{AAMV}, \mathrm{Mi}_{2}$.

Theorem 4.6. For any iteratable endofunctor $H$,

(i) the ideal monad $T^{H}$ is completely iterative, and

(ii) $T^{H}$ is free on $H$. More precisely, for all completely iterative monads $S$ and ideal natural transformations $\sigma: H \longrightarrow S$, there exists a unique monad morphism $\bar{\sigma}: T \longrightarrow S$ such that $\bar{\sigma} \cdot \kappa^{H}=\sigma$ :

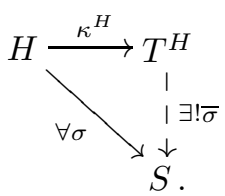

And the induced morphism $\bar{\sigma}$ is an ideal monad morphism.

Sketch of Proof. Let $\left(S, \eta^{S}, \mu^{S}, S^{\prime}, \iota, \mu^{\prime}\right)$ be a completely iterative monad. For every object $Y$ of $\mathcal{A}$ consider $S Y$ as an $H$-algebra with the structure

$$
H S Y \stackrel{\sigma_{S Y}}{\longrightarrow} S S Y \stackrel{\mu_{Y}}{\longrightarrow} S Y .
$$

This is a completely iterative algebra. In fact, every flat equation morphism $e: X \longrightarrow H X+S Y$ yields the following equation morphism w.r.t. the completely iterative monad $S$ :

$$
\bar{e} \equiv X \stackrel{e}{\longrightarrow} H X+S Y \stackrel{\sigma_{X}+S A}{\longrightarrow} S X+S Y \stackrel{\text { can }}{\longrightarrow} S(X+Y) .
$$

It is easy to verify that $\bar{e}$ is guarded, and that solutions of $\bar{e}$ w.r.t. the completely iterative monad $S$ are in 1-1-correspondence with solutions of $e$ in the algebra $S Y$. Thus, since $\bar{e}$ has a unique solution, so does $e$.

Now use that $\left(T Y, \tau_{Y}\right)$ is a free cia on $Y$ to obtain a unique $H$-algebra homomorphism $\bar{\sigma}_{Y}: T Y \longrightarrow S Y$ with $\bar{\sigma}_{Y} \varnothing \eta_{Y}=\eta_{Y}^{S}$. One readily proves that $\bar{\sigma}$ is natural in $Y$, that it is a monad morphism from $T$ to $S$, that it is uniquely determined, and that it is an ideal monad morphism. See Theorem 4.4 of $\left[\mathrm{Mi}_{2}\right.$ for the details.

In our work in the subsequent sections we shall often use the special case of Theorem 4.6 where the completely iterative monad $S$ is $T^{K}$ for some iteratable endofunctor $K$. For that special case we need the following explicit description of the restriction of the monad morphism $\bar{\sigma}$ to the subfunctors $H T^{H}$ and $S^{\prime}=K T^{K}$.

Lemma 4.7. If $H$ and $K$ are iteratable endofunctors and $\sigma: H \longrightarrow T^{K}$ is an ideal transformation, i.e., $\sigma=\tau^{K} \varnothing \sigma^{\prime}$, then for the unique induced ideal monad morphism $\bar{\sigma}$ the following is a commutative diagram:

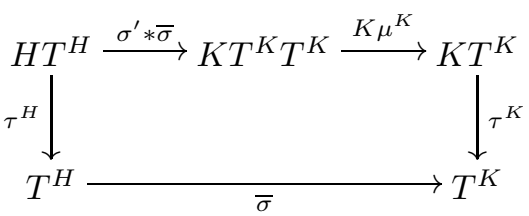

(Recall that $*$ denotes parallel composition of natural transformations.) 
Proof. We verify that the diagram

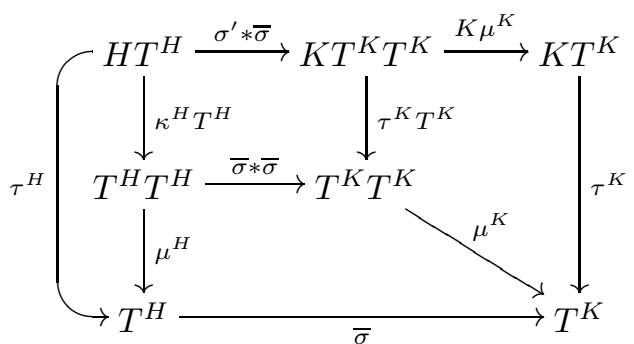

commutes. The left-hand and right-hand parts commute by Corollary 3.17. The upper part commutes by the double interchange law (2.3) together with $\bar{\sigma} \varnothing \kappa^{H}=\sigma=\tau^{K} \varnothing \sigma^{\prime}$, and the lower one since $\bar{\sigma}$ is a monad morphism.

4.1. Second-Order Substitution. For signature functors on Set, the freeness of $T=T^{H_{\Sigma}}$ specializes to second-order substitution - substitution of (finite or infinite) trees for operation symbols. Second-order substitution is a key point in the classical theory of recursive program schemes because the notion of an uninterpreted solution rests on it. We believe that the connection of second-order substitution and any notion of freeness is new.

Example 4.8. Let $\Sigma$ and $\Gamma$ be signatures (considered as functors $\mathbb{N} \longrightarrow$ Set). Each symbol $\sigma \in \Sigma_{n}$ is considered as a flat tree in $n$ variables. A second-order substitution gives an "implementation" to each such $\sigma$ as a $\Gamma$-tree in the same $n$ variables. We model this by a natural transformation $\ell: \Sigma \longrightarrow T_{\Gamma} \varnothing J$, i. e., a family of maps $\ell_{n}: \Sigma_{n} \longrightarrow T_{\Gamma}\{0, \ldots n-1\}, n \in \mathbb{N}$. By the bijective correspondence (2.4) in Section 2.3. this gives rise to a natural transformation $\lambda: H_{\Sigma} \longrightarrow T_{\Gamma}$. When infinite trees are involved there is usually the restriction to so-called non-erasing substitutions, those where $\ell$ assigns to each $\Sigma$-symbol a $\Gamma$-tree which is not just single node tree labelled by a variable. Translated along (2.4) that means precisely that $\lambda$ is an ideal natural transformation. Thus, from Theorem 4.6 we get a monad morphism $\bar{\lambda}: T_{\Sigma} \longrightarrow T_{\Gamma}$. For any set $X$ of variables, its action is that of second-order substitution: $\bar{\lambda}_{X}$ replaces every $\Sigma$-symbol in a tree $t$ from $T_{\Sigma} X$ by its implementation according to $\lambda$. More precisely, let $t$ be a tree from $T_{\Sigma} X$. If $t=x$ is a variable from $X$, then $\bar{\lambda}_{X}(t)=x$. Otherwise we have $t=\sigma\left(t_{1}, \ldots, t_{n}\right)$ with $\sigma \in \Sigma_{n}$ and $t_{i} \in T_{\Sigma} X, i=1, \ldots, n$. Let $\ell_{n}(\sigma)=t^{\prime}(0, \ldots, n-1) \in T_{\Gamma}\{0, \ldots, n-1\}$. Then the operation of second-order substitution satisfies the following equation

$$
\bar{\lambda}_{X}(t)=t^{\prime}\left(\bar{\lambda}_{X}\left(t_{1}\right), \ldots, \bar{\lambda}_{X}\left(t_{n}\right)\right) .
$$

For example, suppose that $\Sigma$ consists of two binary symbols + and $*$ and a constant 1 , and $\Gamma$ consists of a binary symbol $b$, a unary one $u$ and a constant $c$. Furthermore, let $\lambda$ be given by $\ell: \Sigma \longrightarrow T_{\Gamma} \varnothing J$ as follows:

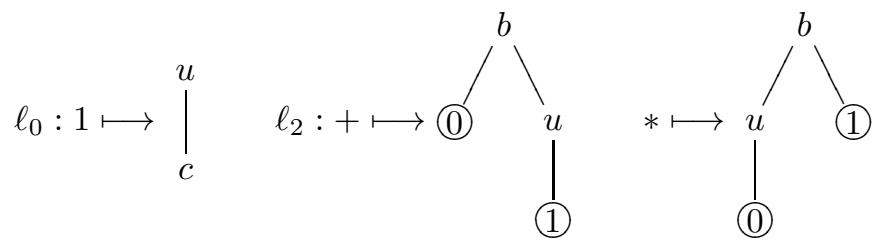

and else $\ell_{n}$ is the unique map from the empty set. For the set $X=\left\{x, x^{\prime}\right\}$, the second-order substitution morphism $\bar{\lambda}_{X}$ acts for example as follows:

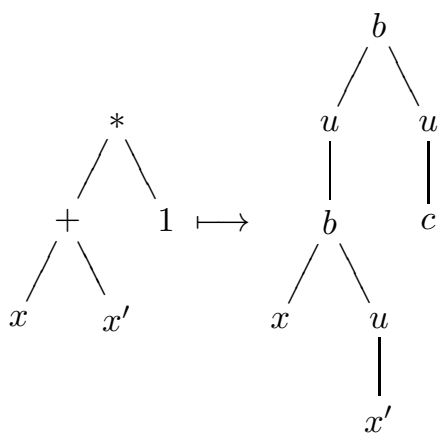




\section{5. $T^{H}$ AS Final Coalgebra Among Monads}

In this section we will state and prove some technical results which are essential for the proofs of our results on uninterpreted and interpreted program schemes. The culmination of the work comes in Corollary 5.5 .

We would like to mention that the results and proofs in Section 5.1 essentially appear in the work of Ghani et al. GLM]. However, that paper does not work in the same category as we do. Our setting is, perhaps, conceptually slightly clearer and we therefore include full proofs. We do not believe that any of our subsequent new results in Sections 6 and 7 can be obtained by simply applying the solution theorem of GLM.

We still assume that every functor $H$ we consider is an iteratable endofunctor. Recall that for each object $Y, T Y$ is a final coalgebra for the functor $H\left({ }_{-}\right)+Y$ on $\mathcal{A}$.

We are going to prove a number of results that strengthen this. First, consider the functor category $[\mathcal{A}, \mathcal{A}]$. $H$ may be regarded as a functor on this, by $F \longmapsto H \varnothing F$. We also get a related functor $H \varnothing_{-}+I d$. For the functor $T$, the value of this functor at $T$ is $H \varnothing T+I d$. So the natural transformation $\alpha^{H}: T \longrightarrow H T+I d$ of Theorem 3.15(2) may be regarded as a coalgebra structure on $T$. The proof of the following theorem is straightforward and therefore left to the reader.

Theorem 5.1. $(T, \alpha)$ is a final coalgebra for $H_{\varnothing}+I d$.

5.1. $T^{H}$ Gives a Final Coalgebra as a Monad. We next consider the subcategory of the comma-category $H /[\mathcal{A}, \mathcal{A}]$ whose objects are given by pairs $(S, \sigma: H \longrightarrow S)$, where $S$ is a monad on $\mathcal{A}$, and morphisms $h:\left(S_{1}, \sigma_{1}\right) \longrightarrow\left(S_{2}, \sigma_{2}\right)$ are monad morphisms $h: S_{1} \longrightarrow S_{2}$ such that $h \varnothing \sigma_{1}=\sigma_{2}$. We slightly abuse notation and write

$$
H / \operatorname{Mon}(\mathcal{A})
$$

for this category. For example, one object of $H / \operatorname{Mon}(\mathcal{A})$ is $(T, \kappa)$, where $\kappa=\tau \varnothing H \eta$ is the canonical natural transformation of Theorem 3.15(4). We show that $H$ determines an endofunctor $\mathcal{H}$ on this category, and that $(T, \kappa)$ is the underlying object of a final $\mathcal{H}$-coalgebra. We then extend this finality result by considering a subcategory of $H / \operatorname{Mon}(\mathcal{A})$. We keep abusing notation and denote by $H / \operatorname{CIM}(\mathcal{A})$ the category whose objects are the pairs $(S, \sigma)$, where $S$ is completely iterative (and therefore an ideal monad, see Definition 4.5(iii)) and $\sigma$ is an ideal natural transformation; the morphisms in $H / \mathbf{C I M}(\mathcal{A})$ are given by the ideal monad morphisms, see Definitions 4.3 and 4.5 . Again, $(T, \kappa)$ is an object in this category, and we show that $\mathcal{H}$ restricts to an endofunctor on $H / \mathbf{C I M}(\mathcal{A})$, and that $(T, \kappa)$ is once again a final coalgebra for $\mathcal{H}$.

Let us begin by defining $\mathcal{H}$ on objects of $H / \operatorname{Mon}(\mathcal{A})$ as the assignment

$$
\mathcal{H}:(S, \sigma) \longmapsto(H S+I d, \text { in } \varnothing H \eta),
$$

and for a morphism $h:\left(S_{1}, \sigma_{1}\right) \longrightarrow\left(S_{2}, \sigma_{2}\right)$ we let $\mathcal{H}(h)=H h+I d$.

Furthermore, notice that for any object $(S, \sigma)$ of $H / \operatorname{Mon}(\mathcal{A})$, there is a natural transformation

$$
\xi_{(S, \sigma)} \equiv H S+I d \stackrel{[\mu \varnothing \sigma S, \eta]}{\longrightarrow} S
$$

As it turns out, the $\xi_{(S, \sigma)}$ are the components of a natural transformation $\xi: \mathcal{H} \longrightarrow I d$ turning $\mathcal{H}$ into a well-copointed endofunctor on $H / \operatorname{Mon}(\mathcal{A})$.

\section{Lemma 5.2.}

(i) $\mathcal{H}$ is an endofunctor of $H / \operatorname{Mon}(\mathcal{A})$;

(ii) $\xi: \mathcal{H} \longrightarrow$ Id is a natural transformation;

(iii) The functor $\mathcal{H}$ is well-copointed. That is, $\xi: \mathcal{H} \longrightarrow$ Id is a natural transformation with $\mathcal{H} \xi_{(S, \sigma)}=$ $\xi_{\mathcal{H}(S, \sigma)}$ for all objects $(S, \sigma)$ of $H / \operatorname{Mon}(\mathcal{A})$.

Proof. (i) Given the object $(S, \sigma)$ we define a natural transformation $\nu$ as

$$
\begin{gathered}
(H S+I d)^{2}=H S(H S+I d)+H S+I d \\
\qquad H S[\mu \varnothing \sigma S, \eta]+H S+I d \\
H S S+H S+I d \\
\downarrow \downarrow \downarrow \mid[H \mu, \text { inl }]+I d \\
H S+I d
\end{gathered}
$$


It is easy to check that $(H S+I d$, inr,$\nu)$ is a monad, see $\mathrm{Mi}_{1}$, Lemma 3.4. Together with the natural transformation

$$
H \stackrel{H \eta}{\longrightarrow} H S \stackrel{\text { inl }}{\longrightarrow} H S+I d
$$

we obtain an object of $H / \operatorname{Mon}(\mathcal{A})$.

Now suppose that $h:\left(S_{1}, \sigma_{1}\right) \longrightarrow\left(S_{2}, \sigma_{2}\right)$ is a morphism in $H / \operatorname{Mon}(\mathcal{A})$. We establish below that $\mathcal{H}(h)=H h+I d$ is a monad morphism. Together with the commutativity of the diagram

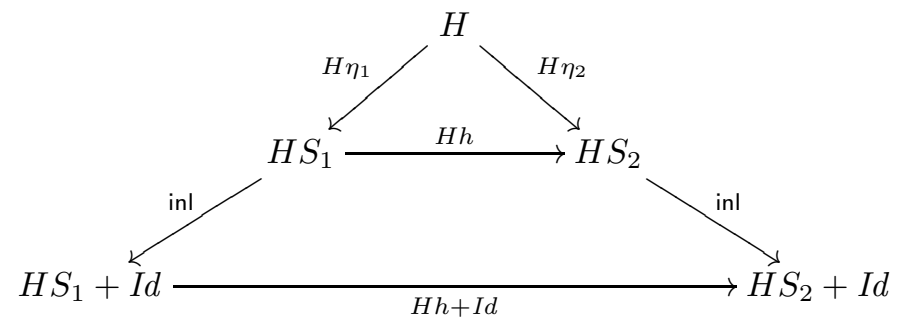

this establishes the action of $\mathcal{H}$ on morphisms. That $\mathcal{H}$ preserves identities and composition is obvious. Let us check then that $\mathcal{H}(h)$ is a monad morphism. The unit law is the commutativity of the triangle

$$
H S_{1}+\underset{\mathrm{inr}}{I d} \stackrel{{ }_{I d}^{H h+I d}}{\longrightarrow} H S_{2}+I d
$$

Thus, to complete the proof of (i) we must check that the following square commutes:

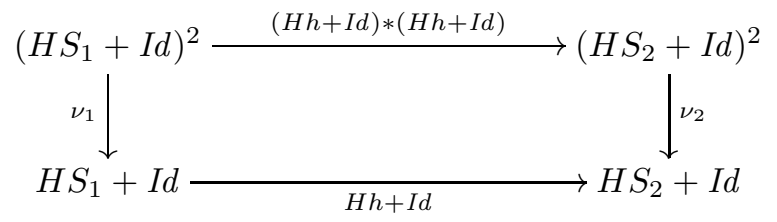

Expanding by using the definition of $\nu_{i}, i=1,2$, this is an essentially easy chase through some large diagrams using only the monad laws for the $S_{i}$, as well as the fact that $h$ is a morphism in $H / \operatorname{Mon}(\mathcal{A})$. We leave this straightforward task to the reader.

(ii) It is easy to prove that each component $\xi_{(S, \sigma)}$ is a monad morphism, see the proof of Theorem 3.1 in $\mathrm{Mi}_{1}$. Moreover, by the commutativity of the following diagram, we obtain a morphism in $H / \operatorname{Mon}(\mathcal{A})$ :

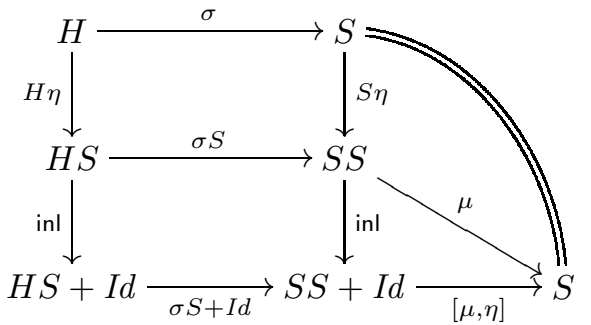

Finally, we check naturality of $\xi$. Suppose that $h:\left(S_{1}, \sigma_{1}\right) \longrightarrow\left(S_{2}, \sigma_{2}\right)$ is a morphism in $H / \operatorname{Mon}(\mathcal{A})$. Then, we must prove that the following diagram commutes:

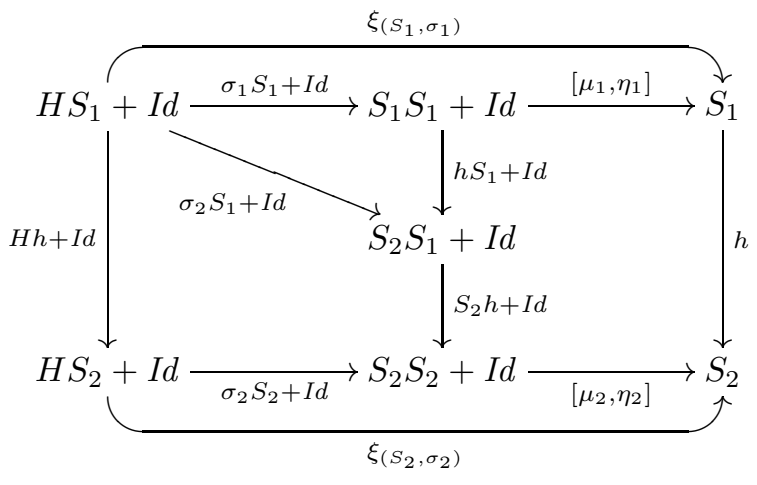


On the left we are using the naturality of $\sigma_{2}$, on the right and in the triangle we use that $h$ is a morphism of $H / \operatorname{Mon}(\mathcal{A})$.

(iii) For any object $(S, \sigma)$ we have by definition that

$$
\xi_{\mathcal{H}(S, \sigma)} \equiv[\nu \varnothing \operatorname{inl} \varnothing H \eta(H S+I d), \mathrm{inr}]: H(H S+I d)+I d \longrightarrow H S+I d .
$$

We show that this is the same as $H \xi_{(S, \sigma)}+I d$.

We consider the components of the coproduct separately. Equality on the right-hand component is obvious. For the left-hand one we shall verify the commutativity of the following diagram:

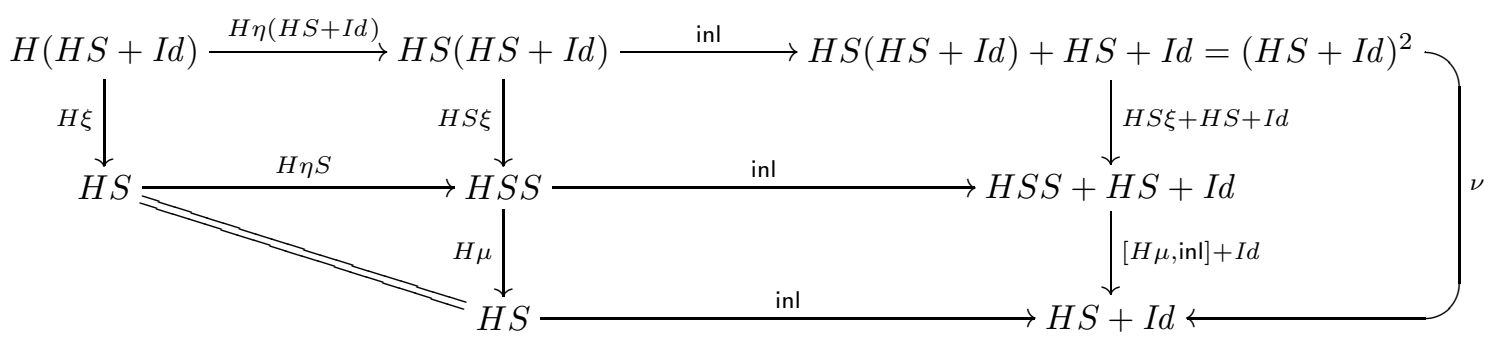

The upper and right outer edges compose to yield the left-hand component of $\xi_{\mathcal{H}(S, \sigma)}$, and the left and lower outer edges yield the left-hand component of $H \xi_{(S, \sigma)}+I d$. The desired commutativity of the outer shape follows, since all inner parts of the diagram trivially commute.

Lemma 5.3. Let $((S, \sigma), \beta)$ be an $\mathcal{H}$-coalgebra with the structure $\beta:(S, \sigma) \longrightarrow \mathcal{H}(S, \sigma)$ in $H / \operatorname{Mon}(\mathcal{A})$. Then

$$
\xi_{(S, \sigma)}: \mathcal{H}(S, \sigma) \longrightarrow(S, \sigma)
$$

is an $\mathcal{H}$-coalgebra homomorphism.

Proof. It is clear that $\mathcal{H}(S, \sigma)=(H S+I d$, inl $\varnothing H \eta)$ is an $\mathcal{H}$-coalgebra with the structure $H \beta+I d$. Also, one readily verifies that $\xi_{(S, \sigma)}$ is a morphism in $H / \operatorname{Mon}(\mathcal{A})$. From the naturality and well-copointedness of $\xi$ (see Lemma 5.2), the following square

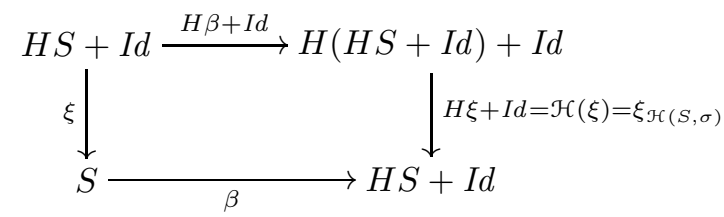

commutes.

Theorem 5.4. $((T, \kappa), \alpha)$ is a final coalgebra for the functor $\mathcal{H}$ on $H / \operatorname{Mon}(\mathcal{A})$.

Proof. Recall that the coalgebra structure $\alpha: T \longrightarrow H T+I d$ is given by the inverse of $[\tau, \eta]: H T+I d \longrightarrow T$. We prove that $\alpha$ is a morphism in $H / \operatorname{Mon}(\mathcal{A})$. Indeed, recall from Corollary 3.17 that $\tau=\mu \varnothing \kappa T$. Hence, $[\tau, \eta]=\xi_{(T, \kappa)}$. So the natural transformation $\alpha=[\tau, \eta]^{-1}$ is an inverse of a monad morphism. Thus, $\alpha$ is itself a monad morphism, and clearly we have $\alpha \varnothing \kappa=$ inl $\varnothing H \eta$.

Now suppose that $\beta:(S, \sigma) \longrightarrow \mathcal{H}(S, \sigma)$ is any $\mathcal{H}$-coalgebra. So the natural transformation $\beta: S \longrightarrow$ $H S+I d$ is a monad morphism such that $\beta \varnothing \sigma=\operatorname{inl} \varnothing H \eta^{S}$. By Theorem 5.1 $T$ is a final coalgebra on the level of endofunctors. Thus there exists a unique natural transformation $h: S \longrightarrow T$ such that the following square commutes:

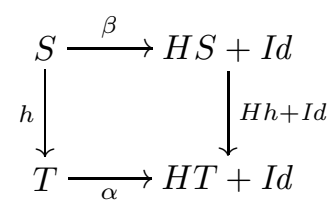

Hence our only task is to show that $h$ is a morphism in $H / \operatorname{Mon}(\mathcal{A})$. With an easy computation we establish the unit law:

$$
\begin{aligned}
h \varnothing \eta^{S} & =\alpha^{-1} \varnothing(H h+I d) \varnothing \beta \varnothing \eta^{S} & & \text { (by (5.1)) } \\
& =[\tau, \eta] \varnothing(H h+I d) \varnothing \mathrm{inr} & & \left(\text { since } \alpha^{-1}=[\tau, \eta] \text { and } \beta \varnothing \eta^{S}=\mathrm{inr}\right) \\
& =[\tau, \eta] \varnothing \mathrm{inr} & & \\
& =\eta & & \text { (computation with inr) }
\end{aligned}
$$


and from this it follows that

$$
\begin{aligned}
& h \varnothing \sigma=\alpha^{-1} \varnothing(H h+I d) \varnothing \beta \varnothing \sigma \quad \text { (by (5.1)) } \\
& =[\tau, \eta] \varnothing(H h+I d) \varnothing \operatorname{inl} \varnothing H \eta^{S} \quad \text { (since } \alpha^{-1}=[\tau, \eta] \\
& \text { and } \left.\beta \varnothing \sigma=\operatorname{inl} \varnothing H \eta^{S}\right) \\
& =\tau \varnothing H h \varnothing H \eta^{S} \quad \text { (composition with inl) } \\
& =\tau \varnothing H \eta \quad\left(\text { since } h \varnothing \eta^{S}=\eta\right) \\
& =\kappa \quad \text { (by Theorem } 3.15(4) \text { ). }
\end{aligned}
$$

Finally, we check that the following square commutes:

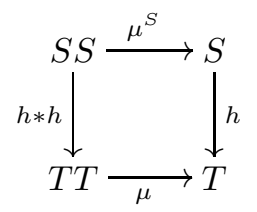

In order to do this we establish below that the arrows in this square are coalgebra homomorphisms. Then (5.2) is immediate because $T$ is a final coalgebra for the functor $H \varnothing_{-}+I d:[\mathcal{A}, \mathcal{A}] \longrightarrow[\mathcal{A}, \mathcal{A}]$. Firstly, we need to specify the coalgebra structures on the objects in (5.2). For $S$ and $T$, we of course use $\beta$ and $\alpha$, respectively. For $S S$, we use the following coalgebra structure

$$
S S \stackrel{\beta * \beta}{\longrightarrow}(H S+I d)^{2}=H S(H S+I d)+H S+I d \stackrel{\left[H S \xi, H \eta^{S} S\right]+I d}{\longrightarrow} H S S+I d
$$

We shall now establish that $\mu^{S}: S S \longrightarrow S$ is a coalgebra homomorphism. That is, we prove that the following diagram commutes:

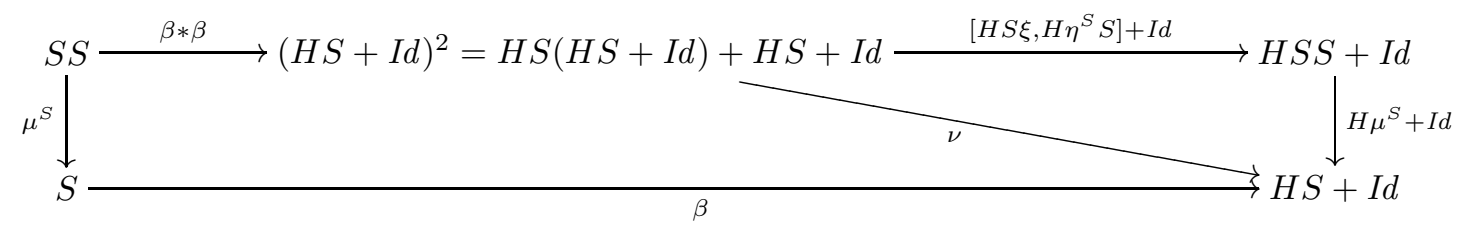

The left-hand part commutes since $\beta$ is a monad morphism and the right-hand one is the definition of $\nu$ (use that $H \mu^{S} \varnothing H \eta S=1_{H S}$ ). Similarly, there is a coalgebra structure on $T T$ such that $\mu: T T \longrightarrow T$ is a coalgebra homomorphism. Finally, we show that $h * h: S S \longrightarrow T T$ is a coalgebra homomorphism. To this end we shall verify the commutativity of the diagram below:

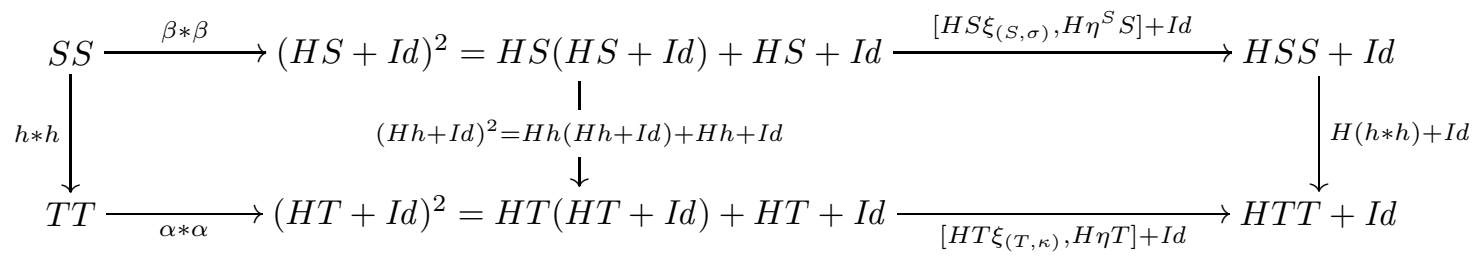

We write $(H h+I d)^{2}$ to abbreviate $(H h+I d) *(H h+I d)$. So by the double interchange law (2.3) and by (5.1) the left-hand square commutes. We consider the right-hand square componentwise: The right-hand component is obvious. For the middle component we remove $H$ and obtain the following commutative square:

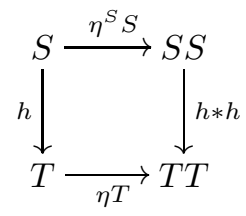


Finally, for the left-hand component we remove $H$ again to obtain

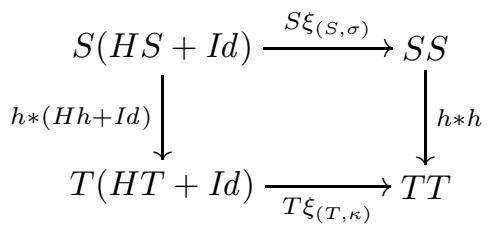

By the double interchange law (2.3) and the fact that $F \xi=i d_{F} * \xi$, it suffices to check that the square

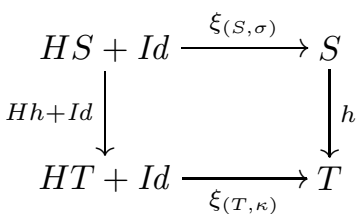

commutes. Notice that we are not entitled to use naturality of $\xi$ here, since we do not yet know that $h$ is a monad morphism. Instead, we invoke the finality of $T$ and show that all arrows in the above square are coalgebra homomorphisms for the functor $H \varnothing_{-}+I d$ on $[\mathcal{A}, \mathcal{A}]$. Indeed, since $h$ is a coalgebra homomorphism, so is $H h+I d$; the other two morphisms are coalgebra homomorphisms by Lemma 5.3 This completes the proof.

5.2. $T^{H}$ Gives a Final Coalgebra as a Completely Iterative Monad. The next result is the main technical tool for our treatment of recursive program schemes in Section 6 below. Recall that we denote by $H / \operatorname{CIM}(\mathcal{A})$ the category whose objects are the pairs $(S, \sigma)$, where $S$ is a completely iterative monad and $\sigma$ is an ideal natural transformation; the morphisms in $H / \mathbf{C I M}(\mathcal{A})$ are given by the ideal monad morphisms. So $H / \operatorname{CIM}(\mathcal{A})$ is a subcategory of $H / \operatorname{Mon}(\mathcal{A})$. We show in Corollary 5.5 just below that $((T, \kappa), \alpha)$ is a final coalgebra for the same functor $\mathcal{H}$ that we have been working with. In the language of Theorem 5.4 , the main point of the corollary is that if $\beta: S \longrightarrow H S+I d$ is an ideal monad morphism which is a coalgebra for $\mathcal{H}$ on completely iterative monad $S$, then the morphism into $T$ may be taken to be an ideal monad morphism as well.

Corollary 5.5. $\mathcal{H}$ restricts to an endofunctor on $H / \mathbf{C I M}(\mathcal{A})$, and $((T, \kappa), \alpha)$ is a final $\mathcal{H}$-coalgebra in $H / \operatorname{CIM}(\mathcal{A})$.

Proof. Lemma 3.5 of $\mathrm{Mi}_{1}$ gives a result propagating the complete iterativeness of monads: for any completely iterative monad $S$ with ideal $\iota: S^{\prime} \longleftrightarrow S$, and any natural transformation $\sigma: H \longrightarrow S$ such that $\sigma=\iota \varnothing \sigma^{\prime}$ for some $\sigma^{\prime}: H \longrightarrow S^{\prime}$, the monad $H S+I d$ is completely iterative, too-its ideal is inl : $H S \hookrightarrow H S+I d$, of course. Moreover, for any monad morphism $h, \mathcal{H}(h)=H h+I d$ is an ideal monad morphism. Hence $\mathcal{H}$ restricts to an endofunctor on $H / \operatorname{CIM}(\mathcal{A})$, which by abuse of notation we denote by $\mathcal{H}$ again.

By Theorem 4.6, $(T, \kappa)$ lies in $H / \operatorname{CIM}(\mathcal{A})$, and it is clear that the coalgebra structure $\alpha=[\tau, \eta]^{-1}$ is an ideal monad morphism: the restriction of $\alpha$ to the ideals is $i d: H T \longrightarrow H T$. Now suppose that $\beta:(S, \sigma) \longrightarrow\left(H S+I d\right.$, inl $\left.\varnothing H \eta^{S}\right)$ is an $\mathcal{H}$-coalgebra; that is, $\beta$ is an ideal monad morphism between completely iterative monads such that the following square

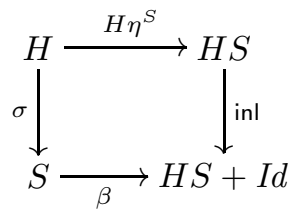

commutes. By Theorem [5.4 we obtain a unique coalgebra homomorphism $h:(S, \sigma) \longrightarrow(T, \kappa)$ in $H / \operatorname{Mon}(\mathcal{A})$. Our only task is to check that $h$ is an ideal monad morphism, so that it is a morphism in $H / \operatorname{CIM}(\mathcal{A})$. Since $\beta$ is an ideal monad morphism, there is some $\beta^{\prime}: S^{\prime} \longrightarrow H S$ such that the upper-left 
square below commutes:

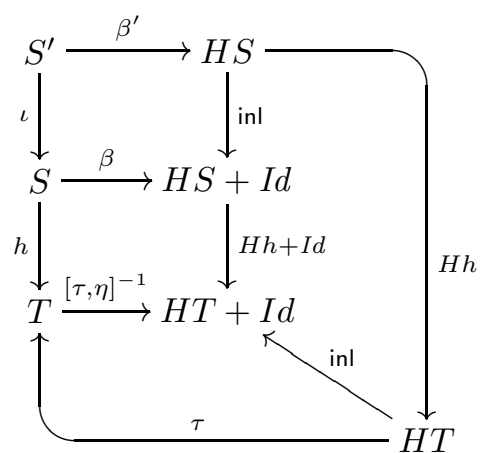

We verify that the rest of the interior regions commute. The middle square commutes since $h$ is a coalgebra homomorphism. The two other parts are obvious. Hence the outer shape commutes, proving that $H h \emptyset \beta^{\prime}$ : $S^{\prime} \longrightarrow H T$ is a restriction of $h$ to the ideal $S^{\prime}$ of $S$.

\section{Uninterpreted Recursive Program Schemes}

In the classical treatment of recursive program schemes, one gives an uninterpreted semantics to systems like (1.1) which are in Greibach normal form, i. e., every tree on the right-hand side of the system has as its head symbol a symbol from the signature $\Sigma$ of givens. The semantics assigns to each of the recursively defined operation symbols a $\Sigma$-tree. These trees are obtained as the result of unfolding the recursive specification of the RPS. We illustrated this with an example in (1.7) in the Introduction.

We have seen in Section 2 that $\Sigma$-trees are the carrier of a final coalgebra for the signature functor $H_{\Sigma}$. It is the universal property of this final coalgebra which allows one to give a semantics to the given RPS. Using the technical tools developed in Section 5 we will now provide a conceptually easy and general way to give an uninterpreted semantics to recursive program schemes considered more abstractly as natural transformations, see our discussion in Section 2.3

But before we do this, we need to say what a solution of an RPS is. To do this we use the universal property of the monads $T^{H}$ as presented in Section 4, The universal property provides an abstract version of second-order substitution.

Here are our central definitions, generalizing recursive program schemes from signatures to completely iterative monads.

Definition 6.1. Let $V$ and $H$ be endofunctors on $\mathcal{A}$. A recursive program scheme (or $R P S$, for short) is a natural transformation

$$
e: V \longrightarrow T^{H+V}
$$

We sometimes call $V$ the variables, and $H$ the givens.

The RPS $e$ is called guarded if there exists a natural transformation

$$
f: V \longrightarrow H T^{H+V}
$$

such that the following diagram commutes:

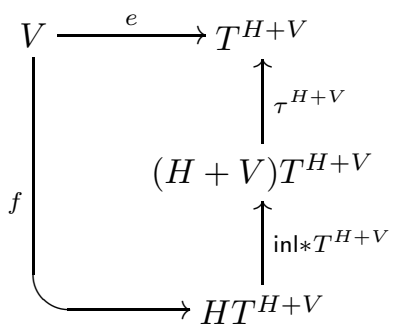

A solution of $e$ is an ideal natural transformation $e^{\dagger}: V \longrightarrow T^{H}$ such that the following triangle commutes:

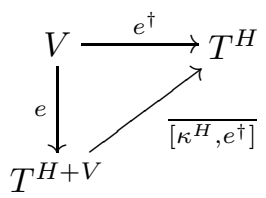


Remark 6.2. Recall that $\overline{\left[\kappa^{H}, e^{\dagger}\right]}$ is the unique ideal monad morphism extending $\sigma=\left[\kappa^{H}, e^{\dagger}\right]: H+$ $V \longrightarrow T^{H}$ (see Theorem 4.6). Observe that therefore it is important to require that $e^{\dagger}$ be an ideal natural transformation, since otherwise $\bar{\sigma}$ is not defined.

Remark 6.3. $\quad$ (i) From Section 2.3, our definition is a generalization of the classical notion of RPS (to the category theoretic setting), and it extends the classical work as well by allowing infinite trees on the right-hand sides of equations.

(ii) The classical notion of Greibach normal form (see [C]) requires that a system of formal equations such as (2.5) has the roots of all right-hand sides labelled in $\Sigma$. Equivalently, the corresponding natural transformation $\Phi \longrightarrow T_{\Sigma+\Phi} \varnothing J$ factors as $\Phi \longrightarrow H_{\Sigma} T_{\Sigma+\Phi} \varnothing J \longrightarrow T_{\Sigma+\Phi} \varnothing J$. Translated along the bijective correspondence (2.4) that means that a systems (2.5) is in Greibach normal form iff the corresponding RPS $e: H_{\Phi} \longrightarrow T^{H_{\Sigma}+H_{\Phi}}$ is guarded in the sense of Definition 6.1.

(iii) Suppose that $H=H_{\Sigma}$ and $V=H_{\Phi}$ are signature functors of Set, and consider the recursive program scheme $e: H_{\Phi} \longrightarrow T^{H_{\Sigma}+H_{\Phi}}$ as a set of formal equations as in (2.5). Then for any set $X$ of syntactic variables the $X$-component $e_{X}^{\dagger}: H_{\Phi} X \longrightarrow T_{\Sigma} X$ of a solution assigns to any flat tree $\left(f, x_{1}, \ldots, x_{n}\right)=(f, \vec{x})$ from $H_{\Phi} X$ a $\Sigma$-tree over $X$. The commutativity of the triangle (6.2) gives the following property of solutions: apply to the right-hand side $t^{f}(\vec{x})$ of $f(\vec{x})$ in the given RPS the second-order substitution that replaces each operation symbol of $\Phi$ by its solution, and each

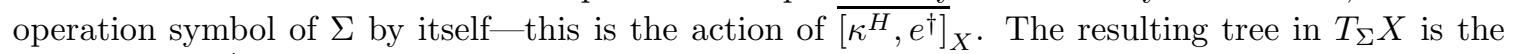
same tree as $e_{X}^{\dagger}(f, \vec{x})$.

(iv) Any guarded equation morphism $e: X \longrightarrow T(X+Y)$ w.r.t. the free completely iterative monad $T=T^{H}$ (see Definition 4.5) can be turned into a guarded recursive program scheme with the variables $C_{X}$ and the givens $H+C_{Y}$, where $C_{X}$ and $C_{Y}$ denote constant functors. From our result in Theorem 6.5 below, one obtains a unique solution of that recursive program scheme, and one can prove that this unique solutions yields the unique solution of $e$ in the sense of Definition 4.5. The non-trivial proof of this fact is left to the future paper $\mathrm{MM}_{2}$.

Example 6.4. Let us now present two classical RPSs as well as an example of RPS which is not captured with the classical setting.

(i) Recall from the Introduction the formal equations of (1.1) and the ubiquitous (1.3) defining the factorial function. As explained in Example 2.13, these give rise to recursive program schemes. Since both (1.1) and (1.3) are in Greibach normal form, we obtain two guarded RPSs in the sense of Definition 6.1 above. For example, the RPS obtained from (1.3) comes from the natural transformation $\Phi \longrightarrow H_{\Sigma} T_{\Sigma+\Phi} \varnothing J \longleftrightarrow T_{\Sigma+\Phi} \varnothing J$ with

$$
f \longmapsto \text { (ifzero, }(0, \text { one, } f(\operatorname{pred}(0)) * 0)) \in \Sigma_{3} \times\left(T_{\Sigma+\Phi}\{0\}\right)^{3} \longleftrightarrow H_{\Sigma} T_{\Sigma+\Phi}\{0\},
$$

and similarly for (1.1).

(ii) Sometimes one might wish to recursively define new operations from old ones where the new operations should satisfy certain extra properties automatically. We demonstrate this with an RPS recursively defining a new operation which is commutative. Suppose the signature $\Sigma$ of givens consists of a ternary symbol $F$ and a unary one $G$. Let us assume that we want to require that $F$ satisfies the equation $F(x, y, z)=F(y, x, z)$ in any interpretation. This is modeled by the endofunctor $H X=X^{3} / \sim+X$, where $\sim$ is the smallest equivalence on $X^{3}$ with $(x, y, z) \sim(y, x, z)$. To be an $H$-algebra is equivalent to being an algebra $A$ with a unary operation $G_{A}$ and a ternary one $F_{A}$ satisfying $F_{A}(x, y, z)=F_{A}(y, x, z)$. Suppose that one wants to define a commutative binary operation $\varphi$ by the formal equation

$$
\varphi(x, y) \approx F(x, y, \varphi(G x, G y)) .
$$

To do it we express $\varphi$ by the endofunctor $V$ assigning to a set $X$ the set $\{\{x, y\} \mid x, y \in X\}$ of unordered pairs of $X$. It is not difficult to see that the formal equation (6.3) gives rise to a guarded RPS $e: V \longrightarrow T^{H+V}$. In fact, to see the naturality use the description of the terminal coalgebra $T^{H+V} Y$ given in [AM], see Example 2.7(i). Notice that in the classical setting we are unable to demand that (the solution of) $\varphi$ be a commutative operation at this stage: one would use general facts to obtain a unique solution, and then one would need to devise a special argument to verify commutativity of that solution. Once again, our general theory ensures that any solution of our RPS will be commutative.

The main result of this section is the following theorem. Before we present its proof below let us illustrate the result with a few examples. 
Theorem 6.5. Every guarded recursive program scheme has a unique solution.

Examples 6.6. We present here the solutions of the RPSs of Example 6.4.

(i) The unique solution of the RPS induced by the equations (1.1) is an ideal natural transformation $e^{\dagger}: H_{\Phi} \longrightarrow T^{H_{\Sigma}}$. Equivalently, we have a natural transformation $\Phi \longrightarrow T_{\Sigma} \varnothing J$, see (2.4). That is, the solution $e^{\dagger}$ is essentially given by two $\Sigma$-trees (one for each of the operation symbols $\varphi$ and $\psi$ ) over a singleton set, say $\{x\}$. It follows from the proof of Theorem 6.5 that those $\Sigma$-trees are the ones given in (1.2), see Example 6.14 below.

Similarly, the unique solution of the RPS induced by the equation (1.3) is essentially given by the $\Sigma$-tree over the set $\{n\}$ below:

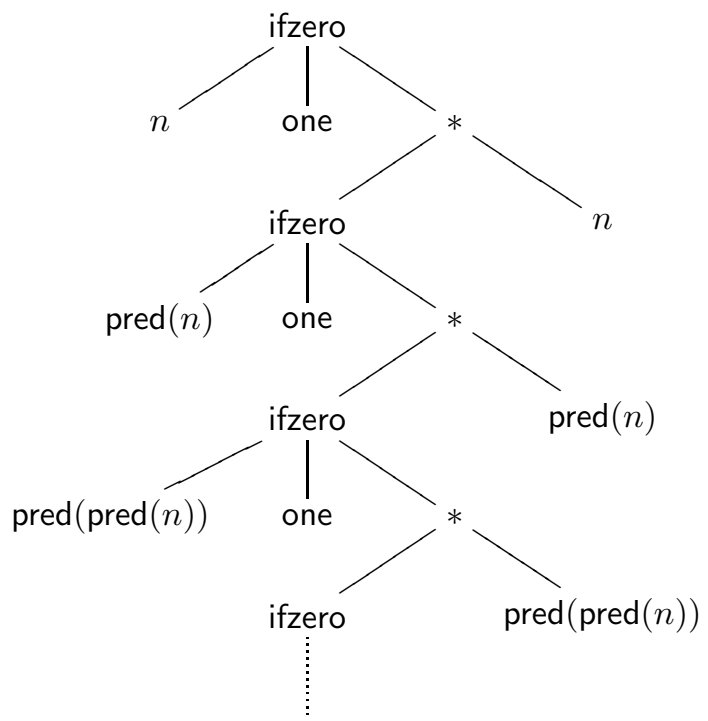

Notice that the nodes labelled by a term correspond to appropriately labelled finite subtrees.

(ii) We continue example 6.4(ii) and describe the uninterpreted solution of the guarded RPS $e$ arising from the formal equation (6.3) defining a commutative operation. The components of $e_{X}^{\dagger}: V X \longrightarrow$ $T^{H} X$ assign to an unordered pair $\{x, y\}$ in $V X$ the tree

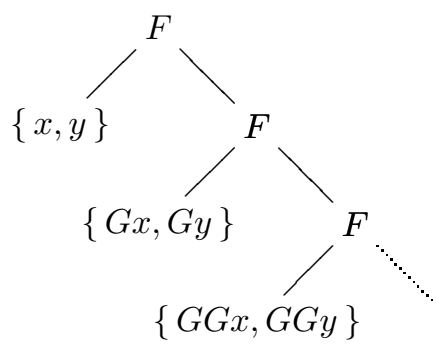

where for every node labelled by $F$ the order of the first two children cannot be distinguished; we indicate this with set-brackets in the picture above.

Remark 6.7. Notice that in the classical setting not every recursive program scheme which has a unique solution needs to be in Greibach normal form. For example, consider the system formed by the first equation in (1.1) and by the equation $\psi(x) \approx \varphi(\psi(x))$. This system gives rise to an unguarded RPS. Thus, Theorem6.5 does not provide a solution of this RPS. However, the system is easily rewritten to an equivalent one in Greibach normal form which gives a guarded RPS that we can uniquely solve using our Theorem 6.5]

The rest of this section is devoted to the proof of Theorem 6.5. We illustrate each crucial step with the help of our examples. Before we turn to the proof of the main theorem we need to establish some preliminary lemmas.

Lemma 6.8. Let $H$ and $K$ be endofunctors on $\mathcal{A}$. Suppose we have objects $(S, \sigma)$ of $H / \mathbf{M o n}(\mathcal{A})$ and $(R, \rho)$ of $K / \operatorname{Mon}(\mathcal{A})$. Let $n: H \longrightarrow K$ be a natural transformation and let $m: S \longrightarrow R$ be a monad morphism 
such that the following square

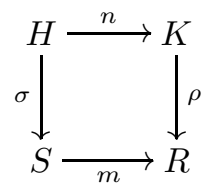

commutes. Then $n * m+I d: H S+I d \longrightarrow K R+I d$ is a monad morphism such that the following square

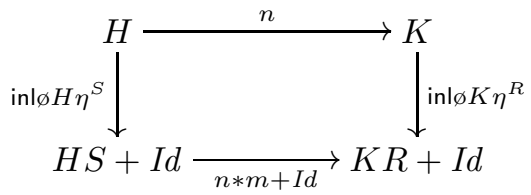

commutes.

Proof. Naturality and the unit law are clear, and the preservation of the monad multiplication is a straightforward diagram chasing argument which we leave to the reader.

Lemma 6.9. Consider any guarded RPS e, with a factor $f$ as in (6.1). There exists a unique (ideal) monad morphism

$$
\widehat{e}: T^{H+V} \longrightarrow T^{H+V}
$$

such that the following triangle commutes:

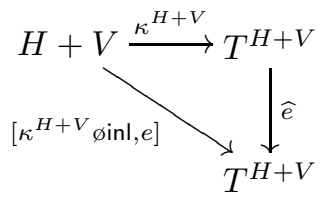

There is also a unique (ideal) monad morphism

$$
\bar{e}: T^{H+V} \longrightarrow H T^{H+V}+I d
$$

such that the following diagram commutes:

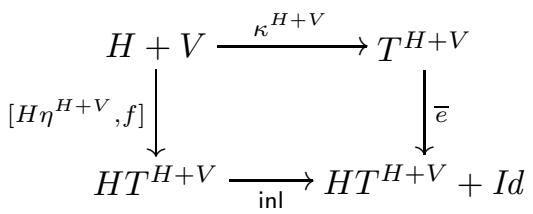

Proof. We get $\widehat{e}$ from Theorem 4.6. Indeed, it is easily checked that

$$
\left[\kappa^{H+V} \varnothing \operatorname{inl}, e\right]: H+V \longrightarrow T^{H+V}
$$

is an ideal natural transformation using that $e$ is guarded. As for $\bar{e}$, recall that $H$ "embeds" into $T^{H+V}$ via the natural transformation

$$
H \stackrel{\text { inl }}{\longrightarrow} H+V \stackrel{\kappa^{H+V}}{\longrightarrow} T^{H+V} .
$$

Since $\kappa^{H+V}$ is an ideal natural transformation, so is this one. Hence we have an object $\left(T^{H+V}, \kappa^{H+V} \varnothing \operatorname{inl}\right)$ of $H / \mathbf{C I M}(\mathcal{A})$. It follows from Corollary 5.5 that $H T^{H+V}+I d$ carries the structure of a completely iterative monad. The natural transformation inlø[H$\left.\eta^{H+V}, f\right]: H+V \longrightarrow H T^{H+V}+I d$, see (6.5), is obviously ideal. Thus, we obtain $\bar{e}$ as desired from another application of Theorem 4.6

Remark 6.10. In the leading example of a classical RPS for given signatures, the formation of the morphism $\bar{e}$ corresponds to the formation of a flat system of equations, where for every tree there is a formal variable. More precisely, suppose we have signatures $\Sigma$ and $\Phi$, and an RPS as in (2.5) which is in Greibach normal form. The component of $\bar{e}$ at some set $X$ of syntactic variables can be seen as a set of formal equations. Here is a description of $\bar{e}_{X}$ : For every tree $t \in T_{\Sigma+\Phi} X$, we have a formal variable $\underline{t}$. And for each formal variable we have one formal equation:

$$
\begin{array}{ll}
\underline{t} \approx x & \text { if } t \text { is a single node tree with root label } x \in X, \text { or } \\
\underline{t} \approx \sigma\left(\underline{t_{1}}, \ldots, \underline{t_{n}}\right) & \text { for some } n \in \mathbb{N} \text { and some } \sigma \in \Sigma_{n} \text { otherwise, }
\end{array}
$$


where the tree $s=\sigma\left(t_{1}, \ldots, t_{n}\right)$ is the result of the following second-order substitution applied to $t$ : every symbol of $\Phi$ is substituted by its right-hand side in the given RPS, and every symbol of $\Sigma$ by itself. Since the given RPS is guarded, the head symbol of $s$ is a symbol of $\Sigma$ for all trees $t$.

Example 6.11. For the guarded RPS of (1.1) the flat system obtained from $\bar{e}$ for $X=\{x\}$ includes the equations of the system (1.7) from the Introduction.

We also give a fragment of the flat system obtained as the extension of the RPS (1.3), see also Example 6.4(i). Here the set of syntactic variables is $X=\{n\}$, and the formal equations described by $\bar{e}_{X}$ include the following ones:

$$
\begin{aligned}
& \underline{f(n)} \approx \text { ifzero }(\underline{n}, \text { one }, \underline{f(\operatorname{pred}(n)) * n)} \\
& \underline{n} \approx n \\
& \text { one } \approx \text { one } \\
& \underline{f(\operatorname{pred}(n))} \approx \text { ifzero }(\operatorname{pred}(n), \text { one, } f(\operatorname{pred}(\operatorname{pred}(n))) * \operatorname{pred}(n)) \\
& \underline{f(\operatorname{pred}(n)) * n} \approx \underline{\text { ifzero }(\operatorname{pred}(n), \text { one, } f(\operatorname{pred}(\operatorname{pred}(n))) * \operatorname{pred}(n))} * \underline{n} \\
& \text { : }
\end{aligned}
$$

Lemma 6.12. The following diagram commutes:

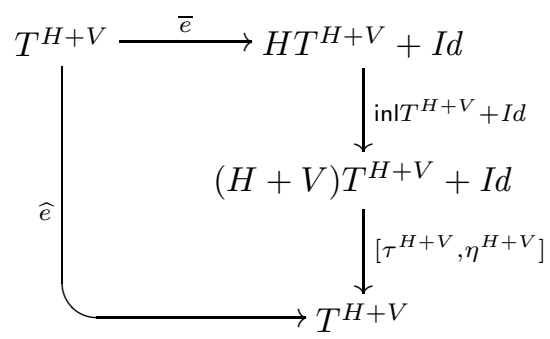

Proof. By the first part of Lemma 6.9] it suffices to show that the composite in the above diagram is an ideal monad morphism extending $\left[\kappa^{H+V} \varnothing \mathrm{inl}, e\right]$. For the extension property, we shall prove that the diagram below commutes, where we write $T$ for $T^{H+V}$ :

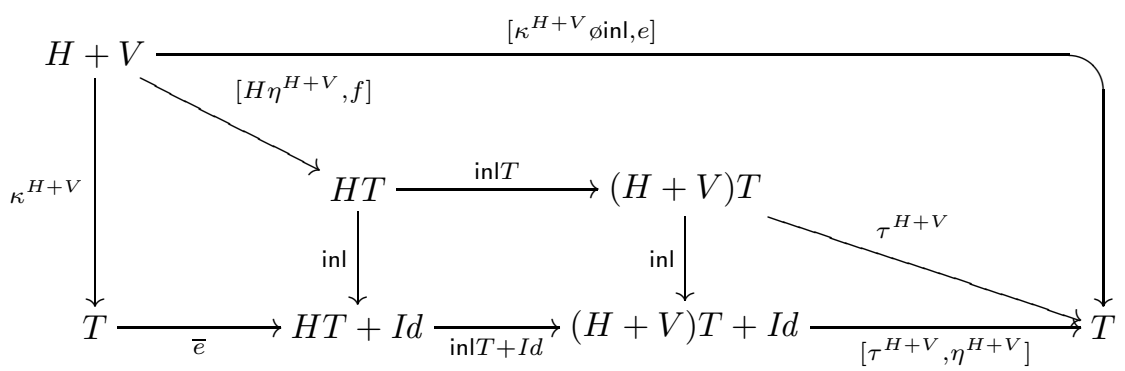

Indeed, the left-hand part commutes by Lemma 6.9 For the left-hand component of the upper part notice that, using the definitions of inl $* \eta^{H+V}$ and $\kappa^{H+V}$,

$$
\tau^{H+V} \varnothing \operatorname{inl} T \varnothing H \eta^{H+V}=\tau^{H+V} \varnothing(H+V) \eta^{H+V} \varnothing \operatorname{inl}=\kappa^{H+V} \varnothing \text { inl . }
$$

The right-hand component of this part commutes since $e$ is guarded, see (6.1), and the remaining parts are trivial.

We show that all parts of the lower edge in the above diagram are monad morphisms. For $\bar{e}$, see Lemma 6.9 , For inl $* T+I d$, apply Lemma 6.8 to $n=$ inl and $m=1_{T}$. And the for the last part, $[\tau, \eta]=[\mu \varnothing \kappa T, \eta]$, notice that it is the component at $\left(T^{H+V}, \kappa^{H+V}\right)$ of the natural transformation $\xi$ of Lemma 5.2 applied to $(H+V) / \operatorname{Mon}(\mathcal{A})$.

Proof of Theorem 6.5 (see page [32). Consider $\bar{e}$ from Lemma 6.9 It is a coalgebra structure for the functor

$$
\mathcal{H}: H / \mathbf{C I M}(\mathcal{A}) \longrightarrow H / \mathbf{C I M}(\mathcal{A}) \text {. }
$$

In fact, $\bar{e}$ is a morphism in $H / \mathbf{C I M}(\mathcal{A})$; it is an ideal monad morphism and by (6.5) we have

$$
\bar{e} \varnothing \kappa^{H+V} \varnothing \mathrm{inl}=\operatorname{inl} \varnothing\left[H \eta^{H+V}, f\right] \varnothing \operatorname{inl}=\operatorname{inl} \varnothing H \eta^{H+V} .
$$


Now apply Corollary 5.5 to obtain a unique $\mathcal{H}$-coalgebra homomorphism from the above coalgebra $\bar{e}$ to the final one. In more detail, we obtain a unique ideal monad morphism $h: T^{H+V} \longrightarrow T^{H}$ such that the following diagram commutes:

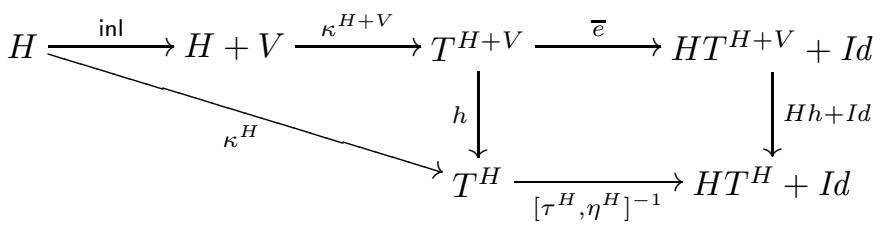

Now let

$$
e^{\dagger} \equiv V \stackrel{\mathrm{inr}}{\longrightarrow} H+V \stackrel{\kappa^{H+V}}{\longrightarrow} T^{H+V} \stackrel{h}{\longrightarrow} T^{H} .
$$

We shall prove that $e^{\dagger}$ uniquely solves $e$.

(a) $e^{\dagger}$ is a solution of $e$. Since $h$ is an ideal monad morphism and $\kappa^{H+V}$ is an ideal natural transformation, we see that $e^{\dagger}$ is an ideal natural transformation. Next observe that by definition we have

$$
h=\overline{\left[\kappa^{H}, e^{\dagger}\right]},
$$

and we also get

$$
\begin{aligned}
& e^{\dagger}=h \varnothing \kappa^{H+V} \varnothing \mathrm{inr} \quad \text { (by (6.9)) } \\
& =\left[\tau^{H}, \eta^{H}\right] \varnothing(H h+I d) \varnothing \bar{e} \varnothing \kappa^{H+V} \varnothing \mathrm{inr} \quad(\text { by }(\underline{6.8})) \\
& =\left[\tau^{H}, \eta^{H}\right] \varnothing(H h+I d) \varnothing \operatorname{inl} \varnothing f \quad \text { (by }(\underline{6.5}) \\
& =\tau^{H} \varnothing H h \varnothing f .
\end{aligned}
$$

Now we are ready to verify that the following diagram commutes:

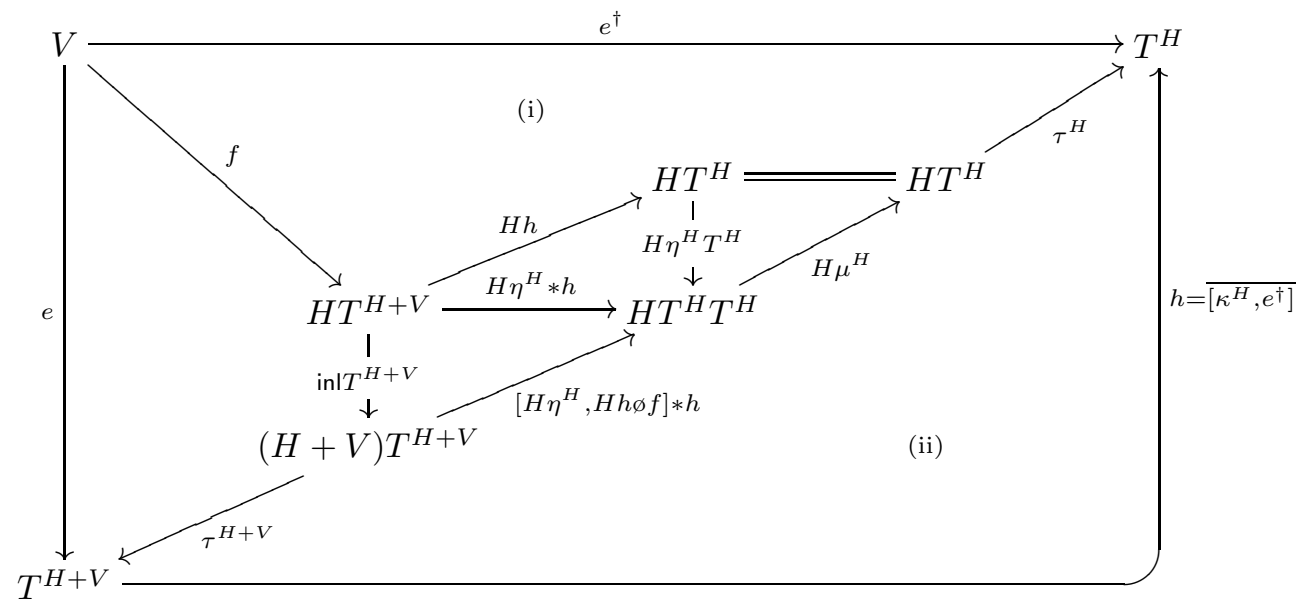

Indeed, part (i) commutes by (6.10). For part (ii), observe first that from Theorem 3.15 (4) and (6.10) we get the equation

$$
\left[\kappa^{H}, e^{\dagger}\right]=H+V \stackrel{\left[H \eta^{H}, H h \varnothing f\right]}{\longrightarrow} H T^{H} \stackrel{\tau^{H}}{\longrightarrow} T^{H} .
$$

Now apply Lemma 4.7 to $H+V$ and $H$ and $\sigma=\left[\kappa^{H}, e^{\dagger}\right.$. The other parts of (6.11) are obvious.

(b) Uniqueness of solutions. Suppose that $s: V \longrightarrow T^{H}$ is a solution of $e$. Since solutions are ideal natural transformations by definition, there exists a natural transformation $s^{\prime}: V \longrightarrow H T^{H}$ such that $s=\tau^{H} \varnothing s^{\prime}$. We shall show below that the ideal monad morphism $h$ from (6.8) is equal to

$$
\overline{\left[\kappa^{H}, s\right]}: T^{H+V} \longrightarrow T^{H}
$$

using coinduction. So we show that $\overline{\left[\kappa^{H}, s\right]}$ is a coalgebra homomorphism. Then, since $\left(T^{H}, \kappa^{H}\right)$ is a final $\mathcal{H}$-coalgebra, we can conclude that $h=\overline{\left[\kappa^{H}, s\right]}$ and therefore

$$
e^{\dagger}=\overline{\left[\kappa^{H}, e^{\dagger}\right]} \varnothing e=h \varnothing e=\overline{\left[\kappa^{H}, s\right]} \varnothing e=s,
$$

where the last equality holds since $s$ is a solution of $e$. 
For the coinduction argument, we replace $h$ in Diagram $\left[6.8\right.$ by $\overline{\left[\kappa^{H}, s\right]}$ and check that the modified diagram commutes. In fact, for the left-hand triangle we obtain:

$$
\overline{\left[\kappa^{H}, s\right]} \varnothing \kappa^{H+V} \varnothing \mathrm{inl}=\left[\kappa^{H}, s\right] \varnothing \operatorname{inl}=\kappa^{H} .
$$

To verify the modified version of the right-hand square of (6.8), we use the freeness of the completely iterative monad $T^{H+V}$. Thus, it is sufficient that this diagram of ideal monad morphisms commutes when precomposed with the universal arrow $\kappa^{H+V}$. Furthermore we consider the components of the coproduct $H+V$ separately. Let us write $x$ as a short notation for $\overline{\left[\kappa^{H}, s\right]}$. Then, for the left-hand coproduct component we obtain the following equations:

$$
\begin{array}{rlrl}
{\left[\tau^{H}, \eta^{H}\right] \varnothing(H x+I d) \varnothing \bar{e} \varnothing \kappa^{H+V} \varnothing \operatorname{inl}} & & \\
& =\left[\tau^{H}, \eta^{H}\right] \varnothing(H x+I d) \varnothing \operatorname{inl} \varnothing H \eta^{H+V} & & \text { (see (6.7)) } \\
& =\tau^{H} \varnothing H x \varnothing H \eta^{H+V} & & \\
& =\tau^{H} \varnothing H \eta^{H} & & \left(\text { since } x \varnothing \eta^{H+V}=\eta^{H}\right) \\
& =\kappa^{H} & & \text { (see Theorem 3.15(4)) } \\
& =x \varnothing \kappa^{H+V} \varnothing \mathrm{inl} & & \text { (see (6.14) })
\end{array}
$$

In order to prove that the right-hand component commutes, we use a diagram similar to Diagram (6.11). Just replace in (6.11) $H h \varnothing f$ by $s^{\prime}$, all other occurrences of $h$ by $x$, and $e^{\dagger}$ by s. We prove that part (i) in this modified diagram commutes. In fact, this follows from the fact that all other parts and the outside square commute: the outside square commutes since $s$ is a solution of $e$; part (ii) in the modified diagram commutes by Lemma 4.7 again; and all other parts are clear. Thus, we proved that the following equation holds:

$$
s=\tau^{H} \varnothing H x \varnothing f .
$$

From this we obtain the equations

$$
\begin{aligned}
& {\left[\tau^{H}, \eta^{H}\right] \varnothing(H x+I d) \varnothing \bar{e} \varnothing \kappa^{H+V} \varnothing \mathrm{inr}} \\
& \left.=\tau^{H} \varnothing H x \varnothing f \quad \text { (similar to last three lines of (6.10) }\right) \\
& =s \quad \text { (by 6.15) } \\
& =x ø \kappa^{H+V} \varnothing \operatorname{inr} \quad\left(\text { since } x=\overline{\left[\kappa^{H}, s\right]}\right) .
\end{aligned}
$$

This establishes that $h$ from $(6.9)$ is equal to $\overline{\left[\kappa^{H}, s\right]}$ from (6.13).

Remark 6.13. Recall that the formation of $\bar{e}$ corresponds, in the leading example of an RPS for given signatures, to the formation of a flat system of equations, see Remark 6.10. Now the map $h_{X}$ of (6.8) assigns to every variable $\underline{t} \in T_{\Sigma+\Phi} X$ of the flat system the $\Sigma$-tree given by unfolding the recursive specification given by this flat system, i. e., $h_{X}$ is the unique solution of the flat equation morphism $\bar{e}_{X}$ in the cia $T_{\Sigma} X$.

Example 6.14. In equations (1.1) in the beginning of this paper, we introduced a guarded RPS as it would be classically presented. It induces an RPS (in our sense), as discussed in Example 6.4(i). The unique solution of the flat equation morphism $\bar{e}_{X}$ corresponding to the flat system described in Example 6.11 is $h_{X}: T_{\Sigma+\Phi} X \longrightarrow T_{\Sigma} X$ (see also (1.7) from the Introduction). The definition of $e^{\dagger}$ in (6.9) means that we only consider the solution for the formal variables $\varphi(x)$ and $\psi(x)$ in that system. These solutions are precisely the $\Sigma$-trees (1.2) as described in Example 6.6(i).

Similarly, for the guarded RPS induced by (1.3), the solution is obtained by considering only the unique solution for the variable $f(x)$ of the flat system (6.6). Clearly, this yields the desired tree (6.4).

\section{Interpreted Recursive Program Schemes}

We have seen in the previous section that for every guarded recursive program scheme we can find a unique uninterpreted solution. In practice, however, one is more interested in finding interpreted solutions. In the classical treatment of recursive program schemes, this means that a recursive program scheme defining new operation symbols of a signature $\Phi$ from given ones in a signature $\Sigma$ comes together with some $\Sigma$-algebra $A$. An interpreted solution of the recursive program scheme in question is, then, an operation on $A$ for each operation symbol in $\Phi$ such that the formal equations of the RPS become valid identities in $A$.

Of course, in general an algebra $A$ will not admit interpreted solutions. We shall prove in this section that any Elgot algebra $\left(A, a,()^{*}\right)$ as defined in Section 3 admits an interpreted solution of any guarded recursive program scheme. Moreover, if $A$ is a completely iterative algebra, interpreted solutions are unique. We also 
define the notion of a standard interpreted solution and prove that uninterpreted solutions and standard interpreted ones are consistent with one another as expected. This is a fundamental result for algebraic semantics.

We turn to applications after proving our main results. In Subsection 7.1 we study the computation tree semantics of RPSs arising from the computation tree Elgot algebras of Section 3.3 Then, in Subsection 7.2 we prove that in the category CPO our interpreted program scheme solutions agree with the usual denotational semantics obtained by computing least fixed points. Similarly, we show in Subsection 7.3 for the category CMS of complete metric spaces that our solutions are the same as the ones computed using Banach's Fixed Point Theorem. Furthermore, we present new examples of recursive program scheme solutions pertaining to fractal self-similarity. We are not aware of any previous work connecting recursion to implicitly defined sets.

Definition 7.1. Let $(A, a)$ be an $H$-algebra. Let $e: V \longrightarrow T^{H+V}$ be an RPS, and recall the associated monad morphism $\widehat{e}: T^{H+V} \longrightarrow T^{H+V}$ from Lemma 6.9 An interpreted solution of $e$ in $A$ is a morphism $e_{A}^{\ddagger}: V A \longrightarrow A$ such that the following conditions hold:

(i) There is an operation $(-)^{+}$of taking solutions under which $\left(A,\left[a, e_{A}^{\ddagger}\right],(-)^{+}\right)$is an Elgot algebra for $H+V$.

(ii) For $\beta=\widetilde{\left[a, e_{A}^{\ddagger}\right]}$, the diagram below commutes:

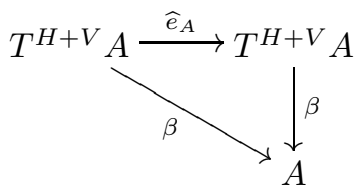

(Recall that this is the solution of $\alpha_{A}^{H+V}: T^{H+V} A \longrightarrow(H+V) T^{H+V} A+A$ in the Elgot algebra $A$ from part (i). Put differently, $\beta$ it is the Eilenberg-Moore algebra structure associated to that Elgot algebra.)

\section{Remark 7.2.}

(i) The subscript $A$ in $e_{A}^{\ddagger}$ is only present to remind us of the codomain $A$. That is, $e_{A}^{\ddagger}$ is not a component of any natural transformation.

(ii) In our leading example where $H=H_{\Sigma}$ and $V=H_{\Phi}$ are signature functors on Set, the commutativity of (7.1) states precisely that the evaluation map $\beta$ arising from an interpreted solution interprets symbols of $\Phi$ in any tree from $T_{\Sigma+\Phi} A$ by operations that satisfy the equations given by the recursive program scheme. More precisely, suppose that $e$ is a recursive program scheme given by formal equations as in (2.5). The interpreted solution $e_{A}^{\ddagger}$ gives for each $n$-ary operation symbol $f$ of the signature $\Phi$ an operation $f_{A}: A^{n} \longrightarrow A$, and together with the given operations for symbols from $\Sigma$ these new operations give an Elgot algebra structure for $\Sigma+\Phi$ on $A$. The commutativity of (7.1) expresses the following property of the associated evaluation map $\beta$ : take any tree $t$ in $T_{\Sigma+\Phi} A$ and second-order substitute every operation symbol from $\Phi$ in $t$ by its right-hand side in the given recursive program scheme to obtain the tree $t^{\prime}$ - this is the result of applying $\widehat{e}_{A}$ to $t$; then the result of evaluating $t^{\prime}$ with $\beta$ in $A$ is $\beta(t)$.

(iii) The requirement that $\left[a, e_{A}^{\ddagger}\right]$ be part of the structure of an Elgot algebra may seem odd at first. However, we need to assume this in order to be able to define $\beta=\widetilde{\left[a, e_{A}^{\ddagger}\right]}$ in (7.1). Furthermore, the requirement has a clear practical advantage: operations defined recursively by means of an interpreted solution of an RPS may be used in subsequent recursive definitions. For example, for the signature functors on Set as in (ii) above the Elgot algebra with structure map $\left[a, e_{A}^{\ddagger}\right]$ has operations for all operation symbols of $\Sigma+\Phi$. Thus, it can be used as an interpretation of givens for any further recursive program scheme with signature $\Sigma+\Phi$ of givens. 
Lemma 7.3. Let $e_{A}^{\ddagger}: V A \longrightarrow A$ be an interpreted solution of an RPS $e$ in an Elgot algebra $\left(A, a,\left({ }_{-}\right)^{*}\right)$.

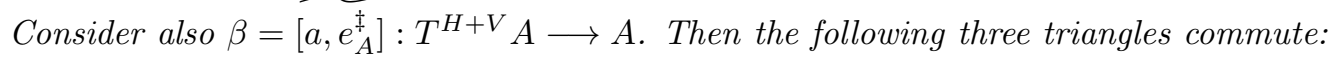

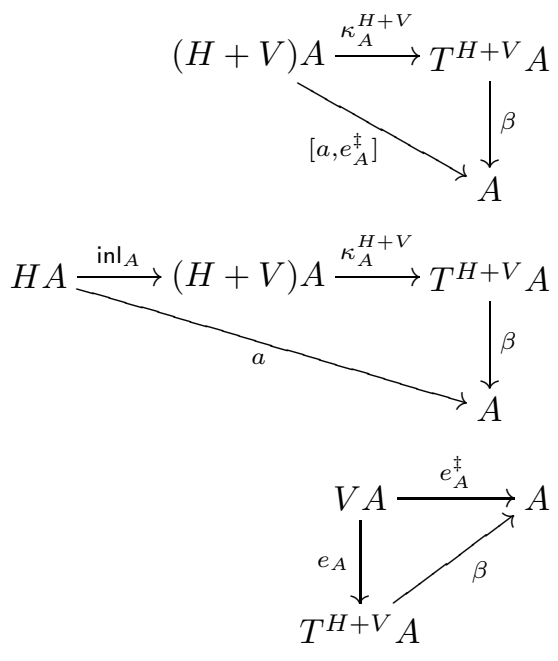

Proof. For (7.2) see Corollary 3.17 and (7.3) follows easily from this. For (7.4), we verify the commutativity of the diagram below, where we write $T$ as a short notation for $T^{H+V}$ and $\lambda$ for $\left[\kappa^{H+V} \varnothing \operatorname{inl}, e\right]: H+V \longrightarrow T$ :

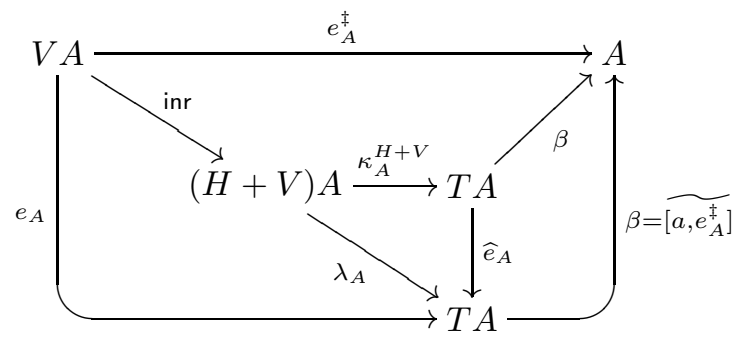

The inner triangle commutes due to the definition of $\widehat{e}$ (see Lemma 6.9), the right-hand one due to (7.1), and the other two parts are clear.

In the previously published version of this paper we incorrectly used (7.4) in the definition of an interpreted solution in lieu of (7.1). The following example shows that the two definitions are not equivalent as we originally claimed.

Example 7.4. Consider the signature $\Sigma$ with a unary operation symbol $F$. The associated signature functor is $H_{\Sigma}=I d$. Take the $\Sigma$-algebra $A=\{0,1\}$ with the unary operation $F_{A}=i d_{A}$. This is a CPO-enrichable algebra in the sense of Example 3.10(ii); thus, $A$ is an Elgot algebra for $H_{\Sigma}$. Notice that $T^{H_{\Sigma}} X=\{F\}^{*} \times X+1$, where the element of 1 stands for the infinite sequence $F^{\omega}=F F F \cdots$. So the associated evaluation map $T_{\Sigma} A \longrightarrow A$ assigns to each $\left(F^{n}, i\right), i=0,1$, the element $i$ and to $F^{\omega}$ the least element 0 of $A$.

Now consider the formal equation $f(x) \approx F(x)$ which defines a guarded RPS $e: V \longrightarrow T^{H_{\Sigma}+V}$, where $V=I d$, as explained in Section 2.3. We verify now that the unary operation $f_{A}=i d_{A}$ is an $V$-algebra structure such that condition (i) in Definition 7.1 holds and such that (7.4) commutes. Observe first that

$$
T^{I d+H_{\Sigma}} X=\{f, F\}^{*} \times X+\{f, F\}^{\omega} .
$$

To see that $\left[F_{A}, f_{A}\right]: A+A \longrightarrow A$ is part of the structure of an Elgot algebra consider the map $\beta$ : $T^{I d+H_{\Sigma}} A \longrightarrow A$ defined by

$$
\begin{aligned}
\beta:(w, i) & \longmapsto i \quad \text { for } w \in\{f, F\}^{*}, \\
v & \longmapsto \begin{cases}1 & \text { if } v=u f^{\omega}, u \in\{f, F\}^{*}, \\
0 & \text { else. }\end{cases}
\end{aligned}
$$

It is not difficult to verify that this map $\beta$ is an Eilenberg-Moore algebra for $T^{I d+H_{\Sigma}}$ (for details see [ABM]). By the first line of the definition we also see that $\beta \varnothing \kappa_{A}^{I d+H_{\Sigma}}=\left[f_{A}, F_{A}\right]$. Now the commutativity of (7.4) expresses precisely that $f_{A}(i)=F_{A}(i)$ holds, which is true since $f_{A}=F_{A}=i d_{A}$. 
However, $f_{A}$ is not an interpreted solution of $e$ in $A$ in the sense of Definition [7.1. The commutativity of (7.1) expresses that the evaluation map $\beta$ is consistent with the second order substitution $\widehat{e}_{A}$ which replaces in any infinite tree from $T^{I d+H_{\Sigma}} A$ any variable symbol from the RPS $e$ by its right-hand side, and so in our example $\widehat{e}_{A}$ replaces every symbol $f$ by a symbol $F$. Thus, we have

$$
\beta\left(f^{\omega}\right)=1 \neq 0=\beta\left(F^{\omega}\right)=\beta \varnothing \widehat{e}_{A}\left(f^{\omega}\right) .
$$

Theorem 7.5. Let $\left(A, a,\left({ }_{-}\right)^{*}\right)$ be an Elgot algebra for $H$, and let $e: V \longrightarrow T^{H+V}$ be a guarded RPS. Then the following hold:

(i) there exists an interpreted solution $e_{A}^{\ddagger}$ of e in $A$,

(ii) if $A$ is a completely iterative algebra, then $e_{A}^{\ddagger}$ is the unique interpreted solution of $e$ in $A$.

Proof. (i) Given an Elgot algebra $\left(A, a,\left({ }_{-}\right)^{*}\right)$ and a guarded recursive program scheme $e: V \longrightarrow T^{H+V}$ consider $\bar{e}: T^{H+V} \longrightarrow H \varnothing T^{H+V}+I d$ from Lemma 6.9. Its component at $A$ yields a flat equation morphism

$$
g \equiv T^{H+V} A \longrightarrow H T^{H+V} A+A,
$$

with respect to $\left(A, a,\left({ }_{-}\right)^{*}\right)$ and we take its solution

$$
\beta \equiv T^{H+V} A \stackrel{g^{*}}{\longrightarrow} A
$$

We will prove that the morphism

$$
e_{A}^{\ddagger} \equiv V A \stackrel{\mathrm{inr}}{\longrightarrow}(H+V) A \stackrel{\kappa_{A}^{H+V}}{\longrightarrow} T^{H+V} A \stackrel{g^{*}}{\longrightarrow} A
$$

is an interpreted solution of $e$ in $A$. We proceed in four steps (a)-(d).

(a) We first check that $\beta$ an evaluation morphism; more precisely, $\beta$ is the structure of an Eilenberg-Moore algebra for $T^{H+V}$. To this end we first establish the equation

$$
\beta=\widetilde{a} ø h_{A},
$$

where $h: T^{H+V} \longrightarrow T^{H}$ is the monad morphism that we obtain from the recursive program scheme $e$, see (6.8). (It is also useful to recall that $h=\overline{\left[\kappa^{H}, e^{\dagger}\right]}$.) Recall that $\widetilde{a}=\left(\alpha_{A}\right)^{*}$, see Theorem [3.15], and that $h_{A}$ is a homomorphism of coalgebras for $H\left({ }_{-}\right)+A$, see Diagram (6.8). Thus, by the Functoriality of $\left({ }^{-}\right)^{*}$, we obtain $g^{*}=\left(\alpha_{A}\right)^{*} \varnothing h_{A}$. By (7.5) and (3.6), this is the desired Equation (7.8). Now since $h: T^{H+V} \longrightarrow T^{H}$ is a monad morphism, and $\widetilde{a}$ is the structure morphism of an Eilenberg-Moore algebra for $T^{H}, \beta=\widetilde{a} ø h_{A}$ is an Eilenberg-Moore algebra for $T^{H+V}$. In fact, this follows from a general fact from category theory, see e. g. Proposition 4.5.9 in $[\mathrm{Bo}$.

(b) We check that the diagram (7.1) commutes for $\beta$ from (7.6). Let us use $T$ as a short notation for $T^{H+V}$ and $T^{\prime}$ for $T^{H}$; we also use $\eta^{\prime}, \tau^{\prime}$, and $\mu^{\prime}$ to denote the natural transformations associated with $T^{\prime}$. Consider the following diagram

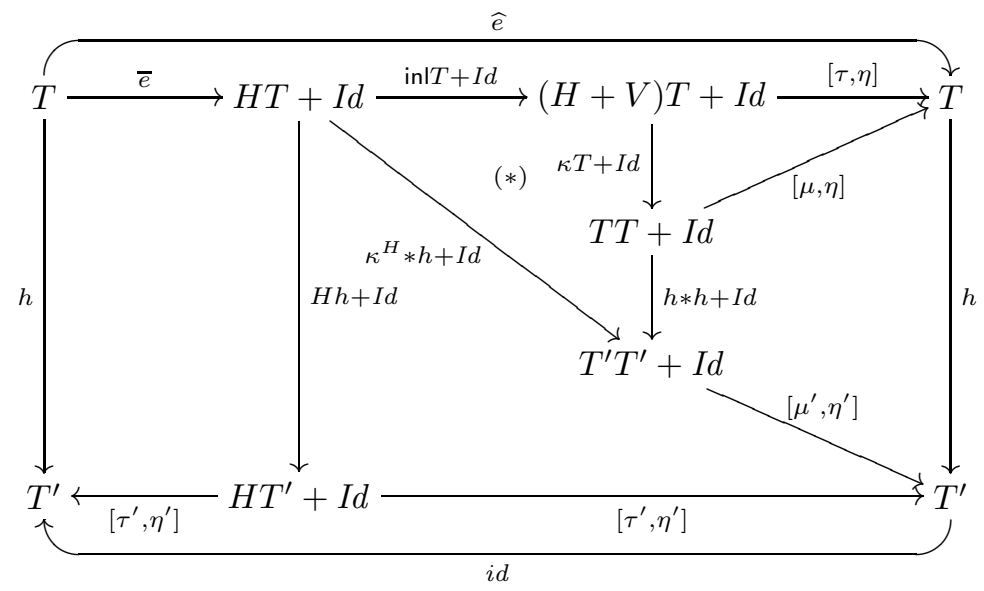

All of its inner parts commute. The lowest part trivially commutes, the upper part is Lemma 6.12 for the left-hand square, see (6.8), and for the right-hand part use that $h$ is a monad morphism. That part $(*)$ commutes follows from commutativity of the left-hand triangle of (6.8) and the double interchange law (2.3). The right-hand coproduct component of the remaining inner part trivially commutes, and the left-hand 
component commutes due to Corollary 3.17 $\mu \varnothing \kappa T=\tau$. Thus, the outer shape of diagram (7.9) commutes. We obtain the equations

$$
\begin{aligned}
\beta \varnothing \widehat{e}_{A} & =\widetilde{a} \varnothing h_{A} \varnothing \widehat{e}_{A} & & (\operatorname{see}(7.8) \\
& =\widetilde{a} \varnothing h_{A} & & (\operatorname{see}(7.9)) \\
& =\beta & & (\operatorname{see}(7.8))
\end{aligned}
$$

(c) We argue that the diagram below commutes:

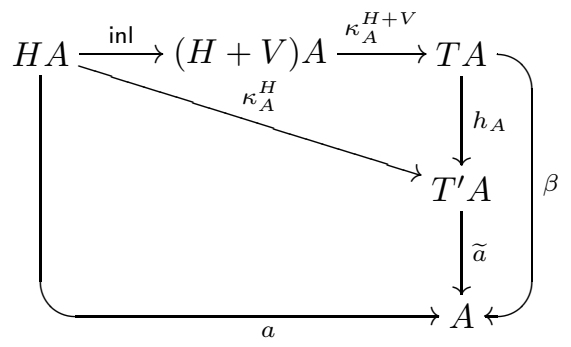

Indeed, the upper triangle commutes by (6.8), the right-hand part commutes by (7.8) and for the commutativity of the lower part see Corollary 3.17. Thus, the outward shape of the diagram commutes.

(d) We are ready to prove that $e_{A}^{\ddagger}$ as defined in (7.7) is an interpreted solution of $e$ in $A$. From the diagram in (c) and the definition of $e_{A}^{\ddagger}$ we see that the equation

$$
\beta \varnothing \kappa_{A}^{H+V}=\left[a, e_{A}^{\ddagger}\right]
$$

holds. Recall that by (a) above, $\beta$ is an evaluation morphism. This fact, together with the equation just above, imply that $\left[a, e_{A}^{\ddagger}\right]$ is the structure morphism of an Elgot algebra on $A$ (cf. Theorem [3.16). This establishes item (i) in Definition 7.1, and item (ii) was established in (b) above. This completes the proof of part (i).

(ii) Let $\left(A, a,\left({ }_{-}\right)^{*}\right)$ be a cia. We show that the solution $e_{A}^{\ddagger}$ defined in (i) is unique. We will now verify that any evaluation morphism $\beta: T^{H+V} A \longrightarrow A$ for which diagram (7.1) commutes is a solution of $g$ as defined in (7.5). To this end consider the following diagram, where we write $T=T^{H+V}$ for short once more:

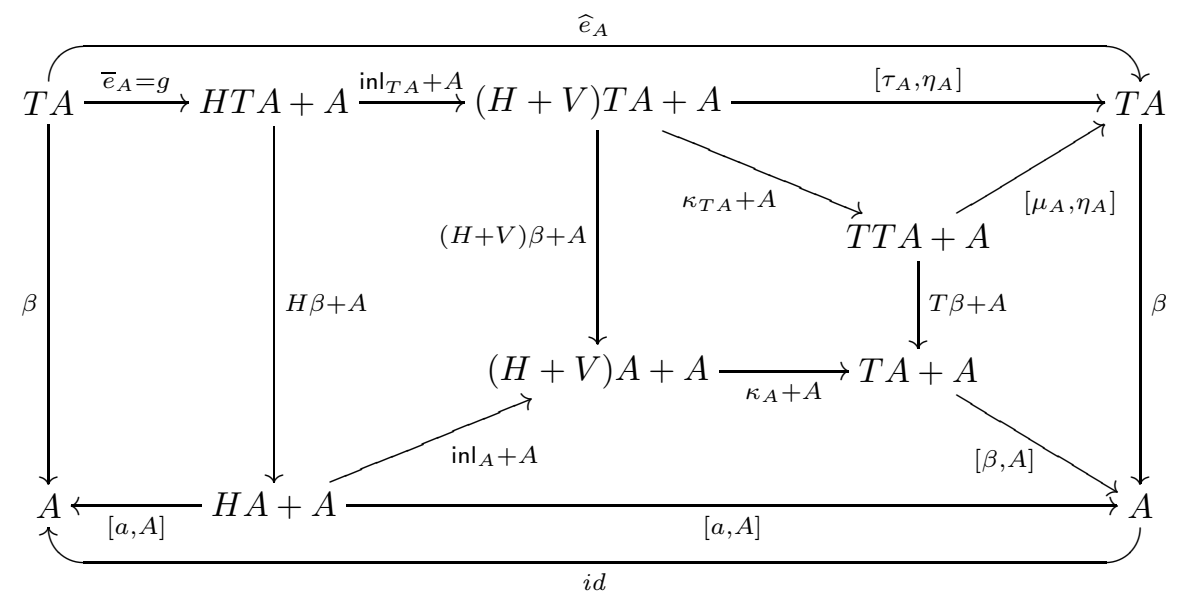

Its outer shape commutes due to (7.1), the right-hand part since $\beta$ is an Eilenberg-Moore algebra structure, the upper-right triangle follows from Corollary 3.17, and the lower right-hand part follows from (7.3). Thus, since all other parts are obviously commutative, the left-hand inner square commutes. But this shows that $\beta$ is a solution of $g$. By the uniqueness of solutions, we have $\beta=g^{*}$.

To complete our proof, we appeal to the first part of Lemma 7.3 to see that the interpreted solutions are always determined by their evaluation morphisms $\beta$. This means that interpreted solutions in cias are unique.

Definition 7.6. For any guarded RPS $e$ and any Elgot algebra $\left(A, a,(\text { - })^{*}\right)$, let $e_{A}^{\ddagger}$ be the interpreted solution obtained from the proof of Theorem[7.5 as stated below

$$
e_{A}^{\ddagger} \equiv V A \stackrel{\mathrm{inr}}{\longrightarrow}(H+V) A \stackrel{\kappa_{A}^{H+V}}{\longrightarrow} T^{H+V} A \stackrel{g^{*}}{\longrightarrow} A,
$$


where $g$ is the flat equation morphism of (7.5). We call this the standard interpreted solution of $e$ in $A$.

It follows from the proof of Theorem 7.5 that uninterpreted solutions correspond to certain interpreted ones in a canonical way. We now make this precise and thereby prove what could be called "Fundamental Theorem of Algebraic Semantics".

Theorem 7.7. Let $\left(A, a,\left({ }_{-}\right)^{*}\right)$ be an Elgot algebra and consider its evaluation morphism $\widetilde{a}: T^{H} A \longrightarrow A$. Let e be any guarded recursive program scheme, let $e_{A}^{\ddagger}: V A \longrightarrow A$ be the standard interpreted solution of $e$ in $A$, and let $e^{\dagger}: V \longrightarrow T^{H}$ be the (uninterpreted) solution of Theorem 6.5. Then the triangle

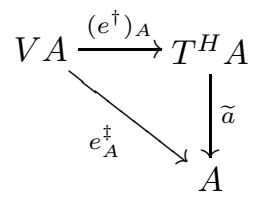

commutes.

Furthermore, the standard interpreted solution $e_{A}^{\ddagger}$ is uniquely determined by the commutativity of the above triangle.

Remark 7.8. Notice that $\left(e^{\dagger}\right)_{A}$ is the component at $A$ of the natural transformation $e^{\dagger}$. And once again, $e_{A}^{\ddagger}$ is not the component at $A$ of any natural transformation but merely a morphism from $V A$ to $A$.

Proof. In fact, we have the commutative diagram

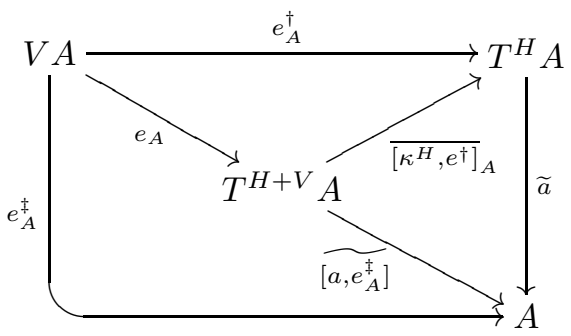

The lower left part commutes by Lemma 7.3, see (7.4); the top triangle is the definition of an uninterpreted solution, see (6.2); and the triangle on the right commutes by $\left(\overline{7.8)}\right.$ since $h=\overline{\left[\kappa^{H}, e^{\dagger}\right]}$ and $\beta=\widetilde{\left[a, e_{A}^{\ddagger}\right]}$. Hence (7.10) follows immediately.

It is obvious that $e_{A}^{\ddagger}$ is uniquely determined by the commutativity of the triangle (7.10), as neither $\widetilde{a}$ nor $e^{\dagger}$ depend on $e_{A}^{\ddagger}$.

7.1. Interpreted Solutions in Computation Tree Elgot Algebras. In this section, we work through some details concerning the factorial example of Equation (1.3), namely

$$
f(n) \approx \operatorname{ifzero}(n, \text { one, } f(\operatorname{pred}(n) * n))
$$

and studied further in Example 6.4(i). Recall that we work with the signature $\Sigma$ containing a constant one, one unary symbol pred, a binary symbol $*$ and a ternary one ifzero. Let $H_{\Sigma}$ be the associated endofunctor on Set. Let $\uparrow$ be an object which is not a natural number, and let $\mathbb{N}_{\uparrow}$ be the $H_{\Sigma}$-algebra with carrier $\{0,1,2, \ldots\} \cup\{\uparrow\}$ and whose operations are the strict extensions of the standard operations. (For example $\uparrow * 3=\uparrow$ in this algebra.)

We shall use the computation tree Elgot algebra structure $\left(\mathbb{N}_{\uparrow}, a,\left({ }_{-}\right)^{*}\right)$ from Section 3.3. That structure used a particular element of the carrier set in connection with the conditional ifzero, and in our structure we take 0. Before looking at the interpreted solution to the factorial RPS, it might be useful to spell out the associated evaluation morphism $\widetilde{a}: T^{H_{\Sigma}} \mathbb{N}_{\uparrow} \longrightarrow \mathbb{N}_{\uparrow}$. Let $t$ be a finite or infinite $\Sigma$-tree over $\mathbb{N}_{\uparrow}$; so the leaves 
of $t$ might be labelled with natural number or $\uparrow$, but not with formal variables. Here is a pertinent example:

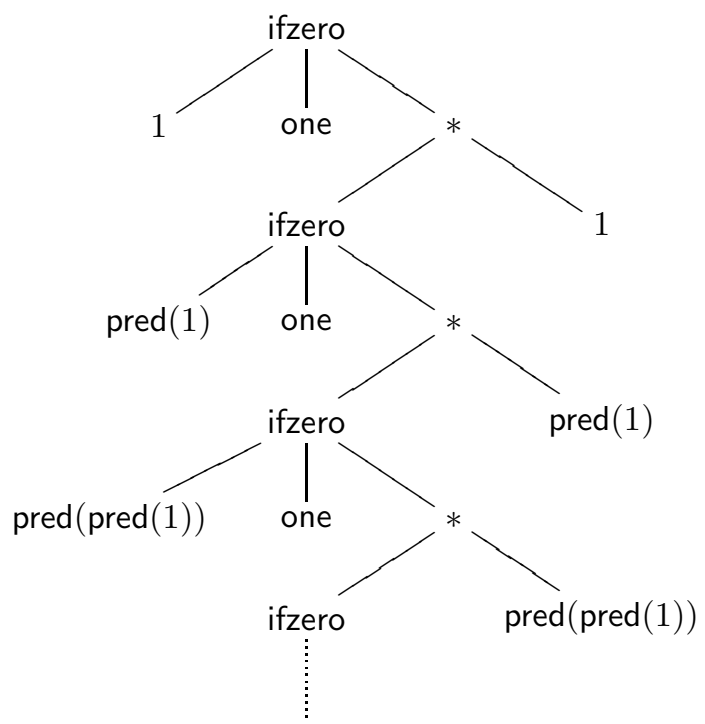

We got this by taking the uninterpreted solution of our RPS, as depicted in (6.4), and then substituting the number 1 for the formal variable $n$. Note that the nodes labelled ifzero have three children. Let $t$ again be a $\Sigma$-tree over $\mathbb{N}_{\uparrow}$. Here is how we define $\widetilde{a}(t)$. We look for a finite subtree $u$ of $t$ with the property that if a node belongs to $u$ and is labelled by a function symbol other than ifzero, then all its children belong to $u$ as well; and if the node is labeled by ifzero, then either the first and second children belong to $u$, or else the first and third children do. For such a finite subtree $u$, we can evaluate the nodes in a bottom-up fashion using the $H_{\Sigma}$-algebra structure. We require that for a conditional node $x$, the first child evaluates to 0 (from our Elgot algebra structure) iff the second child is in $u$. If such a finite $u$ exists, then we can read off an element of $\mathbb{N}_{\uparrow}$. This element is $\widetilde{a}(t)$. If no finite $u$ exists, we set $\widetilde{a}(t)=\uparrow$. Returning to our example above, the finite subtree would be

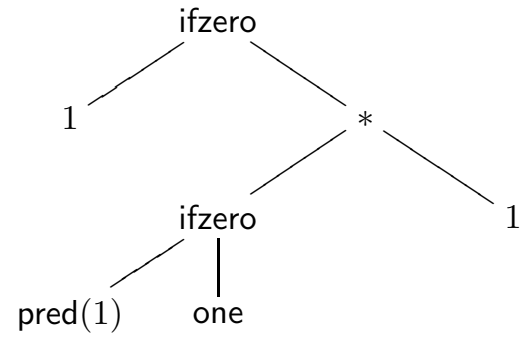

And for our example tree $t, \widetilde{a}(t)=1$.

We are now in a position to discuss the interpreted solution of our RPS. Recall that the signature $\Phi$ of recursively defined symbols contains only the unary symbol $f$. The corresponding signature functor is $H_{\Phi}$, and $H_{\Phi}\left(\mathbb{N}_{\uparrow}\right)$ is the set $\{f(0), f(1), \ldots\} \cup\{f(\uparrow)\}$. The RPS itself is a natural transformation $e: H_{\Phi} \longrightarrow$ $T^{H_{\Sigma}+H_{\Phi}}$. The uninterpreted solution is the natural transformation $e^{\dagger}: H_{\Phi} \longrightarrow T^{H_{\Sigma}}$ corresponding to the tree shown in (6.4). We are concerned here with the interpreted solution $e_{\mathbb{N}_{\uparrow}}^{\ddagger}: H_{\Phi}\left(\mathbb{N}_{\uparrow}\right) \longrightarrow \mathbb{N}_{\uparrow}$ of our RPS. In light of the Fundamental Theorem $\left[7.7\right.$ this is $\widetilde{a} \varnothing\left(e^{\dagger}\right)_{\mathbb{N}_{\uparrow}}$. We show by an easy induction on $n \in N$ that this interpreted solution takes $f(n)$ to $n$ !, and that it takes $f(\uparrow)$ to $\uparrow$.

We could also establish this same result directly, without Theorem 7.7 To do this, we follow the proof of Theorem 7.5. We turn our RPS $e$ into a related natural transformation $\bar{e}: T^{H_{\Sigma}+H_{\Phi}} \longrightarrow H_{\Sigma} T^{H_{\Sigma}+H_{\Phi}}+I d$. Then $\bar{e}_{\mathbb{N}_{\uparrow}}$ is a flat equation morphism in the Elgot algebra $\mathbb{N}_{\uparrow}$, and its solution is the interpreted solution of 
our RPS. Here is a fragment of $\bar{e}_{\mathbb{N}_{\uparrow}}$ :

$$
\begin{aligned}
& \underline{f(0)} \approx \text { ifzero }(\underline{0}, \text { one }, \underline{f(\operatorname{pred}(0)) * 0)} \\
& \underline{f(1)} \approx \text { ifzero }(\underline{1}, \text { one }, \underline{f(\operatorname{pred}(1)) * 1)} \\
& \underline{f(\operatorname{pred}(1))} \approx \text { ifzero }(\operatorname{pred}(1), \underline{\text { one }}, \underline{f(\operatorname{pred}(\operatorname{pred}(1))) * \operatorname{pred}(1))} \\
& \underline{f(\operatorname{pred}(1)) * 1} \approx \underline{\text { ifzero }(\operatorname{pred}(1), \text { one }, f(\operatorname{pred}(\operatorname{pred}(1))) * \operatorname{pred}(1))} * \underline{1} \\
& \underline{\operatorname{pred}(1)} \approx \operatorname{pred}(\underline{1}) \\
& \text { one } \approx 1
\end{aligned}
$$

One can see that for each natural number $n$, the solution to this flat equation morphism assigns to $\underline{f(n)}$ the number $n$ !.

7.2. Interpreted Solutions in CPO. We shall show in this subsection that if we have $\mathcal{A}=\mathrm{CPO}$ as our base category, then interpreted solutions of guarded RPSs $e$ in an Elgot algebra $\left(A, a,(\text { ـ })^{*}\right)$ are given as least fixed points of a continuous operator on a function space. In this way we recover denotational semantics from our categorical interpreted semantics of recursive program schemes.

Example 7.9. We study the RPS of Equation (1.3) as formalized in Example 6.4(i). As we know, the intended interpreted solution is the factorial function on the natural numbers $\mathbb{N}$.

This time we turn the natural numbers into an object of CPO so as to obtain a suitable Elgot algebra in which we can find an interpreted solution of (1.3). Let $\mathbb{N}_{\perp}$ be the flat cpo obtained from the discretely ordered $\mathbb{N}$ by adding a bottom element $\perp$, so $x \leq y$ iff $x=\perp$ or $x=y$. We equip $\mathbb{N}_{\perp}$ with the strict operations one $\mathbb{N}_{\perp}$, $\operatorname{pred}_{\mathbb{N}_{\perp}}$, and $*_{\mathbb{N}_{\perp}}$. Being strict, they are hence continuous. In addition, we use the continuous function

$$
\text { ifzero }_{\mathbb{N}_{\perp}}(n, x, y)= \begin{cases}\perp & \text { if } n=\perp \\ x & \text { if } n=0 \\ y & \text { else }\end{cases}
$$

Indeed, this is what we saw in (3.4) for the computation tree semantics, except we write $\perp$ for $\uparrow$. Hence we have a continuous $\Sigma$-algebra with $\perp$. Therefore $\mathbb{N}_{\perp}$ is an Elgot algebra for $H_{\Sigma}$ : Set $\longrightarrow$ Set, see Example 3.10(ii).

The standard interpreted solution $e_{\mathbb{N}_{\perp}}^{\ddagger}: H_{\Phi} \mathbb{N}_{\perp} \longrightarrow \mathbb{N}_{\perp}$ will certainly be some function or other on $\mathbb{N}_{\perp}$. But how do we know that this function is the desired factorial function? Usually one would simply regard the RPS (1.3) itself as a continuous function $R$ on $\mathrm{CPO}\left(\mathbb{N}_{\perp}, \mathbb{N}_{\perp}\right)$ acting as

$$
f\left({ }_{-}\right) \longmapsto \text { ifzero }_{\mathbb{N}_{\perp}}\left({ }_{-}, 1, f\left(\operatorname{pred}_{\mathbb{N}_{\perp}}\left(\text { - }_{-}\right) *_{\mathbb{N}_{\perp}}-\right),{ }_{-}\right) ;
$$

Hence $R$ is the operator described in (1.6) in the introduction. That means that we interpret all the operation symbols of $\Sigma$ in the algebra $\mathbb{N}_{\perp}$. The usual denotational semantics assigns to the formal equation of (1.3) with the interpretation in $\mathbb{N}_{\perp}$ the least fixed point of $R$. Clearly this yields the desired factorial function. And it is not difficult to work out that the least fixed point of $R$ coincides with the standard interpreted solution $e_{\mathbb{N}_{\perp}}^{\ddagger}$ obtained from Theorem 7.5. We shall do this shortly in greater generality.

In general, any recursive program scheme can be turned into a continuous operator $R$ on the function space $\operatorname{CPO}(V A, A)$. Theorem 7.11 below shows that the least fixed point of $R$ is the same as the interpreted solution obtained from Theorem 7.5

We assume throughout this subsection that $H, V$ and $H+V$ are locally continuous (and, as always, iteratable) endofunctors of CPO. We also assume that the free completely iterative monad $T^{H+V}$ is locally continuous. We consider a fixed guarded RPS $e: V \longrightarrow T^{H+V}$, and an $H$-algebra $(A, a)$ with a least element $\perp$. By Example $3.10(\mathrm{i})$, we know that this carries the structure of an Elgot algebra $\left(A, a,(\mathrm{z})^{*}\right)$, where $\left(\mathrm{A}^{*}\right.$ assigns to every flat equation morphism a least solution. As before, we will use the notation $\widetilde{a}: T^{H} A \longrightarrow A$ for the induced evaluation morphism.

For any continuous map $t: V A \longrightarrow A$, we have an Elgot algebra on $A$ for the functor $H+V$; the structure is $[a, t]$, and again the solution operation takes least solutions to flat equation morphisms. Due to Corollary 3.17, we have

$$
\widetilde{[a, t]} \phi \kappa_{A}^{H+V}=[a, t] .
$$

Before we prove the main result of this subsection we need to recall a result from [ABM which states that the operation of taking least solutions in any continuous algebra $[a, t]:(H+V) A \longrightarrow A$ extends to non-flat 
equation morphisms as in Section 4. Moreover, those least solutions are properly connected to the unique solutions in the free completely iterative monad on $H+V$.

Let us write $T=T^{H+V}$ and $\left.\gamma=\widetilde{[a, t}\right]$ for short. Now consider any (non-flat) guarded equation morphism $d: X \longrightarrow T(X+A)$ (see Definition 4.5). The least solution of $d$ in the algebra $A$ is the join $d^{*}: X \longrightarrow A$ of the following $\omega$-chain $\left(d_{i}^{*}\right)$ in $\mathrm{CPO}(X, A): d_{0}^{*}=$ const $_{\perp}$ and given $d_{i}^{*}: X \longrightarrow A$, we define $d_{i+1}^{*}$ by the commutativity of the following square

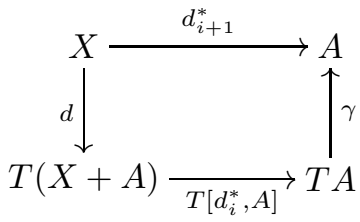

Notice that we use here that $T$ is locally continuous in order to see that $\left(d_{i}^{*}\right)$ is an $\omega$-chain. Furthermore, observe that $d^{*}$ is a solution of $d$ in $A$ in the sense that the equation $d^{*}=\gamma \varnothing T\left[d^{*}, A\right] \varnothing d$ holds.

Theorem 7.10. (see $[\mathrm{ABM}]$ ) The least solution of $d$ in $A$ is the morphism $\gamma \varnothing d^{\dagger}$, where $d^{\dagger}: X \longrightarrow T A$ is the unique solution of $d$ w.r.t. the completely iterative monad $T$; in symbols:

$$
d^{*} \equiv X \stackrel{d^{\dagger}}{\longrightarrow} T A \stackrel{\gamma}{\longrightarrow} A .
$$

We are now prepared to prove the main result of the current subsection.

Theorem 7.11. The following function $R$ on $\operatorname{CPO}(V A, A)$

$$
f \longmapsto V A \stackrel{e_{A}}{\longrightarrow} T^{H+V} A \stackrel{\widetilde{[a, f]}}{\longrightarrow} A
$$

is continuous. Its least fixed point is the standard interpreted solution $e_{A}^{\ddagger}: V A \longrightarrow A$ of Theorem 7.5.

Proof. (i) To see the continuity of $R$ is suffices to prove that the function

$$
\widetilde{(-)}: \mathrm{CPO}(H A, A) \longrightarrow \mathrm{CPO}\left(T^{H} A, A\right)
$$

is continuous. Let us write $T$ for $T^{H}$. Recall that for any continuous map $a: H A \longrightarrow A$, the evaluation morphism $\widetilde{a}$ is the least solution of the flat equation morphism $\alpha_{A}: T A \longrightarrow H T A+A$. This means that $\widetilde{a}$ is the least fixed point of the continuous function

$$
F(a,-): \mathrm{CPO}(T A, A) \longrightarrow \mathrm{CPO}(T A, A) \quad f \longmapsto[a, A] \varnothing(H f+A) \varnothing \alpha_{A} .
$$

Observe that $F$ is continuous in the first argument $a$, and so $F$ is a continuous function on the product $\mathrm{CPO}(H A, A) \times \mathrm{CPO}(T A, A)$. It follows from standard arguments that taking the least fixed point in the second argument yields a continuous map $\mathrm{CPO}(H A, A) \longrightarrow \mathrm{CPO}(T A, A)$. But this is precisely the desired one $\widetilde{(-)}$.

(ii) We prove that $e_{A}^{\ddagger}$ is the least fixed point of $R$. Notice that the least fixed point of $R$ is the join $t$ of the following increasing chain in $\mathrm{CPO}(V A, A)$ :

$$
t_{0}=\text { const }_{\perp}: V A \longrightarrow A, \quad t_{i+1} \equiv V A \stackrel{e_{A}}{\longrightarrow} T^{H+V} A \stackrel{\widetilde{\left[a, t_{i}\right]}}{\longrightarrow} A, \quad \text { for } i \geq 0 .
$$

Recall that the standard interpreted solution $e_{A}^{\ddagger}$ is defined by the equation $e_{A}^{\ddagger}=\beta \varnothing \kappa_{A}^{H+V} \varnothing \mathrm{inr}$ (see Definition (7.6), where $\beta=g^{*}$ is the least solution of the flat equation morphism $g$ from (7.5), which is obtained from the component at $A$ of the $\mathcal{H}$-coalgebra $\bar{e}$, see Lemma 6.9 and Theorem 7.5. Observe that by Lemma 7.3. $e_{A}^{\ddagger}$ is a fixed point of $R$ (see (7.4) ). Thus, we have $t \sqsubseteq e_{A}^{\ddagger}$. To show the reverse inequality we will prove in (iib) below that $\beta \sqsubseteq \widetilde{[a, t]}$ holds. Then we have

$$
e_{A}^{\ddagger}=\beta \varnothing \kappa_{A}^{H+V} \varnothing \mathrm{inr} \sqsubseteq \widetilde{[a, t]} \varnothing \kappa_{A}^{H+V} \varnothing \mathrm{inr}=t,
$$

where the last equality follows from (7.11).

Let us write $T$ for $T^{H+V}, \gamma$ as a short notation for $\widetilde{[a, t]}$, and we also write $\lambda=\left[\kappa^{H+V} \varnothing\right.$ inl, $\left.e\right]$.

(iia) We will now prove that the following inequality

$$
\gamma \varnothing \widehat{e}_{A} \sqsubseteq \gamma \quad \text { holds in } \mathrm{CPO}(T A, A),
$$

where $\widehat{e}: T \longrightarrow T$ is the monad morphism from Lemma 6.9. We use that the domain $T A$ is a coproduct of $(H+V) T A$ and $A$, and we verify (7.14) componentwise. For the right-hand component we extend both 
sides of the inequality by the colimit injection $\eta_{A}^{H+V}: A \longrightarrow T A$, and then we use the unit law of the monad morphism $\widehat{e}: T \longrightarrow T$ :

$$
\gamma \emptyset \widehat{e}_{A} \varnothing \eta_{A}^{H+V}=\gamma \varnothing \eta_{A}^{H+V} .
$$

For the left-hand component of the desired inequality we extend by the coproduct injection $\tau_{A}^{H+V}:(H+$ $V) T A \longrightarrow T A$, and so we need to establish the inequality

$$
\gamma \varnothing \widehat{e}_{A} \varnothing \tau_{A}^{H+V} \sqsubseteq \gamma \varnothing \tau_{A}^{H+V} \quad \text { in } \mathrm{CPO}((H+V) T A, A) .
$$

Recall from AAMV] (see the proof of Theorem 4.14) that the monad morphism $\widehat{e}: T \longrightarrow T$ can be obtained as follows. The component $\widehat{e}_{Y}$ for any object $Y$ is

$$
\left[\left(\lambda * \alpha^{H+V}\right)_{Y}^{\dagger}, \eta_{Y}^{H+V}\right]: T Y=(H+V) T Y+Y \longrightarrow T Y
$$

where we consider the component at $Y$ of the natural transformation

$$
\lambda * \alpha^{H+V}:(H+V) T \longrightarrow T((H+V) T+I d)
$$

to be a guarded equation morphism. Let us write $d=\left(\lambda * \alpha^{H+V}\right)_{A}$ for short. Note that $d: X \longrightarrow T(X+A)$, with $X=(H+V) T A$.

Then, the left-hand side of our desired inequality (7.15) is $\gamma \varnothing d^{\dagger}$, and, by Theorem[7.10, we know that this is the least solution $d^{*}$ of the equation morphism $d$ in $A$. So in order to establish inequality (7.15) above we prove by induction on $i$ that

$$
d_{i}^{*} \sqsubseteq \gamma \varnothing \tau_{A}^{H+V} \quad \text { holds in } \mathrm{CPO}((H+V) T A, A) \text {, for all } i \in \mathbb{N} .
$$

The base case is clear. For the induction step we consider the diagram below (we write $X=(H+V) T A$ for short):

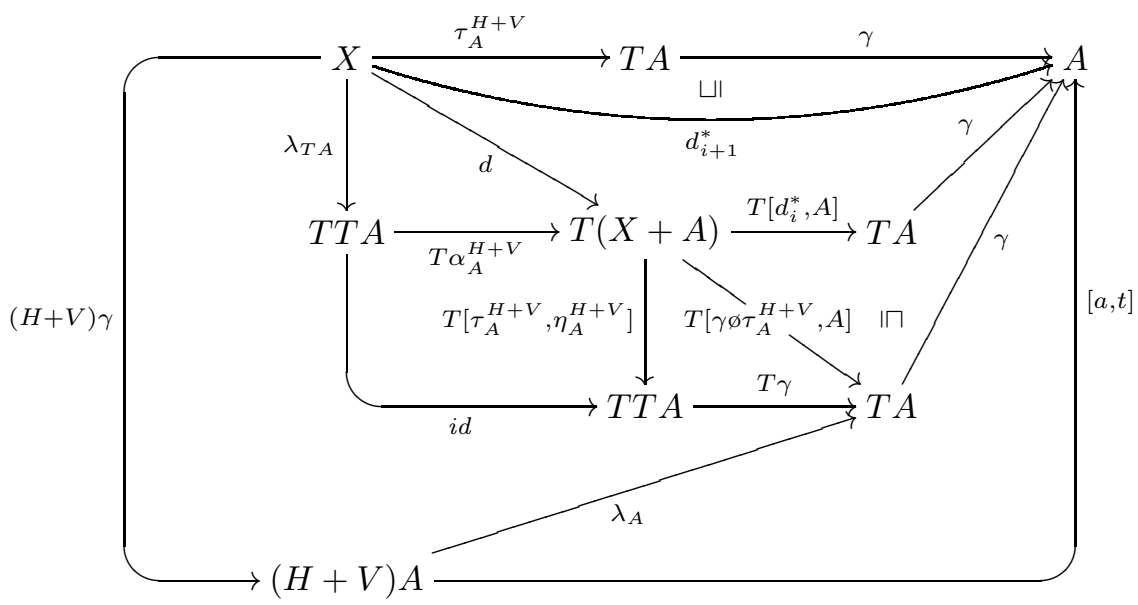

We will verify that the upper inequality holds as indicated. In fact, the part below that inequality commutes by the definition of $d_{i+1}^{*}$ (see Diagram (7.12)); the left-most part commutes by the naturality of $\lambda$; the upper left triangle commutes by the definition of $d$; the part below that triangle commutes since $[\tau, \eta]$ is the inverse of $\alpha$ (see Theorem 3.15(iii)). For the inner triangle remove $T$ and consider the coproduct components separately: the left-hand component trivially commutes, and for the right-hand one use the unit law $\gamma \varnothing \eta_{A}^{H+V}=i d$. The inner inequality holds by the induction hypothesis $d_{i}^{*} \sqsubseteq \gamma \phi \tau_{A}^{H+V}$ and by the fact that copairing, application of $T$ and composition with $\gamma$ on the left are all continuous whence monotone operations. (Note that we use the assumption that $T$ be locally continuous here.) Recall that $\gamma=\widetilde{[a, t}]: T A \longrightarrow A$ is a homomorphism of Elgot algebras, see Theorem 3.15. Hence, $\gamma$ is an homomorphism of algebras for $H+V$ by Proposition 3.7 Thus, the outer shape commutes. Finally we verify the lower right-hand part componentwise: that the left-hand component with domain $H A$ commutes is seen by the following computation

$$
\begin{aligned}
\gamma \emptyset \lambda_{A} \varnothing \mathrm{inl} & =\gamma \varnothing \kappa_{A}^{H+V} \varnothing \mathrm{inl} & & \text { (by the definition of } \lambda) \\
& =[a, t] \varnothing \kappa_{A}^{H+V} \varnothing \mathrm{inl} & & \text { (by the definition of } \gamma) \\
& =[a, t] \varnothing \mathrm{inl} & & \text { (by Equation (7.11)) } \\
& =a, & &
\end{aligned}
$$

and the right-hand component with domain $V A$ is the equation $\gamma \varnothing e_{A}=t$ which holds since $t$ is a fixed point of $R$. This completes the proof of the inequality (7.14). 
(iib) We are ready to establish the inequality $\beta \sqsubseteq \widetilde{[a, t]}$. To this end observe that by Example $\underline{3.10}$ (i), the least solution $\beta$ of $g$ is the join of the chain

$$
\beta_{0}=\text { const }_{\perp}, \quad \beta_{i+1}=[a, A] \varnothing H\left(\beta_{i}+A\right) \varnothing g, \quad \text { for } i \geq 0 .
$$

So in order to establish the desired inequality we prove by induction on $i$ the inequalities

$$
\beta_{i} \sqsubseteq \widetilde{[a, t]}, \quad i \in \mathbb{N} .
$$

The base case is clear. For the induction step we consider the diagram below:

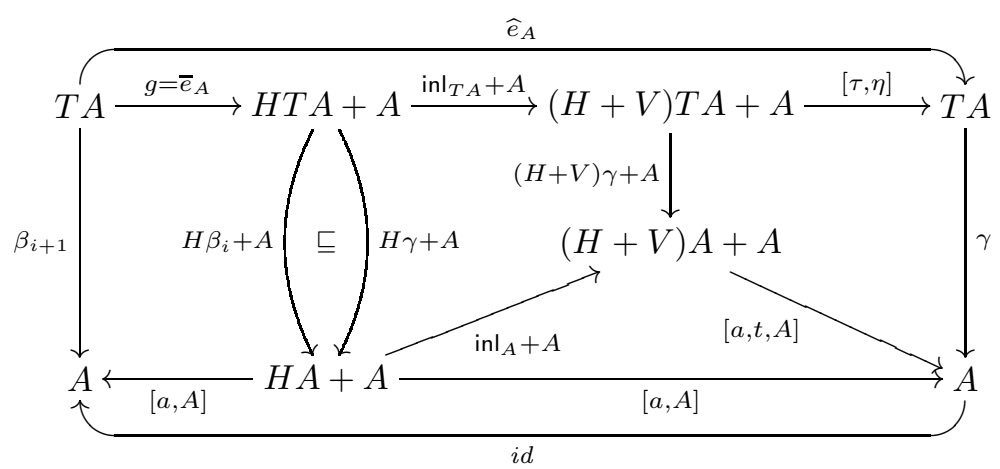

Its upper part commutes due to Lemma 6.12, the left-hand part is the definition of $\beta_{i+1}$, and the inequality follows from the induction hypothesis. For the right-hand part use that $\gamma$ is an algebra homomorphism. Finally, the remaining parts of the above diagram clearly commute. Thus we obtain the inequality $\beta_{i+1} \sqsubseteq$ $\gamma \emptyset \widehat{e}_{A}$, and the desired inequality follows from this by (7.14). This completes the proof.

Remark 7.12. The result of Theorem 7.11 furnishes a concrete formula

$$
e_{A}^{\ddagger}=\bigsqcup_{n<\omega} e_{n}^{\ddagger}
$$

for the interpreted solution of the guarded RPS $e$ in the continuous algebra $A$. In fact, the least fixed point of $R$ is the join of the ascending chain

$$
\perp \sqsubseteq R(\perp) \sqsubseteq R^{2}(\perp) \sqsubseteq \cdots
$$

where $\perp=$ const $_{\perp}$ is the least element of $\operatorname{CPO}(V A, A)$. Thus, with $e_{0}^{\ddagger}=$ const $_{\perp}$ and

$$
e_{n+1}^{\ddagger} \equiv V A \stackrel{e_{A}}{\longrightarrow} T^{H+V} A \stackrel{\widetilde{\left[a, e_{n}^{\ddagger}\right]}}{\longrightarrow} A
$$

we obtain the above formula for $e_{A}^{\ddagger}$.

Remark 7.13. Suppose that $H, V$ and $H+V$ are iteratable endofunctors of Set, which have locally continuous liftings $H^{\prime}$ and $V^{\prime}$ to CPO. Then from Example 2.8 we have a commutative square

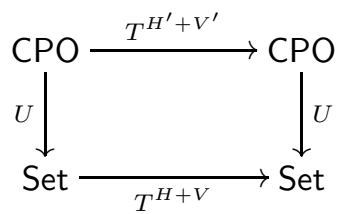

In addition, we now show that $T^{H^{\prime}+V^{\prime}}$ is locally continuous. In fact, for each CPO $X, T^{H^{\prime}+V^{\prime}} X$ is obtained as a limit of an ordinal indexed op-chain of the cpos $T_{\beta}$ (see Example 2.8). Hence, one readily proves that for any continuous map $f: X \longrightarrow Y$ the continuous map $T^{H+V} f$ is obtained by using the universal property of that limit. It follows from standard arguments that the assignment $f \longmapsto T^{H+V} f$ is a continous map on $\mathrm{CPO}(X, Y)$ (see $\mathrm{ABM}$ for details).

Assume that the guarded RPS $e: V \longrightarrow T^{H+V}$ has a lifting $e^{\prime}: V^{\prime} \longrightarrow T^{H^{\prime}+V^{\prime}}$. This is a natural transformation $e^{\prime}$ such that $U e^{\prime}=e U$. Now consider any CPO-enrichable $H$-algebra $(A, a)$ as an Elgot algebra, see Example 3.10(ii). Then we can apply Theorem 7.11 to obtain the standard interpreted solution $e_{A}^{\ddagger}$ of $e$ in the algebra $A$ as a least fixed point of the above function $R$ of (7.13).

\section{Example 7.14.}


(i) Suppose we have signatures $\Sigma$ and $\Phi$. Then the signature functors $H_{\Sigma}$ and $H_{\Phi}$ have locally continuous liftings $H_{\Sigma}^{\prime}$ and $H_{\Phi}^{\prime}$. Since the lifting of $H_{\Sigma}+H_{\Phi}$ is a lifting of $H_{\Sigma+\Phi}$ we know that $T^{H_{\Sigma}^{\prime}+H_{\Phi}^{\prime}}$ assigns to any cpo $X$ the algebra $T_{\Sigma+\Phi} X$ with the cpo structure induced by $X$, see Example 2.8 More precisely, to compare a tree $t$ to a tree $s$ replace all leaves labelled by a variable from $X$ by a leaf labelled by some extra symbol $\star$ to obtain relabelled trees $t^{\prime}$ and $s^{\prime}$. Then $t \sqsubseteq s$ holds in $T_{\Sigma+\Phi} X$ iff $t^{\prime}$ and $s^{\prime}$ are isomorphic as labelled trees, and for any leaf of $t$ labelled by a variable $x$ the corresponding leaf in $s$ is labelled by a variable $y$ with $x \sqsubseteq y$ in $X$.

Now consider any system as in (2.5) which is in Greibach normal form, and form the associated guarded RPS $e: H_{\Phi} \longrightarrow T_{\Sigma+\Phi}$. Then $e$ has a lifting $e^{\prime}: H_{\Phi}^{\prime} \longrightarrow T^{H_{\Sigma}^{\prime}+H_{\Phi}^{\prime}}$. In fact, for any cpo $X$ the component $e_{X}^{\prime}=e_{X}: H_{\Phi} X \longrightarrow T_{\Sigma+\Phi} X$ is a continuous map since the order in $H_{\Phi} X$ is given similarly as for $T_{\Sigma+\Phi} X$ on the level of variables only: $(f, \vec{x}) \sqsubseteq(g, \vec{y})$ holds for elements of $H_{\Phi} X$ if $f=g \in \Phi_{n}$ and $x_{i} \sqsubseteq y_{i}, i=1, \ldots, n$, hold in $X$.

Let $(A, a)$ be a CPO-enrichable $H_{\Sigma}$-algebra; i. e., a continuous $\Sigma$-algebra with a least element $\perp$. We wish to consider the continuous function $R$ on $\mathrm{CPO}\left(H_{\Phi} A, A\right)$ which assigns to any continuous algebra structure $\varphi: H_{\Phi} A \longrightarrow A$ the algebra structure $R(\varphi)=\widetilde{[a, \varphi]} \phi e_{A}^{\prime}$. The structure $R(\varphi)$ : $H_{\Phi} A \longrightarrow A$ gives to each $n$-ary operation symbol $f$ of $\Phi$ the operation $t_{A}^{f}: A^{n} \longrightarrow A$ which is obtained as follows: take the term $t^{f}$ provided by the right-hand side of $f$ in our given RPS, then interpret all operation symbols of $\Sigma$ in $t^{f}$ according to the given algebraic structure $a$ and all operation symbols of $\Phi$ according to $\varphi$; the action of $t_{A}^{f}$ is evaluation of that interpreted term.

Theorem 7.11 states that the standard interpreted solution $e_{A}^{\ddagger}$ of $e$ in the algebra $A$ can be obtained by taking the least fixed point of $R$; in other words the standard interpreted solution $e_{A}^{\ddagger}$ gives the usual denotational semantics.

(ii) Apply the previous example to the RPS of Example 6.4(i). Then Theorem 7.11 states that the standard interpreted solution of the RPS (1.3) in the Elgot algebra $\mathbb{N}_{\perp}$ is obtained as the least fixed point of the function $R$ of Example 7.9. That is, the standard interpreted solution gives the desired factorial function.

(iii) Recall the guarded RPS $e: V \longrightarrow T^{H+V}$ from Example 6.4(ii) whose uninterpreted solution we have described in Example 6.6(ii). Consider again the algebra $\mathbb{N}_{\perp}$ together with the following two operations:

$F_{\mathbb{N}_{\perp}}(x, y, z)=\left\{\begin{array}{ll}\perp & \text { if } x=\perp \text { or } y=\perp \text { or } z=\perp, \\ x & \text { if } x=y \neq \perp, \text { and } z \neq \perp, \\ z & \text { else, }\end{array} \quad G_{\mathbb{N}_{\perp}}(x)= \begin{cases}\left\lfloor\frac{x}{2}\right\rfloor & \text { if } x \in \mathbb{N}, \\ \perp & x=\perp .\end{cases}\right.$

Since the first operation obviously satisfies $F_{\mathbb{N}_{\perp}}(x, y, z)=F_{\mathbb{N}_{\perp}}(y, x, z)$ we have defined an $H$-algebra. It is not difficult to check that the set functor $H$ has a locally continuous lifting $H^{\prime}$ to CPO and that $\mathbb{N}_{\perp}$ is a continuous $H^{\prime}$-algebra. In fact, the existence of the lifting $H^{\prime}$ follows from the fact that the unordered pair functor $V$ : Set $\longrightarrow$ Set can be lifted to CPO; the lifting assigns to a cpo $(X, \leq)$ the set of unordered pairs with the following order: $\{x, y\} \sqsubseteq\left\{x^{\prime}, y^{\prime}\right\}$ iff either $x \leq x^{\prime}$ and $y \leq y^{\prime}$, or $x \leq y^{\prime}$ and $y \leq x^{\prime}$. Thus, we have defined an Elgot algebra for $H$ : Set $\longrightarrow$ Set, see Example 3.10(ii). The standard interpreted solution $e_{\mathbb{N}_{\perp}}^{\ddagger}: V \mathbb{N}_{\perp} \longrightarrow \mathbb{N}_{\perp}$ is given by one commutative binary operation $\varphi_{\mathbb{N}_{\perp}}$ on $\mathbb{N}_{\perp}$. We leave it to the reader to verify that for natural numbers $n$ and $m, \varphi_{\mathbb{N}_{\perp}}(n, m)$ is the natural number represented by the greatest common prefix in the binary representation of $n$ and $m$, e. g., $\varphi_{\mathbb{N}_{\perp}}(12,13)=6$. Notice that we do not have to prove separately that $\varphi_{\mathbb{N}_{\perp}}$ is commutative. In Example 6.4(ii) we have encoded that extra property directly into the RPS $e$ so that any solution must be commutative.

(iv) Least fixed points are RPS solutions. Let $A$ be a poset with joins of all subsets which are at most countable, and let $f: A \longrightarrow A$ be a function preserving joins of ascending chains. Take $f$ and binary joins to obtain an algebra structure on $A$ for the signature functor $H_{\Sigma} X=X+X \times X$ expressing a binary operation symbol $F$ and a unary one $G$. Obviously, this functor has a lifting $H^{\prime}: \mathrm{CPO} \longrightarrow \mathrm{CPO}$ and $A$ is a CPO-enrichable algebra. So $A$ is an Elgot algebra. Turn the formal equations from (1.1) into a recursive program scheme $e: H_{\Phi} \longrightarrow T^{H_{\Sigma}+H_{\Phi}}$ as demonstrated in Section 2.3. The RPS $e$ has a lifting $e^{\prime}: V^{\prime} \longrightarrow T^{H^{\prime}+V^{\prime}}$, where $V^{\prime}$ denotes the lifting of $H_{\Phi}$. The standard interpreted solution $e_{A}^{\ddagger}: V^{\prime} A \longrightarrow A$ gives two continuous functions $\varphi_{A}$ and $\psi_{A}$ on $A$. Clearly, we have $\varphi_{A}(a)=\bigvee_{n \in \mathbb{N}} f^{n}(a)$, and in particular $\varphi_{A}(\perp)$ is the least fixed point of $f$.

7.3. Interpreted Solutions in CMS. Recall the category CMS of complete metric spaces from Example 2.4. and also the facts that the contracting endofunctors are iteratable and closed under finite coproducts. Let 
$H, V:$ CMS $\longrightarrow$ CMS be such contracting endofunctors. We shall show in this subsection that for any guarded RPS $e: V \longrightarrow T^{H+V}$, we can find a unique interpreted solution in any non-empty $H$-algebra $A$. To this end, assume that we have such a guarded RPS $e$, and let $(A, a)$ be a non-empty $H$-algebra. Then $A$ is a cia, and, in particular it carries the structure of an Elgot algebra. Notice that for any non-expanding map $t: V A \longrightarrow A$ we obtain an algebra structure $[a, t]:(H+V) A \longrightarrow A$. This turns $A$ into a cia for $H+V$, and thus we have the evaluation morphism

$$
\widetilde{[a, t]}: T^{H+V} A \longrightarrow A
$$

As in CPO, the RPS $e$ induces a function $R$ on $\operatorname{CMS}(V A, A)$, see (7.13). The standard procedure for obtaining an interpreted solution would be to recall that $\operatorname{CMS}(V A, A)$ is a complete metric space and then to prove that $R$ is a contracting map on it. We then invoke Banach's Fixed Point theorem to obtain a unique fixed point of $R$. Here we simply apply Theorem 7.5. Notice, however, that we cannot completely avoid Banach's Fixed Point theorem: it is used in the proof that final coalgebras exist for contracting functors, see [ARe].

Theorem 7.15. The unique interpreted solution $e_{A}^{\ddagger}: V A \longrightarrow A$ of $e$ in $A$ as obtained in Theorem 7.5 is the unique fixed point of the function $R$ on $\operatorname{CMS}(V A, A)$ defined by 7.13).

Proof. We will prove that fixed points of $R$ are in one-to-one correspondence to interpreted solutions. In fact, any interpreted solution $e_{A}^{\ddagger}: V A \longrightarrow A$ is a fixed point of $R$ by Lemma 7.3 (see (7.4)). Conversely, we will now prove that any fixed point $t$ of $R$ is an interpreted solution of $e$ in $A$. Let $t$ be a fixed point of $R$ and write $\beta=\widetilde{[a, t]}$. We are finished if we prove the commutativity of (7.1). We write $T=T^{H+V}$ and $\lambda=\left[\kappa_{A}^{H+V} \varnothing \mathrm{inl}, e_{A}\right]$ for short once again. Recall that $T A$ is the free cia on $A$ and that $\beta: T A \longrightarrow A$ is the unique homomorphism of Elgot algebras from $T A$ to $A$ extending $i d_{A}$, i. e. such that the equation $\beta \varnothing \eta_{A}^{H+V}=i d_{A}$ holds. From Example 3.3(iv) we see that $(A,[a, t])$ is a cia for $H+V$. Thus, by Proposition 3.7 $\beta$ is the unique homomorphism of algebras for $H+V$ extending $i d_{A}$. So in order to verify the desired equation $\beta \varnothing \widehat{e}_{A}=\beta$ we verify below that the following diagram commutes:

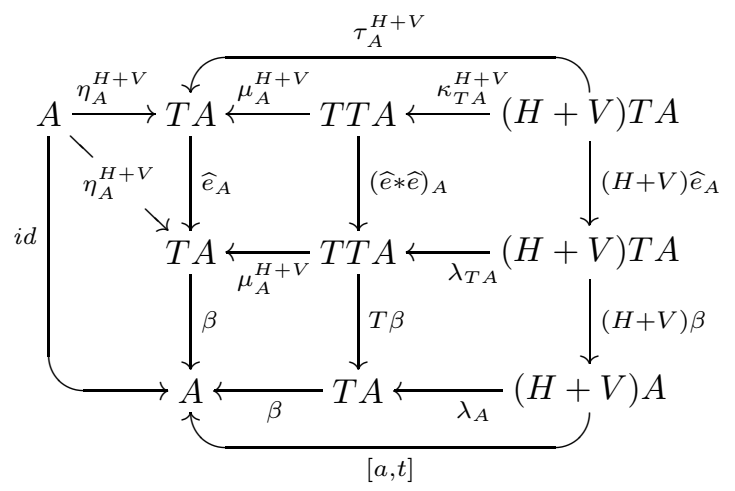

In fact, the upper part commutes by Corrollary 3.17 the upper triangle and the upper left-hand square commute since $\widehat{e}$ is a monad morphism; the upper right-hand square commutes by the double interchange law and the definition of $\widehat{e}$ (see Lemma 6.9); the lower triangle commutes and the lower left-hand square both commute since the evaluation morphism $\beta$ is an Eilenberg-Moore algebra for the monad $T$; the lower right-hand square commutes by the naturality of $\lambda$. Finally, we verify the commutativity of the lowest part componentwise: the left-hand component commutes by Equation (7.3), and the right-hand part $\beta \varnothing e_{A}=t$ holds since $t$ is a fixed point of $R$.

Remark 7.16. Let $H_{\Sigma}$ be a signature functor on Set and denote by $H^{\prime}$ a lifting to CMS as described in Example 3.3 (vii). For a complete metric space $Y$ the final coalgebra $T^{H^{\prime}} Y$ for $H^{\prime}\left({ }_{-}\right)+Y$ is the set $T_{\Sigma} Y$ of all $\Sigma$-trees over $Y$ equipped with a suitable complete metric. This metric can be described as follows. Recall from ARe that $T^{H^{\prime}} Y$ is obtained as $T_{\omega}$ after $\omega$ steps of the final coalgebra chain for $H^{\prime}\left({ }_{-}\right)+Y$ (see Construction 2.5). That means the metric on $T_{\Sigma} Y$ is the smallest metric such that all projections $t_{\omega, i}: T_{\Sigma} Y=T_{\omega} \longrightarrow T_{i}$ are non-expanding. We illustrate this with an example adapted from [ARe]. Let $H_{\Sigma} X=X \times X$ be the functor expressing one binary operation symbol $*$. Then we can represent $T_{0}=1$ by a single node tree labelled with $\perp$ and $T_{i+1}=T_{i} \times T_{i}+Y$ by trees which are either single node trees labelled 
in $Y$, or which are composed by joining two trees from $T_{i}$ with a root labelled by $*$ :

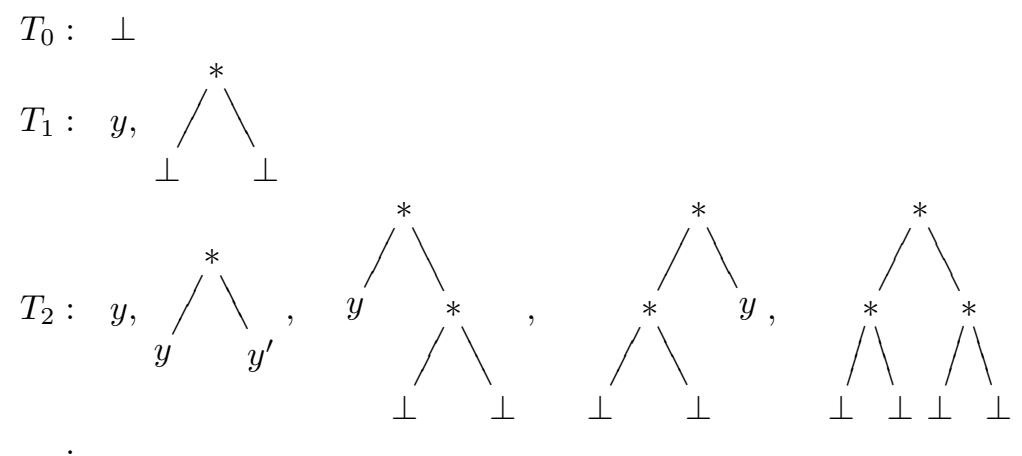

The distance on $T_{1}$ is that of $Y$ for single node trees and 1 otherwise. The distance on $T_{2}$ is again that of $Y$ between single node trees, and 1 between single node trees and all other trees. Furthermore, the distance between trees of different shapes is $\frac{1}{2}$, and finally, $d_{T_{2}}\left(y * y^{\prime}, z * z^{\prime}\right)=\frac{1}{2} \max \left\{d_{Y}(y, z), d_{Y}\left(y^{\prime}, z^{\prime}\right)\right\}$ as well as $d_{T_{2}}\left(y * t, y^{\prime} * t\right)=d_{T_{2}}\left(t * y, t * y^{\prime}\right)=\frac{1}{2} d_{Y}\left(y, y^{\prime}\right)$, where $t=\perp * \perp$, etc. In general, the distance on $T_{i+1}$ is that of $Y$ between single node trees, it is 1 between single node trees and trees of height at least 1, and otherwise we have $d_{T_{i+1}}\left(s * t, s^{\prime} * t^{\prime}\right)=\frac{1}{2} \max \left\{d_{T_{i}}\left(s, s^{\prime}\right), d_{T_{i}}\left(t, t^{\prime}\right)\right\}$. For the metric on $T_{\Sigma} Y$, we have

$$
d_{T_{\Sigma} Y}\left(s_{1}, s_{2}\right)=\sup _{i<\omega} d_{T_{i}}\left(t_{\omega, i}\left(s_{1}\right), t_{\omega, i}\left(s_{2}\right)\right) .
$$

This is the smallest metric for which the projections are non-expanding. (One may also verify directly that this definition gives a complete metric space structure and that $H^{\prime}\left(_{-}\right)+Y$ preserves the limit, so that we indeed have a final coalgebra.) Finally notice that the metric of $T_{\Sigma} Y$ depends on the choice of the lifting $H^{\prime}$. For example, if we lift the functor $H_{\Sigma}$ as $H^{\prime}(X, d)=\left(X^{2}, \frac{1}{3} d_{\max }\right)$, the factor $\frac{1}{2}$ would have to be replaced by $\frac{1}{3}$ systematically.

\section{Example 7.17.}

(i) Consider the endofunctor $H^{\prime}: \mathrm{CMS} \longrightarrow \mathrm{CMS}$ obtained by lifting the signature functor $H_{\Sigma} X=$ $X \times X+X$ expressing a binary operation $F$ and a unary one $G$ as described in Example 2.9. We use a contraction factor $\varepsilon=\frac{1}{2}$. The Euclidean interval $I=[0,1]$ together with the operations $F(x, y)=\frac{x+y}{4}$ and $G(x)=\frac{\sin (x)}{2}$ is an $H^{\prime}$-algebra, whence a cia. Use only the first equation in (1.1) to obtain a guarded RPS $e: I d \longrightarrow T^{H_{\Sigma}+I d}$ where $I d$ expresses the unary operation symbol $\varphi$. Let $V^{\prime}$ be contracting lifting of $I d$, again with a contraction factor of $\varepsilon=\frac{1}{2}$. Then $e$ gives rise to a guarded RPS $e^{\prime}: V^{\prime} \longrightarrow T^{H^{\prime}+V^{\prime}}$ in CMS. The unique interpreted solution of $e^{\prime}$ in $I$ consists of a function $\varphi_{I}: I \longrightarrow I$ satisfying $\varphi_{I}(x)=\frac{1}{4}\left(x+\varphi_{I}\left(\frac{1}{2} \sin x\right)\right)$, that is, $\varphi_{I}$ is the unique function $f$ satisfying (1.4). Please note that at long last the theory of recursive program schemes has provided new results: it is not obvious that there is any $\varphi_{I}$ satisfying this equation.

(ii) Self-similar sets are solutions of interpreted program schemes. Recall from Example 3.3. (v) that for any complete metric space $(X, d)$, we obtain the complete metric space $(C(X), h)$ of all non-empty compact subspaces of $X$ with the Hausdorff metric. Furthermore, contractive mappings of $X$ yield structures of cias on $C(X)$. Now consider the functor $H^{\prime}$ on CMS with $H^{\prime}(X, d)=\left(X^{3}, \frac{1}{3} d_{\max }\right)$, where $d_{\max }$ is the maximum metric. It is a lifting of the signature functor $H_{\Sigma}$ on Set expressing one ternary operation $\alpha$. Let $A=[0,1] \times[0,1]$, be equipped with the usual Euclidean metric. Consider the contracting maps $f(x, y)=\left(\frac{1}{3} x, \frac{1}{3} y\right), g(x, y)=\left(\frac{1}{3} x+\frac{1}{3}, \frac{1}{3} y\right)$, and $h(x, y)=\left(\frac{1}{3} x+\frac{2}{3}, \frac{1}{3} y\right)$ of $A$. Then it follows that $\alpha_{A}: C(A)^{3} \longrightarrow C(A)$ with $\alpha(D, E, F)=f[D] \cup g[E] \cup h[F]$ is a $\frac{1}{3}$-contracting map, whence a structure of a cia for $H^{\prime}$. The formal equation

$$
\varphi(x) \approx \alpha(\varphi(x), x, \varphi(x))
$$

gives rise to a guarded RPS $e: I d \longrightarrow T^{H_{\Sigma}+I d}$, where the identity functor expresses the operation $\varphi$. If we take the lifting of $I d$ to CMS which is given by $V^{\prime}(X, d)=\left(X, \frac{1}{3} d\right)$, then $e$ gives rise to a natural transformation $e^{\prime}: V^{\prime} \longrightarrow T^{H^{\prime}+V^{\prime}}$. Its interpreted solution in the cia $C(A)$ is a $\frac{1}{3}$-contracting map $\varphi_{A}: C(A) \longrightarrow C(A)$ which maps a non-empty compact subspace $U$ of $A$ to a space of the following form: $\varphi_{A}(U)$ has three parts, the middle one is a copy of $U$ scaled by $\frac{1}{3}$, and the left-hand and right-hand one look like copies of the whole space $\varphi_{A}(U)$ scaled by $\frac{1}{3}$. For example, we have the 
assignment
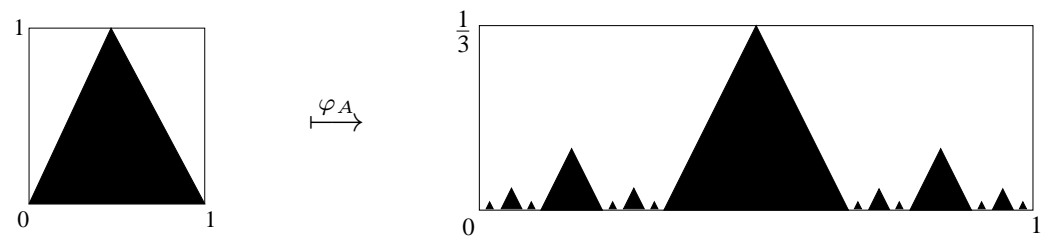

(iii) Coming back to Example [3.3(vi), let us consider $\left(C(I), \alpha_{I}\right)$, where $I=[0,1]$ is the Euclidean interval, $C(I)$ is the set of all non-empty closed subsets of $I$, and $\alpha_{I}$ is the structure of a cia arising from $f(x)=\frac{1}{3} x$ and $g(x)=\frac{1}{3} x+\frac{2}{3}$ as described is Example 3.3. $(\mathrm{v})$. The formal equation

$$
\varphi(x) \approx \alpha(\varphi(x), x)
$$

gives similarly as in (i) above a guarded RPS $e: I d \longrightarrow T^{H_{\Sigma}+I d}$, where $H_{\Sigma} X=X \times X$ now expresses the binary operation $\alpha$. Again, we have liftings $V^{\prime}(X, d)=\left(X, \frac{1}{3} d\right)$ and $H^{\prime}(X, d)=\left(X^{2}, \frac{1}{3} d_{\max }\right)$ of $I d$ and $H_{\Sigma}$, respectively. So the RPS $e$ lifts to the guarded RPS $e^{\prime}: V^{\prime} \longrightarrow T^{H^{\prime}+V^{\prime}}$ in CMS. Its unique interpreted solution is given by the $\frac{1}{3}$-contracting map $\varphi_{I}: C(I) \longrightarrow C(I)$ satisfying $\varphi_{I}(t)=\alpha_{I}\left(\varphi_{I}(t), t\right)=f\left[\varphi_{I}(t)\right] \cup g[t]$ for every non-empty closed subset $t$ of the interval $I$.

\section{Conclusions and Future Work}

We have presented a general and conceptually clear way of treating the uninterpreted and the interpreted semantics of recursive program schemes in a category theoretic setting. For this we have used recent results on complete Elgot algebras and results from the theory of coalgebras. We have shown that our theory readily specializes to the classical setting yielding denotational semantics using complete partial orders or complete metric spaces. We have presented new applications of recursive program scheme solutions including fractal self-similarity and also applications which cannot be handled by the classical methods; defining operations satisfying equations like commutativity. Another new application, recursively defined functions on nonwellfounded sets, will be treated in a future paper.

Now one must go forward in reinventing algebraic semantics with category theoretic methods. We strongly suspect that there is much to be said about the relation of our work to operational semantics. We have not investigated higher-order recursive program schemes using our tools, and it would be good to know whether our approach applies in that area as well. The paper [MU] addresses variable binding and infinite terms coalgebraically, and this may well be relevant. Back to the classical theory, one of the main goals of the original theory is to serve as a foundation for program equivalence. It is not difficult to prove the soundness of fold/unfold transformations in an algebraic way using our semantics; this was done in $\mathrm{Mo}_{2}$ for uninterpreted schemes. We study the equational properties of our very general formulation of recursion in $\mathrm{MM}_{2}$. One would like more results of this type. The equivalence of interpreted schemes in the natural numbers is undecidable, and so one naturally wants to study the equivalence of interpreted schemes in classes of interpretations. The classical theory proposes classes of interpretations, many of which are defined on ordered algebras, see $\mathrm{G}$. It would be good to revisit this part of the classical theory to see whether Elgot algebras suggest tractable classes of interpretations.

Another path of future research is the study of algebraic trees with categorical methods. In the setting of trees over a signature $\Sigma$ the solutions of recursive program schemes form the theory of algebraic trees, a subtheory of the theory of all trees on $\Sigma$. Moreover, algebraic trees are closed under second-order substitution and they form an iterative theory in the sense of Elgot [E. Similar results should be possible to obtain in our generalized categorical setting.

\section{ACKNOWLEDGMENTS}

We are grateful to Jiří Adámek for many conversations on this and related matters, and for his enthusiasm for our project. We would also like to thank the anonymous referees for their suggestions to improve this text. 


\section{Notations}

$T^{H} X$

$\alpha_{X}^{H}$

$\tau_{X}^{H}$
$\eta_{X}^{H}$

$\mu^{H}$

$\kappa^{H}$

$\Sigma$

$H_{\Sigma}$

$(f, \vec{x})$

$T_{\Sigma} X$

Set

$\mathrm{CPO}$

CMS

$T_{\lambda}$

$\mathcal{P}_{\mathrm{f}}$

$H^{\prime}$

$\alpha * \beta$

$\operatorname{Mon}(\mathcal{A})$

$J$

$[\mathcal{A}, \mathcal{B}]$

$f(\vec{x}) \approx t^{f}(\vec{x})$

$e: V \longrightarrow T^{H+V}$

$e: X \longrightarrow H X+A$

$e: X \longrightarrow S(X+Y)$

$e^{\dagger}$

$C(X)$

$h \bullet e$

$f=e$

$\left(A, a,(\mathrm{z})^{\dagger}\right)$

$p(x) \downarrow(p(x) \uparrow)$

$p(x) \simeq q(y)$

$\operatorname{Alg}^{\dagger} H$

$\widetilde{a}: T A \longrightarrow A$

$\left(S, \eta, \mu, S^{\prime}, \iota, \mu^{\prime}\right)$

$\sigma: H \longrightarrow S$ a final coalgebra for $H\left({ }_{-}\right)+X$, p. 6] a free cia or a free Elgot algebra on $X$, Theorem 3.14 object map of a free completely iterative monad on $H$, Theorems 3.15 and 4.6 .

$\alpha_{X}^{H}=\left[\tau_{X}^{H}, \eta_{X}^{H}\right]^{-1}: T^{H} X \cong H T^{H} X+X$, a final coalgebra, p. 6] and Theorems 3.14 and 3.15 .

$H$-algebra structure of the free cia $T^{H} X$, p. 6 and Theorem 3.14 .

universal arrow of the free cia $T^{H} X, \mathrm{p} .6$ and Theorems 3.14 and 3.15, unit of the free completely iterative monad $T^{H}$, Theorem 4.6.

$\mu^{H}: T^{H} T^{H} \longrightarrow T^{H}$ is the multiplication of the the free completely iterative monad $T^{H}$, Theorem 3.15(1b), p. 19, and Theorem 4.6. p. 23.

$\kappa^{H}: H \longrightarrow T^{H}$ is the universal arrow of the free completely iterative monad $T^{H}$, Theorem 3.15 (4), p. 19, and Theorem 4.6, p. 23

a signature of function symbols, also regarded as a functor $\Sigma: \mathbb{N} \longrightarrow$ Set, Example 2.1. p. 6.

a signature functor, see (2.1), p. 6.

generic element of $H_{\Sigma} X, f \in \Sigma_{n}, x \in X^{n}$, p. 6 .

all $\Sigma$-trees over $X, \mathrm{p}$. 6 .

the category of sets and maps, p. 6f.

the category of complete partial orders (not necessarily with a least element) and continuous maps, Example 2.3. p. 7 .

the category of complete metric spaces with distances in $[0,1]$ and non-expanding maps, Example 2.4 p. 2.4.

the $\lambda$ th step in the final coalgebra construction, Construction 2.5] p. 7.

finitary powerset functor $X \longmapsto\{A \mid A \subseteq X$ finite $\}$, Example 2.7(ii), p. 8.

lifting of a set endofunctor $H$ to CPO or CMS, respectively, Examples 2.8, p. 8, and 2.9, p. 9.

parallel composition of natural transformations $\alpha$ and $\beta$, p. 10 .

the category of monads and their homomorphisms on the category $\mathcal{A}, \mathrm{p} .10$.

the canonical inclusion $J: \mathbb{N} \longrightarrow$ Set, $n \longmapsto\{0, \ldots, n-1\}$, p. 10 .

the category of endofunctors from $\mathcal{A}$ to $\mathcal{B}$ and natural transformations between them, p. 10.

classical form of a recursive program scheme as system of mutually recursive formal function definitions, (2.5), p. 11.

a recursive program scheme, p. 11 and Definition 6.1. p. 30.

a flat equation morphism, Definition 3.1. p. 13.

an equation morphism w.r.t. the ideal monad $S$, Definition 4.5, p. 22 ,

a solution of a (flat) equation morphism $e$ or of a recursive program scheme $e,(3.2)$, p. 13, p. 23, and (6.2), p. 30, respectively.

the non-empty compact subspaces of a complete metric space $X$, Example $[3.3$ (v), p. 14.

the "renaming of parameters" of a flat equation morphism $e: X \longrightarrow H X+A$ by a morphism $h: A \longrightarrow B$, Remark 3.4, p. 15.

"simultaneous" flat equation morphism obtained from $e: X \longrightarrow H X+Y$ and $f: Y \longrightarrow H Y+A$, Remark 3.4. p. 15.

a (complete) Elgot algebra, Definition 3.5 , p. 15.

partial function $p$ is (not) defined, p. 17

Kleene equality: $p(x)$ is defined iff $q(y)$ is defined and if both are defined, they are equal, p. 15

the category of Elgot algebras and their homomorphisms, Theorem 3.15, p. 19.

the evaluation morphism of an Elgot algebra $\left(A, a,\left(_{-}\right)^{\dagger}\right),(3.6)$, p. 19, and Theorem 3.16, p. 20.

an ideal monad, Definition 4.3, p. 22

an ideal natural transformation from the functor $H$ to the ideal monad $S, \sigma=$

$H \stackrel{\sigma^{\prime}}{\longrightarrow} S^{\prime} \stackrel{\iota}{\longrightarrow} S$ for some $\sigma^{\prime}$, Definition 4.3 p. 22 
$\bar{\sigma}$

$H / \operatorname{Mon}(\mathcal{A})$

$H / \operatorname{CIM}(\mathcal{A})$

$\mathcal{H}$

$f: V \longrightarrow H T^{H+V}$

$\underline{t}$

$\bar{e}$

$\widehat{e}$

$e_{A}^{\ddagger}$

$\mathbb{N}_{\uparrow}$

$\mathbb{N}_{\perp}$ unique extension of an ideal natural transformation $\sigma: H \longrightarrow S$ to a monad morphism $\bar{\sigma}: T^{H} \longrightarrow S$, Theorem 4.6. p. 23.

the subcategory of the comma-category $H /[\mathcal{A}, \mathcal{A}]$ formed by natural transformation from $H$ to monads $S$ and monad morphisms, p. 25.

the subcategory of $H / \operatorname{Mon}(\mathcal{A})$ given by ideal natural transformations from $H$ into completely iterative monads $S$ and ideal monad morphisms, p. 25

the endofunctor on $H / \operatorname{Mon}(\mathcal{A})$ and $H / \operatorname{CIM}(\mathcal{A})$, respectively, given by $(S, \sigma) \longmapsto$ $(H S+I d$, inl $\varnothing H \eta)$, p. 25, Lemma 5.2, p. 25, Corollary 5.5. p. 29.

a natural transformation exhibiting a recursive program scheme $e: V \longrightarrow T^{H+V}$ as guarded, Definition 6.1, p. 30.

a formal variable arising from a recursive program scheme. Here $t$ is a tree on $\Sigma+\Phi$ allowing some additional variables as well, (1.7), p. 4. Remark 6.10, p. 33.

the ideal monad morphism $\bar{e}: T^{H+V} \longrightarrow H T^{H+V}+I d$ is induced by the guarded recursive program scheme $e$, and it yields a coalgebra for the endofunctor $\mathcal{H}$ of $H / \operatorname{CIM}(\mathcal{A})$, Lemma 6.9. p. 33 .

the ideal monad (endo)morphism $\widehat{e}: T^{H+V} \longrightarrow T^{H+V}$ is induced by the guarded recursive program scheme $e$, Lemma 6.9, p. 33.

an interpreted solution of a recursive program scheme $e: V \longrightarrow T^{H+V}$ in an Elgot algebra $\left(A, a,\left(_{-}\right)^{*}\right)$, Definition 7.1 , p. 37.

the natural numbers with the computation tree Elgot algebra structure, p. 41 and Proposition 3.12 p. 17

the flat cpo obtained from the natural numbers $\mathbb{N}$ by adding a least element $\perp$, Example 7.9. p. 43. 


\section{REFERENCES}

[A] Peter Aczel, Non-Well-Founded Sets. CSLI Lecture Notes Number 14, CSLI Publications, Stanford, 1988.

[AAV] P. Aczel, J. Adámek and J. Velebil, A Coalgebraic View of Infinite Trees and Iteration, Electron. Notes Theor. Comput. Sci. 44 (2001), no. 1, 26 pp.

[AAMV] P. Aczel, J. Adámek, S. Milius and J. Velebil, Infinite Trees and Completely Iterative Theories: A Coalgebraic View, Theoret. Comput. Sci. 300 (2003), 1-45.

$[\mathrm{ABM}] \quad$ J. Adámek, S. L. Bloom and S. Milius, On algebras with Iteration, preprint, 2006.

[AK] J. Adámek and V. Koubek, On the greatest fixed point of a set functor, Theoret. Comput. Sci. 150 (1995), 57-75.

[AM] J. Adámek and S. Milius, Terminal Coalgebras and Free Iterative Theories, Inform. and Comput. 204 (2006), 11391172 .

$\left[\mathrm{AMV}_{1}\right] \quad$ J. Adámek, S. Milius and J. Velebil, Free Iterative Theories: A Coalgebraic View, Math. Structures Comput. Sci. 13 (2003), 259-320.

$\left[\mathrm{AMV}_{2}\right]$ J. Adámek, S. Milius and J. Velebil, Iterative Algebras at Work, accepted for publication in Math. Structures Comput. Sci., available at the URL http://www.stefan-milius.eu, extended abstract appeared in Electron. Notes Theor. Comput. Sci. 106 (2004), 3-24.

$\left[\mathrm{AMV}_{3}\right] \quad$ J. Adámek, S. Milius and J. Velebil, On coalgebra based on classes, Theoret. Comput. Sci. 316 (2004), 3-23.

$\left[\mathrm{AMV}_{3}\right] \quad$ J. Adámek, S. Milius and J. Velebil, Elgot Algebras, Logical Methods in Computer Science Vol. 2 (5:4), 31 pp.

[AP] J. Adámek and H.-E. Porst, On tree coalgebras and coalgebra presentations, Theoret. Comput. Sci. 311 (2004), $257-283$.

[ARe] J. Adámek and J. Reitermann, Banach's Fixed-Point Theorem as a Base for Data-Type Equations, Appl. Categ. Structures 2 (1994), 77-90.

[AT] J. Adámek and V. Trnková, Automata and Algebras in Categories. Kluwer Academic Publishers, 1990.

[ARu $] \quad$ P. America and J. J. M. M. Rutten, Solving Reflexive Domain Equations in a Category of Complete Metric Spaces, J. Comput. System Sci. 39 (1989), 343-375.

[AN] A. Arnold and M. Nivat, The metric space of infinite trees. Algebraic and topological properties, Fund. Inform. III, no. 4 (1980), 445-476.

[B] M. F. Barnsley, Fractals Everywhere, Academic Press 1988.

[Ba] M. Barr, Terminal coalgebras in well-founded set theory, Theoret. Comput. Sci. 114 (1993), 299-315.

[BM] J. Barwise and L. S. Moss, Vicious Circles, CSLI Publications, Stanford, 1996.

[Bl] S. L. Bloom, All Solutions of a System of Recursion Equations in Infinite Trees and Other Contraction Theories, J. Comput. System Sci. 27 (1983), 225-255.

[BE] S. L. Bloom and Z. Ésik, Iteration Theories: The equational logic of iterative processes, EATCS Monographs on Theoretical Computer Science, Berlin: Springer-Verlag (1993).

[Bo] F. Borceux, Handbook of Categorical Algebra 2: Categories and Structures, Vol. 51 of Encyclopedia of Mathematics and its Applications, Cambridge University Press, 1994.

[C] B. Courcelle, Fundamental properties of infinite trees, Theoret. Comput. Sci. 25 (1983), no. 2, 95-169.

[E] C. C. Elgot, Monadic Computation and Iterative Algebraic Theories, in: Logic Colloquium '73 (eds: H. E. Rose and J. C. Shepherdson), North-Holland Publishers, Amsterdam, 1975.

[EBT] C. C. Elgot, S. L. Bloom and R. Tindell, On the Algebraic Structure of Rooted Trees, J. Comput. System Sci. 16 (1978), 361-399.

[GLMP 1$]$ N. Ghani, C. Lüth, F. De Marchi and A. J. Power, Algebras, coalgebras, monads and comonads, Electron. Notes Theor. Comput. Sci. 44 (2001), no. 1, 18 pp.

$\left[\mathrm{GLMP}_{2}\right]$ N. Ghani, C. Lüth, F. De Marchi and A. J. Power, Dualising initial algebras, Math. Structures Comput. Sci. 13 (2003), no. 2, 349-370.

[GLM] N. Ghani, C. Lüth and F. De Marchi, Solving Algebraic Equations using Coalgebra, Theor. Inform. Appl. 37 (2003), 301-314.

[G] I. Guessarian, Algebraic Semantics. Lecture Notes in Comput. Sci., Vol. 99, Springer, 1981.

[L] J. Lambek, A Fixpoint Theorem for Complete Categories, Math. Z. 103 (1968), 151-161.

$\left[\mathrm{L}_{1}\right] \quad$ T. Leinster, General self-similarity: an overview, e-print math.DS/0411343 v1.

$\left[\mathrm{L}_{2}\right] \quad$ T. Leinster, A general theory of self-similarity I, e-print math.DS/041344.

$\left[\mathrm{L}_{3}\right] \quad$ T. Leinster, A general theory of self-similarity II, e-print math.DS/0411345

[ML] S. Mac Lane, Categories for the working mathematician, 2nd edition, Springer Verlag, 1998.

[MU] R. Matthes and T. Uustalu, Substitution in Non-Wellfounded Syntax with Variable Binding. In H. P. Gumm, (ed.), Electron. Notes Theor. Comput. Sci., 82 (2003), no. 1, 15 pp.

$\left[\mathrm{Mi}_{1}\right] \quad$ S. Milius, On Iteratable Endofunctors, Electron. Notes Theor. Comput. Sci. 69 (2002), 18 pp.

$\left[\mathrm{Mi}_{2}\right] \quad$ S. Milius, Completely Iterative Algebras and Completely Iterative Monads, Inform. and Comput. 196 (2005), 1-41.

$[\mathrm{MM}] \quad$ S. Milius and L. S. Moss, The Category Theoretic Solution of Recursive Program Schemes, extended abstract, in Proceedings of the 1st International Conference on Algebra and Coalgebra in Computer Science (CALCO 2005), Lecture Notes in Comput. Sci. 3629 (2005), 293-312.

$\left[\mathrm{MM}_{2}\right] \quad$ S. Milius and L. S. Moss, Equational Properties of Solutions to Recursive Program Schemes, working draft.

$\left[\mathrm{MM}_{3}\right] \quad$ S. Milius and L. S. Moss, Recursive Program Scheme Solutions in Hypersets, working draft.

[Mo1] L. S. Moss, Parametric Corecursion, Theoret. Comput. Sci. 260 (2001), no. 1-2, 139-163.

$\left[\mathrm{Mo}_{2}\right] \quad$ L. S. Moss, The Coalgebraic Treatment of Second-Order Substitution and Uninterpreted Recursive Program Schemes, preprint, 2002.

[Mon $]$ L. S. Moss, Uniform Functors on Sets, in K. Futatsugi et al. (eds.), Algebra, Meaning, and Computation: A Festschrift in Honor of Prof. Joseph Goguen, Lecture Notes Comput. Sci. 4064 (2006), Springer-Verlag, Berlin, 420-448. 
[N] M. Nivat, On the Interpretation of Recursive Polyadic Program Schemes, Symposia Mathematica XV (1975), 255281.

[W] J. Worrell, On the Final Sequence of a Finitary Set Functor, Theoret. Comput. Sci. 338 (2005), 184-199.

Institute of Theoretical Computer Science, Technical University of Braunschweig, Germany

E-mail address: milius@iti.cs.tu-bs.de

Department of Mathematics, Indiana University, Bloomington, IN, USA

E-mail address: 1sm@cs.indiana.edu 